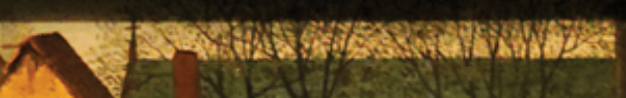

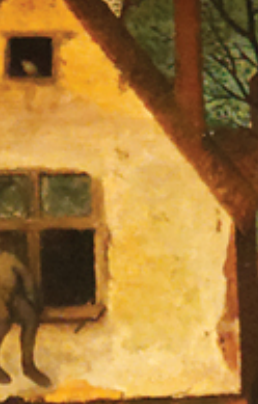

2.

(4)

t.)

w

o. i) 2 .

4

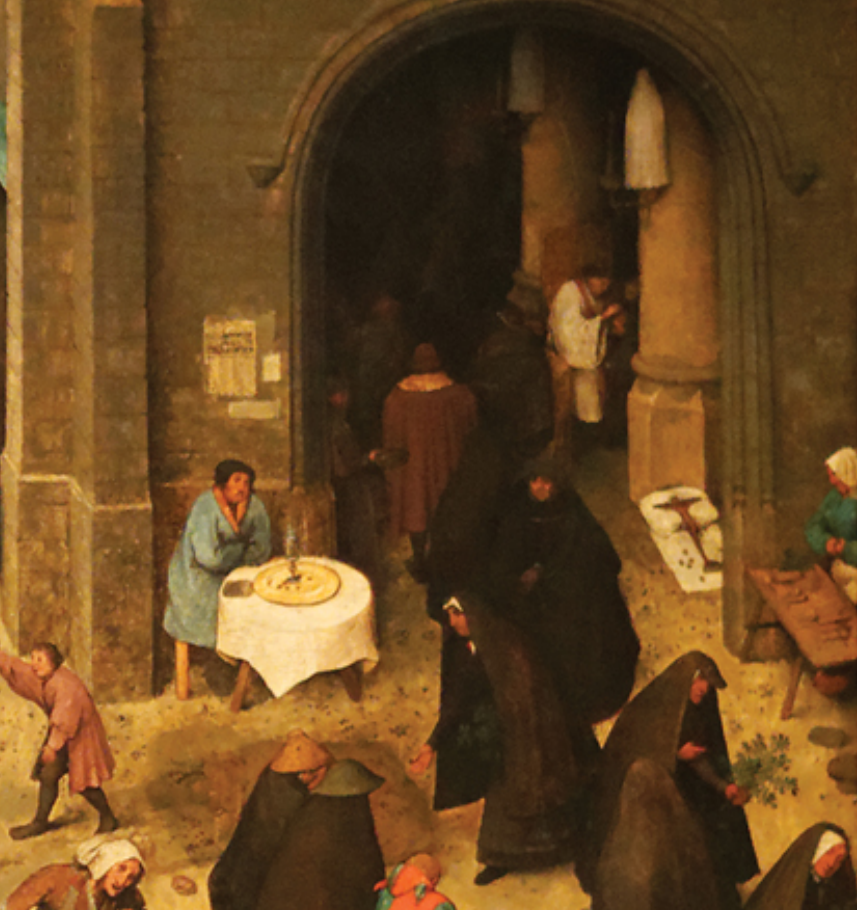

Multiagent

Industrial

Symbiosis

System s

a. is

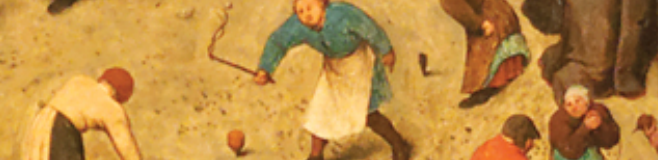

4

$4=14$ ant

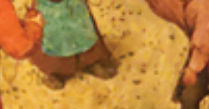

$\frac{1}{8}$
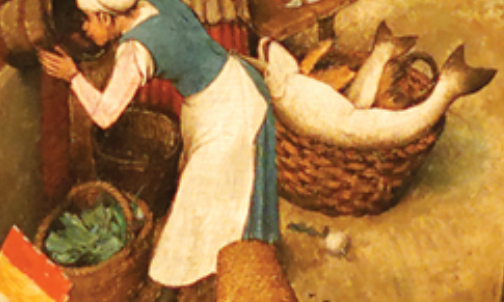

(S)

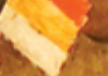

(a)

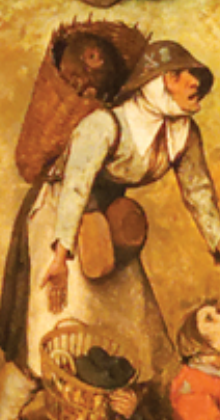

$\cos ^{2}=\frac{1}{(3)} \mathrm{k}$

1)

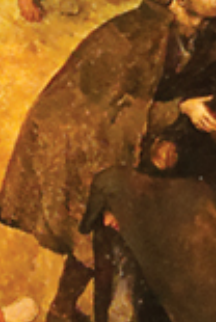

$\frac{1}{6}$

$x$

a)

(i) 271

(3)

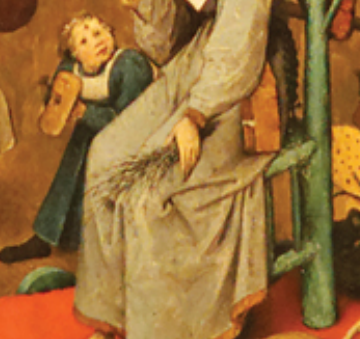

2)

8 ised
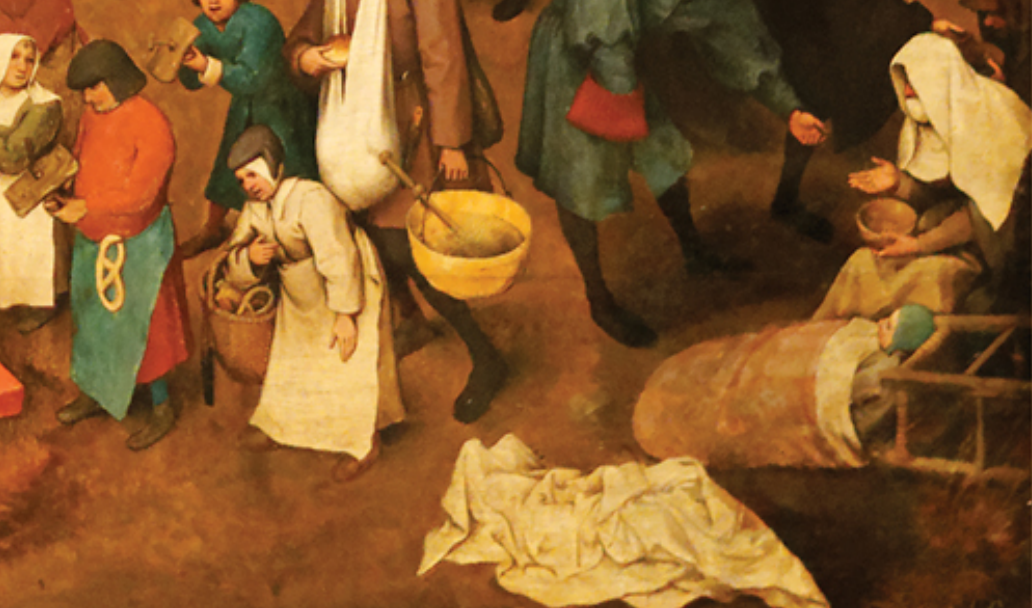

Vahid Yazdanpanah

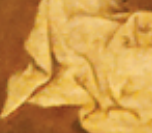





\title{
Multiagent Industrial Symbiosis Systems
}

\author{
VAHID YaZDANPANAH
}





\title{
MULTIAGENT INDUSTRIAL SYMBIOSIS SYSTEMS
}

\section{DISSERTATION}

\author{
TO OBTAIN \\ THE DEGREE OF DOCTOR AT THE UNIVERSITY OF TWENTE, \\ ON THE AUTHORITY OF THE RECTOR MAGNIFICUS, \\ Prof. Dr. T. T. M. Palstra, \\ ON ACCOUNT OF THE DECISION OF THE GRADUATION COMMITTEE, \\ TO BE PUBLICLY DEFENDED \\ ON Wednesday, November 27, 2019, AT I4:45 HOURS
}

BY

\section{VAHID YAZDANPANAH}


This dissertation has been approved by:

the first supervisor,

Prof. dr. W.H.M. Zijm,

the second supervisor,

Prof. dr. J. van Hillegersberg,

and the co-supervisor,

Dr. D.M. Yazan.

\section{UNIVERSITY \\ OF TWENTE.}

Ph.D. dissertation, University of Twente, Enschede, The Netherlands

Department of Industrial Engineering and Business Information Systems

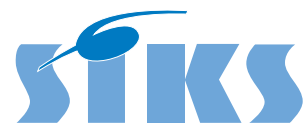

SIKS Dissertation Series No. 2019-30

The research reported in this thesis has been carried out under the auspices of SIKS, the Dutch Research School for Information and Knowledge Systems.

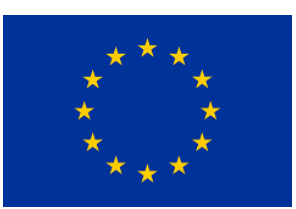

The research contained in this dissertation has been funded by the European Union's Horizon 2020 research and innovation programme under grant agreement No. 680843.

Printed by: Ipskamp Printing, Enschede, The Netherlands

Cover Painting: "The Fight Between Carnival and Lent” by Pieter Bruegel the Elder

Cover Design: V. Yazdanpanah

ISBN: 978-90-365-4887-8

DOI: $10.3990 / 1.9789036548878$

(C)219 - Vahid Yazdanpanah, 2019, Enschede, The Netherlands

All rights reserved. No part of this dissertation may be reproduced, stored in a retrieval system or transmitted in any form or by any means without permission of the author. 
Graduation Committee:

Chairman and Secretary:

First Supervisor:

Second Supervisor:

Co-supervisor:

Members:
Prof. dr. T.A.J. Toonen

University of Twente, The Netherlands

Prof. dr. W.H.M.Zijm

University of Twente, The Netherlands

Prof. dr. J. van Hillegersberg

University of Twente, The Netherlands

Dr. D.M. Yazan

University of Twente, The Netherlands

Prof. dr. V. Albino

Politecnico di Bari, Italy

Prof. dr. M. Dastani

Utrecht University, The Netherlands

Prof. dr. T. Filatova

University of Twente, The Netherlands

Prof. dr. K. Gibert

Polytechnic University of Catalonia, Spain

Dr. M.R.K. Mes

University of Twente, The Netherlands 



\title{
Multiagent Industrial Symbiosis Systems
}

\author{
Vahid Yazdanpanah
}

\begin{abstract}
Multiagent Systems (MAS) research reached a maturity to be confidently applied to reallife complex problems. Successful application of MAS methods for behavior modeling, strategic reasoning, and decentralized governance, encouraged us to focus on applicability of MAS techniques in a class of industrial systems and to develop multiagent models, coordination methods, and decision support tools for this context. We direct attention towards a form of industrial practices called Industrial Symbiosis Systems (ISS) as a highly dynamic domain of application for MAS techniques. In ISS, firms aim to reduce their material and energy footprint by circulating reusable resources among the participants. To enable systematic reasoning about ISS behavior and support firms' (as well as ISS designers') decisions, we saw the opportunity for marrying industrial engineering with multiagent systems research. This enabled the introduction of contextualized representation frameworks to reason about the dynamics of ISS, operational semantics to develop computational models for ISS, coordination mechanisms to enforce desirable ISS implementations, and transaction cost allocation
\end{abstract}


methods that ensure fairness and stability properties in ISS. In practice, the contributions presented in this work are proved to aid firms and policy-makers for evaluating, coordinating, and allocating costs in industrial symbiosis. Our formal frameworks lead to practical tools with commercialization potentials and are validated through various sessions with industrial firms. We argue that this work is the first attempt-and can be a motivation for further approaches-on practical application of MAS technologies in the context of industrial symbiosis as it presents MAS-oriented methodological foundations for ISS development and elaborates on various open problems. 


\section{Contents}

I INTRODUCTION

I.I Background: Industrial Symbiosis from a Multiagent Perspective . . . . 2

I.I.I Topic Motivation: Low-Level Characteristic Points . . . . . . . 3

I.I.2 Topic Motivation: High-Level Characteristic Points . . . . . . 5

I.2 Three Decision-Making Problems in Industrial Symbiosis ～. . . . . . 7

I.2.I Evaluating Industrial Symbiosis . . . . . . . . . . . . 8

I.2.2 Coordinating Industrial Symbiosis . . . . . . . . . . . 9

I.2.3 Allocating Costs in Industrial Symbiosis . . . . . . . . . 9

I.3 A General Review of Research Gaps . . . . . . . . . . . . . . . . . . . Io

I.4 Research Objectives . . . . . . . . . . . . . . . . . . . . I4

I.5 On the Main Approach and Applied Methodologies . . . . . . . . . . I8

I.6 Outline of the Dissertation . . . . . . . . . . . . . . . I9

2 Evaluating Industrial Symbiosis Opportunities 22

2.I Introduction . . . . . . . . . . . . . . . . . . . . . 24

2.2 Conceptual Analysis and Literature Review . . . . . . . . . . . . . . 26

2.2.I Operational Dimensions of ISRs . . . . . . . . . . 28

2.2.2 Epistemic Dimensions of ISRs . . . . . . . . . . . . 33

2.2.3 ISR Costs and Cost Allocation Mechanisms . . . . . . . . . . 34

2.3 Preliminaries: Formal Definitions and Semantic Machinery . . . . . . . 38

2.3.I Concurrent Epistemic Game Structures . . . . . . . . . . . . 39 
2.3.2 Industrial Symbiosis Setting . . . . . . . . . . . 4I

2.3.3 Shapley-Based Cost Sharing . . . . . . . . . . . 42

2.4 The FlSOF Method . . . . . . . . . . . . . . . . . 43

2.4.I ISR Modeling . . . . . . . . . . . . . . . 44

2.4.2 Promising ISRs and Decision Support Algorithm . . . . . . . 49

2.5 Negotiation Equilibrium and Information Sharing . . . . . . . . . 52

2.5.I ISR Negotiation in Equilibrium . . . . . . . . . . . 53

2.5.2 The Fostering Effect of Information Sharing . . . . . . . . 54

2.6 An ISR Opportunity Filtering Case Study . . . . . . . . . . . . 58

2.6.I Case Description . . . . . . . . . . . . 58

2.6.2 ISR Modeling and Decision Support Algorithms . . . . . . . 59

2.7 Concluding Remarks . . . . . . . . . . . . . . 65

3 Coordinating Industrial Symbiosis Networks 70

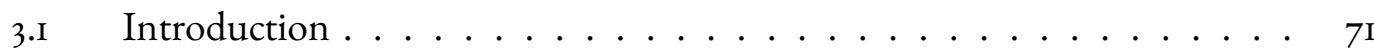

$3.2 \quad$ Conceptual Analysis . . . . . . . . . . . . . . . . . . 74

3.2.I ISN as a Multiagent Practice . . . . . . . . . . . . 74

3.2.2 ISN Regulations as Socioeconomic Norms . . . . . . . . . 75

3.2.3 Evaluation Criteria for ISN Implementation Frameworks . . . . 77

3.2.4 Previous Work at a Glance . . . . . . . . . . . . . 78

3.3 Preliminaries . . . . . . . . . . . . . . . . 79

3.3.I Technical Background . . . . . . . . . . . . 79

3.3.2 Revisiting Previous Work ............. 82

3.4 ISN Games . . . . . . . . . . . . . . . . . . . . . . . . 84

3.4.I ISNs as Cooperative Games . . . . . . . . . . . . . . 84

3.4.2 Benefit Allocation Mechanisms and ISN Games . . . . . . . . 86

3.5 Coordinated ISN . . . . . . . . . . . . . 90

3.5.I Normative Coordination of ISNs ............ 9I

$3.5 .2 \quad$ Coordinated ISNs . . . . . . . . . . . . . . . . . 94

3.5.3 Realized ISNs and Budget-Balancedness . . . . . . . . . . 97

3.6 Concluding Remarks . . . . . . . . . . . . . . . . Ioo 
4 Allocating Transaction Costs in Industrial Symbiosis 103

4.I Introduction and Motivation . . . . . . . . . . . IO4

4.I.I Conceptual Analysis: On the Nature and Dynamics of TC in IS IOS

4.I.2 Institutional Approach: On How to Address the Problem . . . Io8

4.2 Modeling Industrial Symbiosis Institutions . . . . . . . . . . . . . I09

4.2.I Preliminary Notions and Definitions . . . . . . . . . IIO

4.2.2 From Weighted Connectivity Graphs to Cooperative Graph Games III

4.2.3 From Cooperative Games to Industrial Institutions . . . . . . . II5

4.3 A Fair Allocation Mechanism for IS . . . . . . . . . . . . . . . II8

4.3.I On Fairness and Stability . . . . . . . . . . . I20

4.3.2 Reductions and a Tractable Algorithm . . . . . . . . . . I23

4.4 Method Application in the Case Study . . . . . . . . . . . . 2 I26

4.5 Practical Contributions and Open Research Directions . . . . . . . . . I27

5 Review of Contributions, Supplementary Work and Future Research i3I

5.I Synthesis: Revisiting Objectives and a Summary of Contributions . . . . 132

5.I.I Revisiting Objectives .............. I32

5.I.2 Summary of Contributions . . . . . . . . . . 133

5.2 Supplementary Research . . . . . . . . . . . . . . . 134

5.2.I Cooperative and Coordinated Industrial Symbiosis Games . . . 135

$5.2 .2 \quad$ Normative Industrial Symbiosis . . . . . . . . . . . . 136

5.2.3 Emergence and Evolution of Fair Industrial Symbiosis _ . . . . 137

5.2.4 Requirements for Agent-Based Industrial Symbiosis Modeling • 139

5.2.5 Energy-Based Industrial Symbiosis . . . . . . . . . . I40

$5.3 \quad$ Application and Validation Process . . . . . . . . . . . I4I

5.3.I Application: From Decision Support Algorithms to Implemented Tools ....................... I4I

5.3.2 Validation: From Naive Tools to Usable Services . . . . . . . I42

5.4 Future Research Directions . . . . . . . . . . . . . . 143

Appendix A Shapley-Based Cost Sharing for ISRs $\quad$ I48 
Appendix B Balancedness of ISN $_{\Lambda}$

REFERENCES

LIST OF SIKS DISSERTATIONS PUBLISHED SINCE 2OII 


\section{Listing of figures}

2.I ISR's Operational Dimensions . . . . . . . . . . . . . . . . . . 29

2.2 ISR's Epistemic Dimensions . . . . . . . . . . . . . . . . . . . . . . . . . . . . . . . . . . . . . .

$2.3 \quad$ ISR's States and Possible Transitions . . . . . . . . . . . . . . . 47

$2.4 v$ Value for POM's Potential ISR Implementations. . . . . . . . . . . . 64

2.5 $v$ Value for PBB's Potential ISR Implementations. . . . . . . . . . . . 64

$2.6 v$ Value for BTS's Potential ISR Implementations. . . . . . . . . . . 65

3.I ISN and Coordinated ISN . . . . . . . . . . 76

4.I Connectivity graph of the involved firms in the IS cluster . . . . . . . II3 
Albert Einstein says: "the measure of intelligence is the ability to change". And it was my mother, Sara, who showed me that adaptability is the key to success.

In his last lecture, Randy Pausch said: "a lot of people want a shortcut. I find the best shortcut is the long way, which is basically two words: work hard." And it was my father, Kazem, who taught me to work hard for what I want to achieve.

To Sara and Kazem, my role models in life. 



\section{Preface}

At THE TIME OF THIS WRITING, human kind is facing difficult decisions on: how to deal with environmental challenges, how to ensure that technological advancements are in-line with the social good, and how to foster global development in a way that technological advancements can be of use for achieving global growth in a sustainable manner. Such questions are not simple questions for which a fast-thinker or a fast-thinking algorithm (in the sense of [Kahneman, 20II]) would be sufficient. They call for decision support tools tailored to address the subtleties of the questions. In specific, the third question-on whether technology can provide solutions for environmental challenges-is of my interest. Although most of our current wealth is due to technological advancement, we cannot deny that the way we used technology also created new problems.

In my view, the way forward would be to use technology itself as a means for solving the problem. Following this view and the enlightening perspective of [Pinker, 2018], I argue that unused capacities of formal frameworks and technological advancements in (distributed) artificial intelligence and multiagent systems can be of use as reasoning tools to evaluate the viability of innovative business models for industrial collaborations and to foster the implementation of environmentally-friendly practices.

In 20I5, I applied for a $\mathrm{PhD}$ position at the University of Twente, supported by the European Union's Horizon 2020 funding, to investigate the use of agent-based computing and game-theoretic methods to foster circular economy practices. Through the last four years, I studied dynamics of Industrial Symbiosis as a collaborative concept that aims to reduce the material and energy footprint of industrial firms by circulating reusable resources. Implementing such practices is distant from the traditional ways that firms (in particular in the process industry) conduct their business. Thus we need transitional business models and tailored decision support tools for evaluation, coordination, and allocation of costs/benefits in industrial symbiosis systems. In this book, I will present the research that has been conducted in the context of the SHAREBOX Project [SHAREBOX, 2019]. But before presenting the body of the work, I like to thank institutes and individuals who helped me through the process. 
SHAREBOX [SHAREBOX, 2019], the project leading to this work, has received funding from the European Union's Horizon 2020 research and innovation programme under grant agreement No. 680843. I thank all the partners involved in the SHAREBOX project for their constructive feedback through the course of the project.

My PhD supervisors, Prof. Dr. Henk Zijm, Prof. Dr. Jos van Hillegersberg, and Dr. Devrim Murat Yazan are those who gave me the chance to start this line of research and supported me through the ups and downs of the journey. Dear Henk, I learned a lot from you and am grateful for being a student of you. Our discussions on mathematical proofs are of a kind that I will miss. And for sure, this book would be much less readable without your comments. Dear Jos, your support through my $\mathrm{PhD}$ years was strong and encouraging. We started teaching together much before beginning to conduct research together. You gave me the confidence to teach my research to the students and showed me how one can learn to teach but also teach to learn. Dear Devrim, your efforts and enthusiasm were crucial in bringing the SHAREBOX Project to the University of Twente and in making it a successful project. Together, we experienced the challenges of interdisciplinary research and learned that highimpact research needs hard working. I learned a lot by having application of my research in mind and by being sensitive to important subtleties that raise if we really want to apply science in real-world. For four years, almost every Friday at $3 \mathrm{pm}$, we had a meeting to chat, learn, and improve. I think it will take me some time to get used to having no Friday meetings. Thanks for your kind and encouraging support.

IN MY GRADUATION COMMITTEE, I like to acknowledge the constructive feedback received from: Prof. Dr. Vito Albino, Prof. Dr. Mehdi Dastani, Prof. Dr. Tatiana Filatova, Prof. Dr. Karina Gibert, and Dr. Martijn Mes. I am grateful for having you as committee members and thank you for your time and encouraging comments.

In summer 20i9, Prof. Nick Jennings accepted me as a guest researcher to visit him and conduct research at Imperial College London. Dear Nick, my stay at Imperial extended the way I look at multiagent systems research. I learned a lot from you and am grateful for your support.

My accompanying paranymphs during the PhD defense, Dr. Elahe Hadavi and Dr. Nils Knofius, helped me a lot with defense arrangements and also provided valuable hints for a strong defense. Thanks to both of you for your friendly support.

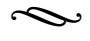


Department of Industrial Engineering and Business Information Systems (IEBIS) is a nice, encouraging, multidisciplinary, and friendly place for working, teaching, and conducting research. I acknowledge the support of all my IEBIS colleagues. In specific, I like to thank Elke and Hilde (for their administrative support and patience); Guido and Luca (for their fruitful collaboration in the context of the SHAREBOX project); Ahmad and Chinatan (for their support at early stages of my PhD); Abhishta, Atil, Iqbal, and Miha (for bringing joy to our common office); Berend R. and Reinoud (for their insightful comments and critical thinking); Derya, Eduardo, Engin, and Wouter (for being a good example on how to conduct good research and proceed in academia); Asad, Berend A., Berry, Martijn K., Rogier, and Sebastian (for our nice and warm conversations, scientific discussions, and coffee-time jokes); and Andrej, Arturo, Sajjad, and Sjoerd (for sharing their experience on how to go through the $\mathrm{PhD}$ journey).

Vahid Yazdanpanah, November 2019, Enschede, The Netherlands. 

In general, I feel if you can't say it clearly you don't un-

derstand it yourself.

$$
\text { John R. Searle }
$$

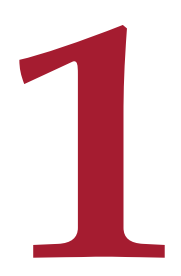

\section{Introduction}

WE BEGIN WITH presenting the background and a multiagent systems perspective on industrial symbiosis, elaborating on three aspects and decision making problems in this topic, introducing research objectives, and explaining the set of applied methodologies as well as the outline of the dissertation. 


\section{i.I Background: Industrial Symbiosis from a Multiagent Perspective}

As a response to recent environmental concerns on reducing the exploitation and discharge of (pure) resources, the idea to circulate reusable resources (also known as the practice of circular economy [Kirchherr et al., 2017]) gained attention in both academia as well as industry ${ }^{\mathrm{I}}$ Industrial Symbiosis Systems (ISS) are green/waste-free implementations of the concept of the circular economy in the context of industrial relations [Chertow, 2007; Yazan et al., 2016]. As an example, consider three firms $A, B$, and $C$ that may have the chance to reuse each other's wastes. For instance, $A$ 's excess steam can be used in $B$ 's turbines, $B$ 's waste water can be used for cooling in $C$, and $C$ 's sludge can substitute a primary input of $A$. Exploiting such an opportunity may lead to financial as well as socio-ecological gains. As ISSs are merely focused on circulating non-commoditized resources, e.g., waste material and energy, waiting for traditional market structures to materialize and successfully implement an ISS would be a guaranteed failure ${ }^{2}$. Thus, ISSs have to be well-designed to be sustainable and in some cases its stability requires the introduction of external monetary incentives [Yazdanpanah et al., 2018]. As Eric Maskin says: "The market is no god-it cannot solve every problem".

To substantiate the novelty of the topic, we present a set of low-level characteristic points of ISS and then elaborate on two high-level aspects—that build upon the first category. In each part, we show how this topic is related to-but distinguished from-adjoining topics.

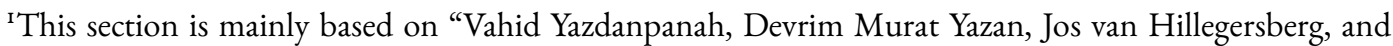
Mehdi Dastani. An introduction to engineering multiagent industrial symbiosis systems: Potentials and challenges. Presented at 7 th International Workshop on Engineering Multi-Agent Systems (EMAS 2019), Montreal, Canada, I3th-I4th May, 2019a" [Yazdanpanah et al., 2019a] and "Vahid Yazdanpanah, Devrim Murat Yazan, and W. Henk M. Zijm. Self-governing industrial symbiosis. (Working Paper), 2orgb” [Yazdanpanah et al., 2019b].

${ }^{2}$ In a more concrete sense, given an environmentally desirable collaboration of $A, B$, and $C$, the allocation of collectively obtained benefits could be problematic in case any firm (group) can gain more by defecting the ISS. 


\section{i.i.I Topic Motivation: Low-Level Characteristic Points}

Non-Commodity Resources: In an ISS, the resources based on which the firms are aiming to establish relations are mostly categorized as "resources with no market". In a sense, the whole idea behind implementing the concept of circular economy is to circulate-instead of to discharge- what can be used but has no (traditional) market. In this vein, having no market categorizes such resources as non-commodity (in the terminology of [Roth, 2015]). Building a business relation based on a non-commodity makes a great distinction between ISS and traditional forms of commodity-based relations. Basically, it avoids using price and price-based techniques for decision-making problems and directs us towards other operational parameters (e.g., the quantity of resources or the set of established regulations that may foster/bind the implementation of the relation).

Loosely-Connected Value Chains: Industrial symbiosis is different from value chain merging as firms remain independent but loosely connected entities. While in the full merging of value chains, the aim is to reach a well-optimized newly-merged entity, the focus of industrial symbiosis is on well-optimized techniques for identification, evaluation, implementation, and governance of the relation(s). In the language of the multiagent systems community, such systems of collaboration can be seen as virtual multiagent organizations/institutions [Criado et al., 2009; Rodriguez et al., 20II].

Normativity of Outcomes: Normativity of ISS refers to the argument that: "not all forms and potential implementations of industrial symbiosis are environmentally/socio-economically desirable”. And there might be some desirable implementations that are not economically 
feasible. This on the one hand highlights the need for norm-aware methods to recognize such implementations and on the other hand, asks for norm-aware coordination techniques to enforce desirable and suppress undesirable collaborations (see [Andrighetto et al., 20I3] for a comprehensive account on norms and normative multiagent technologies).

Potentially Long-Term and Temporally Evolving Relations: As elaborated earlier, industrial symbiosis is not merely a merging process but focuses on establishing a (potentially, long lasting) relation. Note that establishing a new relation requires investments and involves operational costs. So, in most cases, durability of a relation is among firms' concerns. Roughly speaking, firms prefer to implement a relation that lasts-aiming to avoid renegotiating new relations for each and every transfer of reusable resources. Moreover, an ISS operates within the temporally dynamic industrial context (with respect to costs, demands, and supply). Thus, engineering attempts to ensure ISS strength, stability, and fairness have to take into account the dynamics of such systems over time. In brief, building upon potentially long-term industrial synergies, such dynamic relations are distinguishable from single-shot auctioning transactions and have a temporal dimension.

Strategic Aspects and Interdependencies: We explained that-in an ISS-firms remain separate but become interdependent entities. And ideally such relations are dynamic and long-lasting. Thus, the initiation and stability of relations in an ISS critically depends on the dynamics of power and the firms' strategic abilities. In principle, firms act with respect to their preferences and strategic potentials-which in turn defines their potential for success $^{3}$. Dismissing this aspect leads to (agent-based) models that represent all firms at the

\footnotetext{
${ }^{3}$ See $[$ Miller, $198 \mathrm{I}]$ for the interrelations between preferences, power, and success.
} 
same (power) level and abstract from the reality of industrial organizations. Such aspects can be explicitly represented using game forms and semantic structures (e.g., see [Alur et al., 2002; Van der Hoek and Wooldridge, 2003]) able to capture the dynamics of power, executable plans/strategies, and firms' potential collaborative outcomes, i.e., to specify and analyze "what strategy ensures what situation for what group of firms".

In general, the literature on industrial symbiosis is rich in addressing case-specific issues. However, we observe that focusing on implementing specific cases hardly results in generalizable solution concepts. E.g., focusing on single-shot I-I relations dismisses the temporal as well as strategic (coalition-formation) dynamics in ISS. Moreover, we observe a lack of generic methods-able to capture high-level organizational and behavioral aspects-for designing and coordinating ISS.

\section{i.i.2 Topic Motivation: High-Level Characteristic Points}

The above-mentioned characteristic points correspond to a perspective in multiagent systems research that: (I) is aware of behavioral semantics of the system (in contrast to syntactic process optimization approaches) and (2) sees organizational concepts as its first-class system modeling objects. Below, we elaborate on this perspective and discuss its relevance for industrial symbiosis modeling.

On Behavior-Aware Organizational Economics: In a purely utilitarian perspective in the economic theory, the assumption is that an agent's choice of action is formed independent of those of others, dismissing the potential interactions among the agents [Dignum, 20I8; Johnson et al., 2017]. While the evolutionary perspective [Hamilton, 20I7] addresses temporality of choices and captures the ability of agents to observe the earlier choices of oth- 
ers, modeling well-structured organizational settings and the formation of collective decisions in such virtual entities necessitates an explicit representation of the behavior of the organization and its structural constraints. This resulted in the introduction of behavior-aware models - inspired by behavioral economic theory [Thaler, 2016] —into the line of research on organizational economics [Barney and Hesterly, 2006]. In such an integration, models are both behavior-aware (i.e., able to capture the evolution of the systems) and organizational (i.e., interaction protocols, collective preferences, and authority structures are well-defined).

Behavior-Awareness in Industrial Symbiosis: In the context of industrial symbiosis, the focus is on design and operation of collaborative relations among traditionally disjoint industrial enterprises with the aim of keeping reusable resources in their value chains [Chertow, 2007; Yazdanpanah et al., 2019e]. To that end, collaborative clusters form an industrial organization where each firm on the one hand has the autonomy to operate with respect to its individual preferences and on the other hand has to comply with organizational rules. Such a dualism corresponds to the principle of "separation of concerns" and generalizability of the organizational framework by separating the qualities of agents ${ }^{4}$ from those of the organization [Boissier and Van Riemsdijk, 2013]. This allows dealing with the preferences of agents as temporal variables - in contrast to developing the organization with respect to a fixed set of specific firms with stable preferences.

Then a question — that links this high-level point to a previously-discussed aspect-is: “why do we need behavior-aware industrial symbiosis models?". The short answer is, behavior-

${ }^{4}$ Through the course of this work, we may refer to firms as agents-following the convention in computational economics. Hence, we see any industrial symbiosis organization as an environment that supports the collaborative interaction of a set of autonomous decision-makers in charge of the involved firms. This way, we dismiss the decision-making processes within each firm and focus on the inter-firm decision processes. 
awareness is required because IS organizations are temporally evolving entities. If IS was a single-shot transaction, one could simply aim for identifying optimal matches and make the transaction with no need for behavior-aware technologies. Temporality of the IS organization also makes it distinguishable from waste-management frameworks as in the latter, wastes will be seen—and dealt with—disconnected from the firms while in IS, they will be considered as reusable resources based on which firms are establishing new forms of long-term collaborative relations.

Organizational Aspects of Industrial Symbiosis: Behavior-aware models are required but not sufficient for IS if they dismiss the so-called "sociality" concerns [Dignum et al., 20I4]. Basically, social agents make decisions not only based on their individual preferences but also considering what is expected from them on the collective level. In the context of industrial symbiosis organizations, sociality can be manifested in terms of protocols for sharing operational costs, firms' expectations from a relation, or conditions that limit the performance of the IS organization as a whole (e.g., in-place monetary instruments such as taxes or subsidies).

Following the presented topic motivation points, we elaborate on three decision-making processes in industrial symbiosis—as focal points of this dissertation - and present decision problems for which we have developed multiagent decision support tools.

\section{i.2 Three Decision-Making Problems in Industrial Symbiosis}

Moving from general aspects that characterize multiagent industrial symbiosis systems as a field of study with various open problems, challenges, and potentials, here we focus on spe- 
cific operational phases of industrial symbiosis and elaborate on three decision problems in industrial symbiosis.

\section{I.2.I Evaluating Industrial Symbiosis}

The first phase in the operationalization of industrial symbiosis (IS) is to identify "who can work with whom"-also known as the industrial symbiosis identification problem. To address this, there exist various information systems (e.g., [Gatzioura et al., 2018, 2019]) to recommend to firms that provide/require a resource, a list of opportunities to negotiate IS relations with firms that require/provide the resource in question [van Capelleveen et al., 20I8]. Following this phase, industrial firms have to decide whether — and based on what priorityto negotiate a particular opportunity. This is a complex decision process known as the industrial symbiosis evaluation problem. In brief, the question is: among the set of recommended opportunities, which are promising for a firm to pursue to the negotiation phase? Given the multidimensional nature of IS relations and having no traditional market price (for resources that are mainly a form of non-commodity), decision-makers have to base their decisions on operational aspects, e.g., on the business model of industries, physical quantity matching, and possible presence of competitors/regulations. This results in a complex decision problem, in need of sound and context-aware decision support tools 5 .

\footnotetext{
${ }^{5}$ Reducing the material and energy footprint of firms is directly linked to sustainability gains and fits their business model with respect to Corporate Social Responsibility (CSR) goals [Jones, 1980]. But the question is: will it result in a sufficient cost reduction to compensate the opportunity costs that firms may face to focus on industrial symbiosis as a novel business model? We observe, in real-life practices, that if firms apply systematically verified decision support tools for making decisions in various phases of industrial symbiosis, the practice is not only a sustainable choice but also a profitable one. In other words, they can evaluate such propositions in a systematic manner. This way, we aim to support the decision processes to motivate firms to spend less time and resources for implementing the transition of their core business towards a circular economy (see [Burke and Logsdon, 1996] for a similar approach towards linking CSR and financial viability).
} 


\section{I.2.2 Coordinating Industrial Symbiosis}

While the IS evaluation problem is concerned with how firms can distinguish bad from acceptable and good from better, in coordinating IS we are looking at a network of industries as a whole. Such a view would be appropriate if one is either in the position of an industrial cluster manager or a regulatory agent. In both cases, the decision-maker may apply various policies and is interested to see which policies satisfy her goals (i.e., lead to the implementation of the set of collaborations that she deems desirable). This situation leads to questions on how, and by what mechanisms, the decision-maker can enforce a given set of desirabilities. Boundaries, properties, and requirements of such mechanisms characterize the so-called industrial symbiosis coordination problem. Note that the coordination problem in this sense is closely related to implementability, i.e., to see coordination mechanisms as a means for making some class of IS relations implementable. For instance, a relation that is desirable from the policy-maker's point of view might be economically unfeasible from a firm's perspective. In such a case, allocating monetary incentives in a wise manner can change the dynamics. In a general view, who gets what and how ${ }^{6}$ defines the structure of a potential solution for the IS coordination problem.

\section{i.2.3 Allocating Costs in Industrial Symbiosis}

Finally, we move to the phase that an industrial symbiosis relation or a set of interconnected relations are already implemented and functional. Then one of the questions, concerned with keeping the relation(s) stable, is about the allocation of costs such that each firms con-

\footnotetext{
${ }^{6}$ This is to acknowledge that the phrase we used here is based on the title of a book by Alvin Roth [Roth, $2015]$.
} 
tribution is taken into account ${ }^{7}$. For allocating costs that are directly linked to physical interactions, e.g., transportation and treatment/recycling costs, we can rely of physical properties such as the travelled distance or consumed energy. However, one class of transaction costs has an institutional nature and is not merely a result of physical activities. So, although cost allocation mechanisms are well-studied in computational economics, their tailoring for the context of industrial symbiosis calls for methods to capture the physical as well as institutional contributions of involved firms in IS networks. That is the cost allocation problem in industrial symbiosis.

\section{I.3 A General Review of Research Gaps}

Although a detailed motivation and review of the literature-specific to each of the abovementioned decision problems-is presented in the upcoming chapters, a generic discussion on the current state of the art, and on knowledge and research gaps, is presented here as a motivational step. In the following, we briefly discuss research gaps in relation to the abovementioned decision problems (see the upcoming chapters for a more detailed review of the literature and topic motivation). This way, we review the general challenges that are observable in the IS literature and elaborate on the scientific stance upon which our objectives as well as methodological approach are grounded.

\footnotetext{
${ }^{7}$ Note that we are not claiming that cost allocation in industrial symbiosis is fully independent of the abovementioned problems in IS. Basically, all the three decision problems for which we develop decision support tools are related but distinguishable. For instance, at the evaluation level there is an outlook towards a cost allocation scheme that is satisfactory for both the involved firms (without knowing the precise cost allocation). This is necessary for evaluating whether a potential form of IS implementation is mutually promising. In the following chapters, such links and relations will be discussed in details.
} 
Lack of Generic Operations-Oriented IS Evaluation Methods. Having a set of identified IS opportunities—normally as tuples of potential firm matches-we lack analytical methods for evaluating them. Reviewing the IS literature, we see contributions that focus on evaluating the efficiency of an already established IS network, e.g., based on energy consumption or the use of raw material [Berkel et al., 2009; Geng et al., 2012; Sun et al., 2017]. Such approaches are generally based on empirical analysis of a data set, on the flow of energy or physical material in a given case. While their results may significantly improve the state of the studied cases [Geng et al., 20I4; Karlsson and Wolf, 2008; Soratana and Landis, 20II], generalizing them for platforms in which various industrial sectors are involved-as a focal point of the SHAREBOX project [SHAREBOX, 2019] -is not recommended. Basically, to evaluate potential IS matches and analyze various prospects, such retrospective analysis methods (in which a data set of network behavior is given) are not applicable in principle. In brief, we lack prospective IS evaluation techniques that are generally applicable for ranking collaboration opportunities. We later specify how such an objective can be approached using an operations oriented perspective and by applying decision analysis techniques (and not data-driven methods).

Lack of Precise and Verifiable IS Coordination and Policy Support Tools. The need for coordination of a collaborative system implies the aim to suppress some undesirable or enforce some desirable properties-the latter is also referred to as collective goals [Kling, 1991; Malone, 1988]. This is known in the common language as "enabling to work together properly" [Merriam-Webster Online, 2019]. It is also the case for IS coordination that a set of desiderata is expected to be ensured through the coordination mechanisms such that it can operate properly. The challenging part is the way one specifies what "proper" 
means. Descriptions of desiderata are well-studied in the IS literature and are even linked to the coordination problem and to the need for "appropriate" coordination techniques (see e.g., [Boons and Baas, 1997; Desrochers, 2004; Mirata, 2004]). However, we observe inherited vagueness when one applies natural language for desiderata specification. We argue that using the terms "fair", "resilient", or "efficient" [Chopra and Khanna, 20I4; Weidema, 20oo; Wolf et al., 2007] do not convey a precise and verifiable property unless one provides a precise definition in an expressive language. If a coordination method is going to be employed by policy-makers, they should be able to verify whether the desired properties hold in their context of application-and necessarily as a result of applying the coordination methods. We later build on this idea and show how the integration of formal specification, normative techniques, and verification methods improves the state of art in the IS literature.

Lack of Allocation Methods for Collective Transaction Costs in IS. In general, the allocation of collective transaction costs, among the involved firms in an IS network, is a dismissed topic in the IS literature. Although the reduction of transaction costs is identified as one of the drivers behind the implementation of IS practices, they are generally seen as individual costs for which allocation is irrelevant [Chertow and Ehrenfeld, 20I2]. However, thanks to technological innovations and the ubiquitous use of web-based e-commerce systems for IS [Grant et al., 20I0; van Capelleveen et al., 20I8], transaction costs for IS market search, negotiation, and monitoring contracts (known as transactional friction [Williamson, 1985]) are aggregated in the costs for establishing and maintaining IS information systems. Albeit a fraction of transaction costs for IS is still inherently individual, the collective part is in need of allocation methods. The other aspect that characterizes transaction costs as a special category, different from other forms of IS operational costs (such as the IS transporta- 
tion or treatment costs) is the institutional dimension of transaction costs [Silverman, 1999; Williamson, 1979]. In principle, transaction costs are intangible costs related to building and maintaining a relation and not directly linked to physical aspects (like the flow of material or energy in the case of IS transportation, or the physical process of recycling or filtering a resource as a form of IS treatment). This gives IS transaction cost a special dual nature-of being crucial for the flow of the resources but not directly derivable based on the physical properties of the resource. We later provide more elaboration on our understanding of the nature of collective IS transaction cost and motivate the applied methodologies for developing mechanisms for its allocation.

In principle, we argue for the use of formal languages to abstract from specific cases, to enable generalizability and to avoid intrinsic vagueness of descriptive specifications. Moreover, by adopting an agent-oriented viewpoint and employing modeling frameworks as well as solution concepts from multiagent systems research, agency and autonomy of decisionmakers are captured by design. Industrial symbiosis systems are multiagent systems in nature. Hence, we consider it a natural choice to apply methods from multiagent research to study or engineer such systems. In specific, if one is focused on the interaction of micro-level decisions from decision-makers with a degree of autonomy, ${ }^{8}$ agent-based computing methods for outcome evaluation, for behavior coordination, and for cost allocation, are advantageous to other system analysis methods such as factor-based methods from system dynamics modeling [Forrester, 1958] or event-based methods from discrete event systems [Gordon, 1961]. This perspective-on appropriateness of formal methods and agent-oriented methods-is

\footnotetext{
${ }^{8}$ We follow the standard view in agent-based research and see agents as autonomous decision-making entities with the capacity to observe and react to the environment as well as the capability to communicate with other entities in order to pursue their goals proactively [Wooldridge and Jennings, 1995].
} 
a basis for this dissertation, embedded in its research objectives and reflected in the applied methodologies.

In the following section, we present the focal point of this dissertation as developing multiagent models, methods, and ultimately usable decision support services to guide firm managers and policy-makers through the above-mentioned phases of industrial symbiosis realization.

\section{I.4 Research Objectives}

As discussed in previous sections, there are different decision-making procedures involved in industrial symbiosis realization. In this dissertation, we mainly focus on three of those: on evaluating industrial symbiosis opportunities, on coordinating industrial symbiosis networks, and on allocating transaction costs in industrial symbiosis. In each of the three problems, decision-making is not straight forward—as it involves various agents and may result in a spectrum of outcomes - thus requires systematically verifiable support tools to aid the decision-makers.

In a practical sense, the aim of this work is to provide generic decision support tools that are not limited to a specific case while at the same time expressive enough to enable a decision analyst - in an industrial firm or a policy-making unit, say to fill in the required (case-specific) information and receive support on a given case.

We earlier elaborated on how the three problems (on evaluation, coordination, and cost allocation) relate to each other and discussed their chronological order. Below, we present more detailed research objectives that we aim to achieve in each chapter of the dissertation. 
Evaluating Industrial Symbiosis Opportunities (Chapter 2): As sketched earlier, when dealing with non-commodity resources for which price is not a valid determinator, the evaluation of industrial symbiosis opportunities calls for methods that are able to take into account multiple operational aspects. Moreover, it is crucial to capture the dynamics of knowledge and epistemic uncertainties inherited in such decisions. Then, our first step towards supporting IS evaluation decisions is to capture the operational as well as epistemic dimensions of the problem.

Research Objective I. Capture the operational as well as epistemic aspects of the IS opportunity evaluation problem.

Based on the operational and epistemic dimensions, a formal framework to represent and reason about the potential outcome of various decisions is required.

Research Objective 2. Tailor a multiagent formal framework for representing and reasoning about the behavior of industrial symbiosis relations.

Given such a framework, potential outcomes of different (collective) decisions can be represented (we call each outcome a potential implementation of the IS opportunity). Based on such a formal structure, we can develop decision support methods that guarantee firms to reach outcomes that are promising for involved parties.

Research Objective 3. Develop a formal decision support tool for evaluating different implementations of an IS relation. 
Coordinating Industrial Symbiosis Networks (Chapter 3): The focus of the IS coordination problem is on IS enabling techniques for cases that are collectively desirable but are not necessarily feasible for all the involved parties, e.g., cases where-to realize the relation-one or more firms should suffer economically. In such cases, even if collective economic benefits are foreseeable, lack of stability and/or fairness may lead to non-cooperative decisions. Thus, the first step towards addressing the coordination problem is to model IS networks in a formalism that enables reasoning about fairness and stability properties.

Research Objective 4. Model industrial symbiosis networks as multiagent cooperative games (which enables reasoning about fairness and stability properties).

Then, to enable coordination of IS networks, such a multiagent representation should be integrated with regulations and policies, as normative instruments that correspond with the real-life practice of IS.

Research Objective 5. Integrate regulative rules and normative socioeconomic policies with rulebased representation of industrial symbiosis games.

Finally, we investigate the properties of applicable regulatory rules that enable policymakers to ensure the implementability of desired IS collaborations in a fair and stable manner.

Research Objective 6. Develop policy-support tools for generating coordinative regulations that ensure the implementability of desirable industrial symbiosis networks (in a fair and stable manner). 
Allocating Transaction Costs in Industrial Symbiosis (met in Chapter 4): Here, we focus on the nature of collective transaction costs in industrial symbiosis and develop a contextualized allocation mechanism that guarantees game-theoretical fairness and stability properties. We see collective transaction cost in industrial symbiosis as a dual-nature concept with physical as well as institutional dimensions. Relying on John Searle's account of institutional economics [Searle, 2005] and the literature on graphical cooperative games (see e.g., [Driessen, 2013; Kearns, 2008]), we state the following research objective.

Research Objective 7. Formulate a realistic game-theoretical model that incorporates the physical as well as institutional dimensions of collective transaction costs in industrial symbiosis.

While the idea to take into account each agent's contribution provides a basis for allocating the costs, we lack methods for defining the value of each and every coalition of firms. Such an input is crucial for applying standard fair allocation mechanisms.

Research Objective 8. Introduce a quantitative measure that captures the physical and institutional contribution of firms in industrial symbiosis networks.

Finally, using the introduced measure and building on a standard cost allocation technique, we present an allocation method that ensures fairness and stability properties, and that is rooted in the literature on fair division mechanisms.

Research Objective 9. Develop a fair and stable transaction cost allocation mechanism for industrial symbiosis. 


\section{I.5 On the Main Approach and Applied Methodologies}

Due to the interdisciplinary nature of the topic and in order to address various dimensions of the research objectives, we apply a variety of methodologies-mainly from multiagent systems research, computational economics, industrial organization, institutional theory, and temporal epistemic logics.

As a basis of our modeling perspective, we follow the idea that the formal representation of multiagent systems [Wooldridge, 2009] enables systematic reasoning about their evolution and fosters coordinating their complex behavior ${ }^{9}$. Applying this perspective in the context of industrial symbiosis results in multiagent formal models, coordination methods, and decision support tools for industrial symbiosis systems. We integrate some (not yet integrated) multiagent methods, showed a new application domain for multiagent techniques, and contextualize them for evaluating, coordinating, and allocating costs, in industrial symbiosis.

Using a Temporal Logic for IS Evaluation: To address research objectives I-3, we use (a tailored form of) Concurrent Epistemic Game Structures (CEGS) as a representation and reasoning framework, rooted in temporal strategic multiagent logics. Using CEGS, we model the behavior of industrial symbiosis relations and integrate quantitative values-that represent potential implementations of a relation-into CEGS. This results in a decision support algorithm that we argue to be one of the first cases of real-life application of logic-based

\footnotetext{
${ }^{9} \mathrm{It}$ is noteworthy that - as a common practice in most forms of model-based research - in our approach to address these problems, we assume a level of knowledge about the set of involved agents or their potential acts. To capture such knowledge, we see that data-driven techniques can be combined with our line of research in order to come up with an estimation, e.g., of probable actions for agents. We follow Pearl [Pearl, 20I8; Pearl and Mackenzie, 20I8] and argue that such a combination is advantageous compared to purely model-free approaches; specifically in the context of decision support tool development.
} 
techniques in the context of industrial symbiosis.

Cooperative Game Theory and Normative Methods for IS Coordination: To address research objectives 4-6, we mainly use a rule-based form of cooperative gamesnamely Marginal Contribution Nets (MC-Nets) — to enable the integration of industrial symbiosis games with regulative rules and normative policies. So, building on a game-theoretic foundation, the combination of rule-based normative multiagent techniques [Andrighetto et al., 2013] leads to a policy-support tool for ensuring the implementability of desirable industrial symbiosis collaborations.

Computational Economics and Institutional Theory for IS Transaction Cost Allocation: Finally, to address research objectives 7-9, we apply a perspective from institutional economics [Searle, 2005] to reason about the nature of transaction costs and to motivate the introduction of a quantitative index for integrating its institutional and physical aspects. Building on this foundation, we use a graph-theoretical notion and a gametheoretical concept to capture the institutional and physical aspects, respectively. Building on this, we apply the Shapley value [Shapley, 1953] as a standard value allocation method in computational economics to formulate our transaction cost allocation mechanism for industrial symbiosis.

\section{i.6 Outline of the Dissertation}

The remainder of this dissertation is structured as follows. In Chapter 2, we approach the IS evaluation problem and meet research objectives $\mathrm{I}-3$. This results in FISOF and $\mathrm{FISOF}^{+}$, two decision support methods for evaluating industrial symbiosis opportunities. In Chapter 3 , 
we shift our attention towards the IS coordination problem and meet research objectives 46. In practice, the presented method in this chapter is a policy-support tool which ensures the implementability of a set of desirable IS collaborations for a regulatory agent (or an industrial cluster manager). In Chapter 4 , we focus on the cost allocation problem in IS and specifically address the allocation of transaction costs. This results in meeting research objectives 7-9 by introducing a fair and stable allocation method that captures the institutional as well as physical aspects of firms' contributions in industrial symbiosis networks. We conclude in Chapter $\varsigma$ by: revisiting research objectives and summarizing the contributions, presenting supplementary research outcomes, elaborating on the application of our methods and the validation process, and finally discussing open problems and future research directions. 

Intuitive diagnosis is reliable when people have a lot of relevant feedback. But people are very often willing to make intuitive diagnoses even when they're very likely to be wrong.

Daniel Kahneman

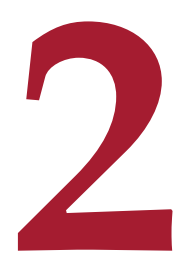

\section{Evaluating Industrial Symbiosis}

\section{Opportunities}

Industrial Symbiotic Relations (ISRs), as bilaterally cooperative industrial practices, are emerging relations for exchanging reusable resources among production processes of in- 
dependent firms. In ISRs, firms can enjoy mutual environmental, social, and economic benefits. Due to similarities in aim and functionality of ISRs and the concept of Circular Economy (CE), it is expected that ISRs play a major role in implementing the CE philosophy in the context of industrial production. However, industrial firms generally lack analytical tools tailored to support their decisions whether-and based on what priority-to negotiate a particular ISR opportunity, selected from a set of potential alternatives. This question is the main focus of the decision support method developed in this chapter, that we call the "industrial symbiosis opportunity filtering" problem. The key economic factor that influences the decision of firms to reject or negotiate an ISR in real-life scenarios, is the total cost-reduction/benefit that they may enjoy in case the ISR would be implemented. In case they evaluate that a sufficient benefit is obtainable, they see the opportunity as a promising one and pursue to contract negotiations. Following this observation, we take an operationsoriented stance and provide a Formal Industrial Symbiosis Opportunity Filtering method (FISOF in short) that: (I) takes into account the key operational aspects of ISRs, (2) formalizes ISRs as industrial institutions using semantic structures adopted from the multi-agent systems literature, and (3) enables the evaluation of ISR opportunities using implementable decision support algorithms $s^{\mathrm{I}}$. In practice, the FISOF method and its algorithms can be integrated into industrial symbiosis frameworks to support firms in the process of ISR evaluation. We also illustrate how information sharing enables the use of collective strategies to overcome epistemic limitations and provide a decision support algorithm that is able to capture all mutually promising ISR implementations.

\footnotetext{
IThe content of this chapter is mainly based on "Vahid Yazdanpanah, Devrim Murat Yazan, and W. Henk M. Zijm. FISOF: A formal industrial symbiosis opportunity filtering method. Engineering Applications of Artificial Intelligence, 81:247-259, 2019e” [Yazdanpanah et al., 2019e].
} 


\section{I INTRODUCTION}

The concept of Circular Economy (CE) and its application in the industrial context opposes the traditional linear production approaches that mainly take primary inputs, produce outputs, and dispose wastes. The circular economy is characterized by circulating reusable resources (e.g., waste material and energy) among production processes and maintaining them in the value chains [Pearce and Turner, 1990; Yuan et al., 2006]. One step further, Industrial Symbiotic Relations (ISRs), as bilaterally cooperative industrial practices, are emerging relations for exchanging reusable resources among production processes of independent industrial firms [Chertow, 2007; Yazan et al., 2016]. So, due to similarities in aim and functionality of CE and ISR, it is reasonable to expect that ISRs play a major role in implementing the CE philosophy in the context of industrial production (see [Andersen, 2007; Sertyesilisik and Sertyesilisik, 2016]). In ISRs, involved firms can enjoy mutual environmental, social, and economic benefits. Moreover, ISRs have a positive influence on both the resilience of firms (as they seek alternative resource suppliers) and the efficiency in exploitation of available resources (as they substitute traditional primary inputs with wastes) [Fraccascia et al., 2017b].

As reviewed in [Mannina et al., 2019; van Capelleveen et al., 20I8], there exist various information systems for identifying ISR opportunities. These platforms are mainly platforms that recommend to a firm that provides/requires a resource, the opportunity to negotiate ISRs with firms that require/provide the resource in question. However, industrial firms generally lack analytical tools tailored to support their decisions whether-and based on what priority - to negotiate a particular ISR opportunity. Roughly speaking, the question is which ISR opportunities, identified by a recommender system, are sufficiently promising 
for a firm to pursue to the negotiation phase? This question is the main focus of the decision support method that we developed to address the "industrial symbiosis opportunity filtering” problem. Due to the multidimensional nature of ISRs, such a decision support method has to regard multiple operational aspects, e.g., the business model of industries, physical quantity matching, and possible presence of competitors/regulations. Although there exist methods for analyzing each of these dimensions in ISRs², filtering ISR opportunities calls for methods that are able to take into account multiple operational aspects and can also deal with epistemic uncertainties inherited in such decisions. Then, the first step to support such decisions is to provide formal methods able to capture both the behavior of such relations and their potential economic outcomes.

In real ISR scenarios, the key economic factor that influences the decision of firms to reject or negotiate an ISR is the obtainable cost reduction (or benefit) that they may enjoy in case the ISR would be implemented [Albino et al., 2016]. Accordingly, in case they evaluate that a sufficient cost reduction is obtainable, they see the opportunity as a promising one and pursue to contract negotiations ${ }^{3}$. Following this observation, we take an operations-oriented stance and develop a Formal Industrial Symbiosis Opportunity Filtering method (FISOF in short) that: (I) takes into account key operational aspects of ISRs, (2) formalizes ISRs as industrial institutions using semantic structures adopted from the multi-agent systems literature, and (3) enables evaluating ISR opportunities using implementable decision support algorithms. In practice, the FISOF method and its algorithms can be integrated into industrial symbiosis

\footnotetext{
${ }^{2}$ For instance, input-output analysis is a standard method for investigating interrelations between firms with respect to the flow and quantity of resources (cf. [Sendra et al., 2007]).

${ }^{3}$ We later discuss that such an operational perspective on ISRs is in-line with Alvin Roth's view on matching markets and the procedure of evaluating the quality of matches in such markets [Roth, 20I5; Roth and Sotomayor, 1992]
} 
decision-modeling frameworks to support firms in the process of ISR evaluation. We also illustrate how information sharing enables the use of collective strategies to overcome epistemic limitations (that each firm may suffer from) and provide a decision support algorithm that is able to capture all the mutually promising ISR implementations (as a basis for ISR negotiations).

The structure of the chapter is as follows. First we introduce an operational perspective on ISRs in Section 2.2. It includes the analysis of operational dimensions of ISRs, the role of epistemic aspects, and main ISR-related costs. The formal preliminaries required for modeling ISRs will be provided in Section 2.3. In Section 2.4, we sketch the FISOF method that includes a model of the behavior of ISRs as an industrial institution and the decision support algorithm. Section 2.5 presents an analysis on the occurrence of ISR negotiations in equilibrium, illustrates the fostering role of information sharing, and ends with an algorithmic method that enables ISR opportunity filtering under distributed knowledge. In Section 2.6, the applicability and performance of our methods are illustrated using a case study. Finally, concluding remarks are presented in Section 2.7.

\subsection{Conceptual Analysis and Literature Review}

In this section, we present an operational perspective on Industrial Symbiosis Relations (ISRs from now on) and analyze various concepts that play a key role in the evaluation, establishment, and operation of such relations. Accordingly, we introduce operational dimensions of ISRs that in later sections frame a formal operations-oriented decision support method for ISRs. 
We describe ISRs as two-member industrial institutions ${ }^{4}$ that correspond to two-sided matching markets [Roth and Sotomayor, 1992]. In matching markets, the procedure of allocating resources involves a "match evaluation" stage. The class of matching markets and their associated economics opposes the traditional category of (merely) price-oriented markets. In the former the focus is on evaluation of potential matches while in the latter, price forms the market and explains its dynamics. Following Alvin Roth [Roth, 2015], we distinguish the situation of standard commodities of which the price can be seen as the main parameter for decision-making from situations in which the transaction is based on non-standard commodities for which the price negotiation is not the first practical stage to operationalize the economic practice. In such cases, prior to the negotiation procedure, involved actors consider whether a given deal, relation, or in general a setting that describes the opportunity for implementing the economic practice, is a reasonable one. This approach, i.e. to model and evaluate specific classes of economic transitions as matching markets, resulted in successful scenarios in various contexts such as bilateral kidney exchange and educational student-institute matching [Abdulkadiroğlu et al., 2005; Roth et al., 2005]. As discussed earlier, ISRs are transactions mainly based on reusable resources, e.g. waste energy and material, which typically do not operate in a commoditized price-driven market. For instance, when a firm manager learns about an ISR opportunity on a specific reusable waste, in most cases there is no standard market for that waste; hence no standard market price to rely on during the evaluation phase. In such a situation, managers seek decision-support tools able to take into account various operational aspects of ISRs for evaluating and narrowing down the set of available ISR opportunities to

\footnotetext{
${ }^{4}$ We use the term "institution" in a broad sense as a multiagent system that operates under a form of agreement between the involved agents. See [Ossowski, 20I2, Part IV] for a comprehensive account on multiagent organizations and institutions.
} 
a set of promising ones. Afterwards, firms may pursue negotiations with the most promising ISR opportunities and (potentially) implement some.

In order to establish a basis for evaluating ISR opportunities ${ }^{5}$, in this section we present an operations-oriented analysis of parameters based on which firms can evaluate an ISR opportunity (Section 2.2.I). Moreover, we address epistemic aspects that influence a firm's evaluation (Section 2.2.2). Such a classification facilitates the process of formalizing ISRs and developing the Formal Industrial Symbiosis Opportunity Filtering (FISOF) method. In brief, FISOF supports a firm's decision on whether a particular ISR opportunity is a promising one (to pursue to the negotiation phase) by taking into account the operational as well as epistemic aspects of the relation.

\subsection{Operational Dimensions of ISRs}

In the following, we discuss operational dimensions of ISRs (illustrated in Figure 2.I) and the structural subtleties that each brings into consideration.

\section{Business-Making Perspective}

First, we discuss ISRs from a business point of view. In particular, we distinguish whether the receiver side of an ISR is going to use the waste as a substitution for one of its traditional primary inputs or if it is going to build its business (e.g., establishing a new production line) directly based on the received resource. We call the latter cases Direct ISRs and the former cases Substitution-based ISRs.

\footnotetext{
${ }^{s}$ As this work is merely focused on the evaluation of ISR opportunities (and not on already implemented ISRs), we may simply say "ISRs" whenever it is clear from the context that we mean "ISR Opportunities".
} 
Industrial Symbiotic Relations

(Operational Dimensions)

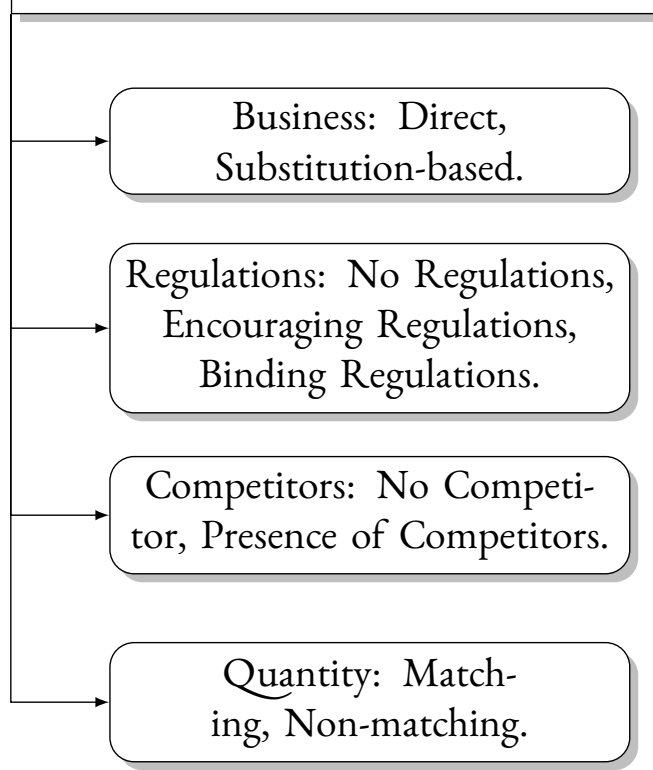

Figure 2.1: ISR's Operational Dimensions

Realizing whether an ISR is direct or based on substitution has both operational and technical consequences for the process of ISR evaluation and decision support. Firstly, concerning operational aspects, in substitution-based ISRs, the receiver firm will decide about the profitability of a potential ISR by considering the trade-off between implementing the ISR and rejecting it. This is basically because the firm is traditionally receiving a primary input from another source and should analyze whether substituting it with the reusable resource (from the ISR in question) is profitable. Secondly, with respect to technical aspects of substitution-based ISRs, the benefits of substituting a traditional primary input with a reusable resource depends on the so-called substitution rate. Intuitively, the substitution rate is the ratio that determines how much of one resource is needed to replace one unit of the other one. For instance, in the cement production industry, one unit of an alternative fuel, 
e.g., Municipal Solid Waste (MSW), may substitute one or more units of coal-as the traditional energy source used in cement industries. We refer the reader to [Albino et al., 20II] for details about the substitution rate and extensive investigations about the use of alternative resources for energy purposes in the cement industry. In further sections, we point out how distinguishing direct and substitution-based ISRs influences the procedure of ISR evaluation.

Presence of Regulations

While we are dealing with reusable resources such as waste material/energy, various binding or encouraging regulations may be in place. Such regulations may exist for the provider/supplier in a potential ISR, the receiver, or both. Moreover, they can be either in the form of binding regulations, e.g., prohibition of discharge/transportation of a particular type of waste, or in the form of encouraging regulations, e.g. awarding tax-reductions or subsidies to the firms that use wastes of other firms as their input.

Some governmental regulations may consider prohibitions for specific resources and bind up discharge for a resource-provider or compel the use of alternative inputs for a resourcereceiver. There might be cases in which receivers are obliged to realize a certain amount of substitution which is driven by environmental regulations. For example, as discussed in [Albino et al., 2OII], a cement company might be obliged to reduce the $\mathrm{CO}_{2}$ emissions caused by coal use, which may serve as a motivation to use alternative energy sources-causing less $\mathrm{CO}_{2}$ emissions. Therefore, we need to consider the extra taxes paid for $\mathrm{CO}_{2}$ emissions or any other sanctions introduced by the government. On the other hand, incentives may be present for waste reduction on the provider side or on the receiver side for reduced primary resource de- 
pletion. Additionally, incentives may exist to encourage circular economic business models [Murray et al., 2015; Pearce and Turner, 1990] as the umbrella concept for the practice of industrial symbiosis. For example, bioenergy producers may accept paying a high price for low energy-density biomass as they receive incentives from governments for producing renewable energy. Similarly, providers can be encouraged for supplying their reusable resources to a certain sector which is being promoted by governments for sustainability reasons.

As discussed in [Desrochers, 2004; Zhu et al., 2007], encouraging incentives can foster the emergence of spontaneous industrial symbiotic relations as they compensate the involved costs (see further sections for a characterization of the main costs in ISRs). Hence, encouraging incentives can improve the profitability level so that involved firms are convinced that the ISR is a promising one, thus start the negotiations and potentially implement the ISR. Regulations in favor of ISRs will generally lead to cost reductions whereas regulations against ISRs may induce additional costs.

\section{Presence of COMpetitors}

The presence of competitors on either or both sides of an ISR affects the ISR evaluation and choice of involved firms [Johanson and Mattsson, 2015; Kochan et al., 1984]. In basic cases, an ISR may be established with respect to a resource for which there exists only one provider and one receiver (in the region, country, or any geographical scope of analysis). On the other hand, for some resources there may exist more than one provider or receiver. Hence, in the ISR evaluation phase, firms mostly face a set of ISR opportunities-and not a single opportunity - to be evaluated. As presented in [Yazan et al., 2012], the dynamics of bargaining power in industrial symbiotic relations is highly dependent on the number of potential 
relations. In general, the higher the number of potential relations, the higher a firm's bargaining power-hence its risk tolerance. One step further, in established relations, ensuring the resilience and stability of the relation against the entrance of a competitor may even require external monetary incentives [Yazdanpanah et al., 20I8].

Moreover, in some cases a potential ISR opportunity might be evaluated as "promising” as long as competitors are not considered but as "non-promising" once these other competitors are taken into account. For instance, when the quantity of a resource, provided by a firm $A$, does not match the amount that firm $B$ requires, $B$ may reject to negotiate the ISR with $A$ (only) if it observes the possibility to establish another relation with a competitor resourceprovider firm $C$-that is able to provide the quantity that $B$ requires.

In principle, the presence of competitors leads to more ISR opportunities for firms to evaluate, potentially negotiate, and implement. Formal representations of these concepts and methods to rank such a set will be presented in further sections.

\section{QuAntity Matching}

The other operational dimension that characterizes an ISR opportunity is the relation between the physical quantity of the reusable resources: produced by the provider and required by the receiver ${ }^{6}$. If the quantity of a produced resource matches the need of another firm, supply meets demand and the ISR (in case of operationalization) experiences a higher level of stability in comparison to non-matching quantities. Several ISR research contributions highlight the importance of quantity matching and aim for reducing the resource disposal by means of finding perfect symbiotic relations in which the produced amount matches the

\footnotetext{
${ }^{6}$ Note that wastes are a form of secondary product—not produced upon demand.
} 
required amount (e.g., [Standing et al., 2008; Yazan et al., 2016]). In [Yazan et al., 2016], matching physical quantities in an Industrial symbiotic Network (ISN) is one of the main conditions for realizing a so called perfect ISN and in [Standing et al., 2008], the authors argue that the unavailability of reliable and consistent quantity data is one of the barriers against the establishment of sustainable production chains as a step towards the circular economy [Pearce and Turner, 1990].

In general, when two quantities match, the resource provider firm can enjoy paying no discharge cost while the receiver firm has no purchase cost for obtaining its traditional input. On the other hand-in non-matching quantities-even after implementing the ISR, provider/receiver firms have to deal with the remaining discharge/purchase costs to compensate the mismatching quantities.

\subsubsection{Epistemic Dimensions of ISRs}

In this section, we focus on the availability of information for decision makers faced with an ISR opportunity ${ }^{7}$. Information is crucial in the process of decision-making to get engaged in an industrial relation. In principle, the more information available for the decision-maker, the more accurate the decision is. [Chertow, 2000] indicates that in successful industrial symbiosis cases in Denmark [Grann, 1997] information availability played a key role. However, in industrial practices, the availability of perfect information is not a reasonable assumption. Considering possibly distant firms and also taking into account the diversity of suppliers/receivers may result in cases where firms are not perfectly informed about the presence of

${ }^{7}$ In this work, we see each firm as a single industrial agent, autonomous in its decision-making. Moreover, we abstract from intra-organizational decision processes and also from cognitive/mental aspects of decisionmaking. 
competitors. With respect to the availability of information about the business-making models and the quantities, assuming perfect information is not reasonable as the firms involved in a potential ISR are independent and autonomous companies that may opt not to fully share information. Accordingly, in our modeling we consider ISR opportunity evaluation under imperfect information with respect to (I) business observability, (2) market observability, and (3) production observability (Figure 2.2). Under imperfect information some potential implementations of an ISR opportunity may be indistinguishable for a firm. For instance, when a resource-provider firm $A$ is not informed about the business model of a resource-receiver $B$, firm $A$ cannot distinguish between a direct implementation of its ISR opportunity with $B$ and one based on substitution. Similar indistinguishable situations occur when firms lack information about other epistemic dimensions of an ISR opportunity.

Regarding regulations, we assume that all industrial firms are perfectly informed about the presence of regulations. This is reasonable since regulations are publicly available and are introduced by governments ${ }^{8}$. Such regulations involve encouraging incentives or binding rules in favor of, or against a particular ISR opportunity. We later show how firms can reason under imperfect information and also illustrate the advantages of information sharing.

\subsubsection{ISR Costs and Cost Allocation Mechanisms}

Implementing ISRs have economic, environmental, and social benefits. Following [Albino et al., 2016], we believe that economic benefits are the main parameter that affects the decision process of industries to get involved in a potential ISR (see also contributions that aim for minimizing ISR operational costs, e.g., [Montastruc et al., 2013; Rubio-Castro et al.,

\footnotetext{
${ }^{8}$ Such an assumption can be relaxed in future work by considering multiple epistemic levels for industrial agents.
} 


\section{Industrial Symbiotic Relations}

(Epistemic Dimensions)

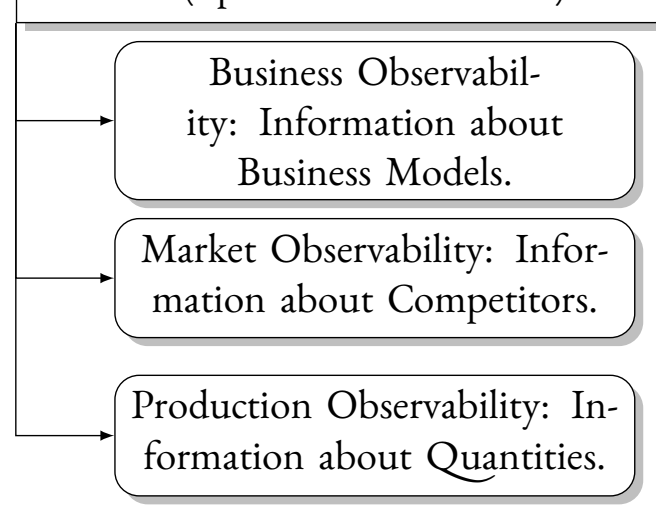

Figure 2.2: ISR's Epistemic Dimensions

20II]). In other words, when a firm evaluates whether an ISR opportunity is sufficiently promising to start the negotiation procedure, it mainly compares the potential case with its current situation. In such an evaluation, firms compute the amount of cost reduction (or benefits) they can enjoy thanks to the implementation of the relation. Roughly speaking, the total cost to operationalize an ISR should be compared with the total cost reductions (or potential benefits) that it brings about-due to its potential to reduce waste discharge cost and traditional-input purchase cost. In the following, we first present the two classes of ISR operational costs: $3 T$ operational costs and profile-specific costs. Secondly, a Shapley-based [Shapley, 1953] method for sharing operational costs among the involved firms will be presented.

\section{T Operational Costs}

According to [Esty and Porter, 1998; Sinding, 2000], the three main operational costs that are involved in an ISR are transportation, treatment, and transaction costs ( $3 T$ costs in short). 
Transportation Cost: The role of transportation costs in the establishment of ISRs and potential cost reductions thanks to implementing one is well-studied in the literature (see [Carpenter and Gardner, 2008; Chen et al., 20I2; Zhang et al., 2015]). For instance, in a case study in [Carpenter and Gardner, 20o8], the transportation costs reduced with $25 \%$ due to closer proximity of the substituting resource. In general, transporting reusable resources can be done via land vehicles, ships, trains, or even combined transportation modes [Zijm et al., 2015], depending on the resource type, geographical boundaries, and whether the resource is categorized as a hazardous one. Moreover, potential partners might decide to invest in implementing new infrastructures, e.g., a pipeline system, and paying the investment cost together. In this work, we abstract from subtleties in the mode of transportation (as discussed by [Guenther and Farkavcová, 20Io]) and assume a standard total cost for resource transportation.

Treatment Cost: In principle, most reusable resources (e.g., waste material and energy) as secondary outputs of a production process first need to be treated. Treatment processes might be sorting, drying, dismantling, liquefaction, gasification, etc, depending on the resource type [Costa et al., 20I0; Lovelady and El-Halwagi, 2009; Magram, 20II]. Moreover, the location of a treatment facility may differ due to the dynamics of treatment costs. For instance, as studied in [Yazan, 2016], there are various options to locate the treatment facility: at the provider firm, at the receiver firm, at a third party specialized on recycling, or even at the initial primary resource provider (that stays in the loop and attempts not to get influenced by the resource substitution procedure). Together, these aspects determine the total costs of the treatment process in any given ISR. 
TRANSACTION COST: In general, transaction costs include the costs of: market research, contract negotiations, coordination, and adaptation to the use of the substituted resource [Dahlman, 1979; Williamson, 198I]. According to [Andrews, 2000; Sharfman et al., 1997], industrial symbiotic practices can lead to a reduction in the total transaction cost over time. As in this work we are focusing on industrial symbiotic relations and not networks with (potentially) diverse sets of transaction costs, we assume a single value for the total transaction cost per symbiotic relation.

\section{Profile-Specific Costs}

The above mentioned $3 \mathrm{~T}$ costs are general operational costs that are common for different forms of ISR (e.g., direct or substitution-based ISRs). In the process of ISR evaluation, in addition to the general ${ }_{3} \mathrm{~T}$ operational costs, a profile-specific cost that should be taken into account for direct ISRs is the total production setup cost that includes the set of costs related to the initiation of a new production line. These costs involve the monetary investments, related costs for production licenses, facilitation costs, and all the costs necessary for initiation of a new production line on the receiver side of a direct ISR.

Moreover, one specific design choice is to formulate the effects of regulations and mismatching quantities in terms of costs. In other words, whenever there exists a regulation that binds a particular ISR, we add a positive cost to our ISR evaluation equations. Analogously, we add a negative cost if an ISR takes place in the presence of incentives in its favor. Finally, the costs due to quantity mismatch will be considered as extra costs for firms. This representation enables a utility-based approach that fosters quantitative analysis of dynamic decisions in ISRs using the rich literature on game theory [Kreps, 1990; Osborne, 2004]. 


\section{ISR Cost Allocation Mechanisms}

As discussed earlier, various industrial symbiosis and case-specific studies see economic benefit (or cost reduction) as the main driver behind industrial symbiotic relations [Jacobsen, 2006; Park and Behera, 20I4; Van Berkel et al., 2009]. In an ISR, the provider firm may enjoy cost reductions by shifting from disposing the resource to a novel symbiotic practice while the receiver firm may enjoy cost reduction in its purchasing cost. The main point is that for an ISR to be implementable, the total ISR operational costs (after integrating monetary incentives and other extra costs) must be less than the firms' costs in case they do not implement the ISR. Thus, methods for allocating the operational costs among firms play a key role in feasibility and long-term stability of such relations.

Reviewing the mature literature on game-theoretic cost-allocation solution concepts [Lemaire, 1984; Lindroos, 2004; Littlechild and Owen, 1973; Lozano et al., 2013; Young, 1985b], the efficiency and rationality of such mechanisms result in cost-allocation methods able to guarantee that players have an incentive to collaborate and remain collaborating. In this work, we employ the tailored Shapley-based cost-allocation method in [Yazdanpanah and Yazan, 20I7] which guarantees both fairness and stability of ISRs over time.

\subsection{Preliminaries: Formal Definitions and Semantic Machinery}

In this section, we first present the formal semantic structure based on which we build the FISOF method, then define the set of variables that represent an ISR setting, and finally illustrate the cost-sharing mechanism that will be employed for allocating costs in our decision support algorithm. 


\subsection{Concurrent Epistemic Game Structures}

To model Industrial Symbiotic Relations (ISRs) and enable systematic reasoning about their behavior, we use Concurrent Epistemic Game Structures (CEGS) [Ågotnes et al., 20I5] as an epistemic extension of Concurrent Game Structures (CGS) [Alur et al., 2002]. In general, CEGS allows modeling any system in which multiple actors/agents are involved and act under imperfect information. Formally, CEGS is a tuple $\mathcal{M}=\left\langle N, Q, A c t, \sim_{1}, \ldots, \sim_{n}, d, o\right\rangle$ where:

- $N=\left\{a_{1}, \ldots, a_{n}\right\}$ is a finite, non-empty set of agents;

- $Q$ is a finite, non-empty set of states;

- Act is a finite set of atomic actions;

- $\sim_{a} \subseteq Q \times Q$ is an epistemic indistinguishability relation for each agent $a \in N$ assuming that $\sim_{a}$ is an equivalence relation (i.e., $q \sim_{a} q^{\prime}$ means that states $q$ and $q^{\prime}$ are indistinguishable to $a$ );

- multi-function $d: N \times Q \mapsto \mathcal{P}(A c t)$ defines the set of actions available for each agent in each state (we require that the same actions be available to an agent in indistinguishable states, i.e., $d(a, q)=d\left(a, q^{\prime}\right)$ whenever $\left.q \sim_{a} q^{\prime}\right)$;

- and $o$ is a deterministic transition function that assigns the outcome state $q^{\prime}=o\left(q, \alpha_{1}, \ldots, \alpha_{n}\right)$ to state $q$ and (for $\left.\alpha_{i} \in d\left(a_{i}, q\right)\right)$ to an $n$-tuple of actions $\alpha_{1}, \ldots, \alpha_{n}$ that can be executed by $N$ in $q$.

Having an ISR modeled in a CEGS-based multi-agent system, one can reason about states that involved firms can bring about in case they follow specific forms of decision-making 
strategies ${ }^{9}$. The following notions enable representing and reasoning about such strategies and their outcomes under imperfect information ${ }^{\mathrm{IO}}$.

Group Epistemic Relations: When agents form groups, their epistemic limitations (in the collective level) will be represented as follows. Let $G \subseteq N$ be a group of agents. Following [Fagin et al., 1995], we model the notions of distributed knowledge by means of derived relation $\sim_{G}^{D}=\bigcap_{a \in G} \sim_{a}$. Intuitively, this notion describes the epistemic limitations of a group in terms of states that are indistinguishable for all the group members-represented by the intersection of indistinguishability relations.

Successors And Computations: To represent the relation among possible states, potential chains of states, and their dynamics, we have the following. For two states $q$ and $q^{\prime}$, we say $q^{\prime}$ is a successor of $q$ if there exist actions $\alpha_{i} \in d\left(a_{i}, q\right)$ for $i \in\{1, \ldots, n\}$ such that $q^{\prime}=o\left(q, \alpha_{1}, \ldots, \alpha_{n}\right)$, i.e., in $q$, agents in $N$ can collectively guarantee that $q^{\prime}$ will be the next system state. A computation of a given CEGS $\mathcal{M}$ is an infinite sequence of states $\lambda=q_{0}, q_{1}, \ldots$ such that for all $\ell>0$ we have that $q_{\ell}$ is a successor of $q_{\ell-1}$. We refer to a computation that starts in $q$ by a $q$-computation. Moreover, for $\ell \in\{0,1, \ldots\}$, we denote the $\ell^{\prime}$ th state in $\lambda$ by $\lambda[\ell]$. Finally, $\lambda[0, \ell]$ and $\lambda[\ell, \infty]$ respectively denote the finite prefix $q_{0}, \ldots, q_{\ell}$ and infinite suffix $q_{\ell}, q_{\ell+1}, \ldots$ of $\lambda$.

\footnotetext{
${ }^{9}$ In upcoming sections, we present a formal account of such a model. Intuitively, potential implementations of a given ISR opportunity will be represented by states in $Q$ and firms' available actions (e.g., to opt for a direct or substitution-based business model) will shape the set of actions Act.

${ }^{\text {Io }}$ References to elements of $\mathcal{M}$ should be seen as elements of a CEGS $\mathcal{M}$ that is modeling a particular multiagent system, e.g., we write $Q$ instead of $Q$ in $\mathcal{M}$.
} 
Strategies and Outcomes: Strategies can be seen as a form of decision-making agenda for agents. Formally, an imperfect information strategy for an agent $a \in N$ is a function $\zeta_{a}: Q \mapsto$ Act such that, for all $q \in Q:(\mathrm{I}) \zeta_{a}(q) \in d(a, q)$ and (2) $q \sim_{a} q^{\prime}$ implies $\zeta_{a}(q)=\zeta_{a}\left(q^{\prime}\right)$. For a group of agents $G \subseteq N$, a collective strategy $Z_{G}=\left\{\zeta_{a} \mid a \in G\right\}$ is an indexed set of strategies, one for every $a \in G$. Then, out $\left(q, Z_{G}\right)$ is defined as the set of potential $q$-computations that agents in $G$ can enforce by following their corresponding strategies in $Z_{G}$.

\subsubsection{Industrial Symbiosis Setting}

We discussed in Section $\mathbf{2 . 2}$ that firms face costs either in case they opt to implement an ISR (including $3 \mathrm{~T}$ operational costs) or if they continue their traditional practice (i.e., discharge/purchase costs for the resource-provider/-receiver firms). Moreover, they may enjoy monetary incentives (in the form of subsidies or taxes) in either cases. This results in a trade-off for each firm when they are reasoning about an ISR opportunity. Accordingly, a firm considers an ISR promising if it has the potential to bring about a sufficient benefit (or cost reduction). That means, if an ISR can lead to cost reductions more than a specific (subjective) value, then the firm opts to pursue to the negotiation phase. To represent the set of above mentioned cost parameters that reflect the so called industrial symbiosis setting, we employ a value profile structure. Formally, we model an industrial symbiosis setting between resource provider firm $A$ and resource receiver firm $B$ as a tuple $\mathcal{S}=$ $\left\langle O, T_{A}, T_{B}, R_{A}, R_{B}, \epsilon_{A}, \epsilon_{B}, E_{A}, E_{B}\right\rangle$ where:

- $O$ is the total $3 \mathrm{~T}$ operational cost for implementing the ISR;

- $T_{A}$ is the traditional resource discharge cost for firm $A$; 
- $T_{B}$ is the traditional input purchase cost for firm $B$;

- for $i \in\{A, B\}, R_{i}$ is the amount of monetary incentive that $i$ receives for implementing the ISR;

- for $i \in\{A, B\}, \epsilon_{i}$ is the minimum amount of obtainable cost reduction that $i$ considers sufficient to pursue to ISR negotiations;

- for $i \in\{A, B\}, E_{i}$ is the summation of $i$ 's extra costs due to mismatching resource quantities and individual investments.

We highlight that in case an ISR is considered as an "undesired" relation from the legislative point of view (e.g., when an ISR is against environmental standards), the applicable amount of tax/penalty can be represented as a negative value for $R_{i}$.

\subsubsection{Shapley-Based Cost Sharing}

As discussed in Section 2.2.3, the implementation of ISRs includes various costs that ought to be shared among the involved firms. Then, one main factor to ensure the long term stability of the relation is the fairness of the employed cost sharing method ${ }^{\mathrm{II}}$. In the following, we recall a cost sharing mechanism, developed in [Yazdanpanah and Yazan, 2017], that guarantees the Shapley-based notion of fairness and preserves its desirable properties, i.e., efficiency, symmetry, dummy player, and additivity [Shapley, 1953].

According to [Yazdanpanah and Yazan, 2017], the allocation of the total 3 T operational cost for implementing an ISR between firms $A$ and $B$ is fair and stable only if it takes into

\footnotetext{
IISee [Yazdanpanah and Yazan, 2017; Yazdanpanah et al., 20I8] for game-theoretic evaluations of this claim and [Yazan et al., 2017b] for agent-based simulation results on this account. Moreover, see Appendix A for a brief mathematical derivation, as well as some intuition behind the notion of Shapley-based fairness in allocating costs in IS relations.
} 
account the dynamics of their traditional costs (i.e., the costs if they opt not to implement the ISR $)^{\mathrm{I2}}$. Then, formally, the fair cost share for firm $i \in N=\{A, B\}$ is equal to $\frac{1}{2}[O+$ $\left.T_{i}-T_{N \backslash\{i\}}\right]$ where:

- $O$ is the total 3 T operational cost for implementing the ISR;

- and $T_{i}$ is the traditional cost for firm $i$.

Note that cost sharing only applies to $3 \mathrm{~T}$ operational costs (and not to firms' extra costs $\left.E_{i}\right)$. This is based on the assumption that firms only share the costs related to resources that are contributing to an ISR and not for the excess resource that should be discharged/purchased due to mismatching quantities or for a firm's individual investment (e.g., to purchase a required facility that will become a firm's property regardless of the ISR).

In the next section, we present the FISOF method and illustrate how the Shapley-based cost sharing mechanism, values that represent firms' costs or preferences, and the epistemic game structure that models the ISR's behavior can be integrated.

\subsection{The FISOF Method}

The Formal Industrial Symbiosis Opportunity Filtering method (FISOF) consists of the following components:

- Institutional Behavior Modeling

- Industrial Symbiosis Settings

- Cost Sharing Mechanism

${ }^{\mathrm{I}}$ In the game-theoretic language, a fair cost sharing considers the marginal contributions of involved agents to the cost game [Shapley, 1953]. 


\section{- Decision Support Algorithm}

While the first three components contribute to modeling the ISR as an industrial institution (in Section 2.4.I), the fourth component focuses on practicality by providing a decision support algorithm (in Section 2.4.2) that generates the ranked list of promising ISR opportunities for a firm.

\subsection{ISR MODELING}

In order to have a realistic representation of a potential ISR, we use (I) Concurrent Epistemic Game Structures (CEGS) to model its institutional behavior, (2) a set of values that represent all the potential industrial symbiosis settings, and (3) a cost sharing mechanism that allocates the operational costs to involved firms. In the following, we first discuss these three elements in detail and then introduce the ISR model as an industrial institution.

Institutional Behavior Modeling: As discussed in [Yazdanpanah and Yazan, 20I7], industrial symbiotic relations can be seen as games in which involved agents (i.e., industrial firms) cooperate to materialize benefits collectively but also compete to obtain a larger share in the total benefit individually. This results in a form of coopetition [Bengtsson and Kock, 2000]. For such a form of industrial institution, we require mechanisms to ensure the fairness of the value-sharing. Otherwise, the stability of the institution will be questionable. While [Yazdanpanah and Yazan, 2017] addresses this problem under the perfect information assumption-using solution concepts from cooperative game theory-we relax this assumption, model ISRs' behavior under imperfect information, and combine solution concepts from cooperative game theory with concurrent game structures. 
Industrial Symbiosis Settings: Dynamics of costs, regulations, quantities, and type of business model play a key role in a firm's decision to consider an ISR as a promising one (to pursue the negotiation). For example, when a resource-receiver firm aims to start a new production line based on a waste material, it may have higher expectations than when it simply aims to substitute a traditional input (of its established production line). Therefore, it is reasonable to allow different ISR settings (as discussed in Section 2.3.2) in different ISR implementations. We further elaborate how such dynamics can be represented in the FISOF method by taking into account ISR settings (instead of a unique ISR setting).

Cost Sharing Mechanism: We discussed above that cost values may change with respect to operational dimensions of an ISR. This directly affects the total operational cost of an ISR and accordingly each firm's share. Thus, we localize the Shapley-based cost sharing mechanism with respect to the outcome of agents' actions and employ our Shapley-based allocation as the principle solution concept to ensure fairness and stability in ISRs.

Accordingly, we define the ISR model as an industrial institution.

Definition I (ISR Model). We say an ISR institution is a tuple $\mathcal{I}=\left\langle\mathcal{M}, q_{0}, \mathcal{S}_{Q}, \Phi\right\rangle$ where:

- $\mathcal{M}=\left\langle N, Q\right.$, Act, $\left.\sim_{1}, \sim_{2}, d, o\right\rangle$ is a two-person concurrent epistemic game structure;

- $q_{0} \in Q$ is a uniquely marked state that represents the initial situation of the institution;

- $\mathcal{S}_{Q}=\left\{\mathcal{S}_{q} \mid q \in Q \backslash\left\{q_{0}\right\}\right\}$ is the indexed set of industrial symbiosis settings (as formulated in Section 2.3.2), one for every $q \in Q \backslash\left\{q_{0}\right\}$;

- and function $\Phi: N \times Q \backslash\left\{q_{0}\right\} \mapsto \mathbb{R}$ is the Shapley-based cost sharing mechanism that ensures the fairness and stability of the institution. For any pair $i \in N$ and 
$q \in Q \backslash\left\{q_{0}\right\}$, we have that $\Phi(i, q)=\frac{1}{2}\left[O+T_{i}-T_{N \backslash\{i\}}\right]$ where $O$ and $T_{i}$ are derived from $\mathcal{S}_{q}$

In an ISR institution $\mathcal{I}$, the behavior of the institution is modeled using a two-person GEGS $\mathcal{M}=\left\langle N, Q, A c t, \sim_{1}, \sim_{2}, d, o\right\rangle$ where: $N$ consists of two agents (representing the two firms involved in $\mathcal{I}$ ); $Q$ is the set of all possible institutional states (representing all the possible implementations of the ISR); Act is the global set of actions that are available to firms (representing all the possible decisions that firms may take); $\sim_{i}$ is the indistinguishability relation for agent $i \in N$ (representing epistemic limitations of firms with respect to possible implementations of the ISR); $d$ is the multi-function that determines the local set of actions that are available to each firm in each state (representing all the possible decisions that each firm may take in each state); and $o$ is the transition function that determines the next state of the institution given the current state and the joint action profile of agents in $N$ (representing the evolution of the ISR institution as the result of agents' joint decisions).

The following example illustrates a scenario to show how an ISR opportunity can be modeled as an industrial institution. In Section 2.6, we analyze a realistic case study to show how a more complex ISR opportunity can be modeled and evaluated using the FISOF method.

Example I (An ISR Scenario). Imagine a case where an industrial symbiosis platform already identified the ISR opportunity between firms $A$ and $B^{\mathrm{I} 3}$. In this scenario, $A$ 's discharge cost is 5 utils $^{\mathrm{I4}}, B$ 's traditional purchase cost is 10 utils, and the total 3 T operational costs for implementing a direct and a substitution-based ISR are 13 and 10 utils, respectively. Moreover, according to regional regulations, $B$ enjoys 3 utils of incentive if it implements the relation

\footnotetext{
${ }^{13}$ In principle, when a firm produces a waste that another firm listed as its required resource, industrial symbiosis platforms consider this as a potential ISR and suggest it to both firms.

${ }^{14} \mathrm{~A}$ util can be any form of transferable utility, e.g., say a util is one thousand Euros.
} 
while $A$ can enjoy no encouraging incentives. With respect to expectations, $A$ prefers to pursue negotiations for implementing either a direct or a substitution-based ISR only if it gains at least 1.5 utils. But for $B, 1.5$ utils is only sufficient for a substitution-based ISR while it expects 2.5 utils for a direct one (as in the latter case $B$ needs to invest in some required facilities which cost 1 extra util). Finally, it is not observable to $A$ whether $B$ uses the resource to substitute an input or to establish a direct ISR business (in case the two firms do not share information on the intentions of $B$ ).

This scenario can be modeled by the ISR institution $\mathcal{I}=\left\langle\mathcal{M}, q_{0}, \mathcal{S}_{Q}, \Phi\right\rangle$ where in $\mathcal{M}$ : $N=\{A, B\}, Q=\left\{q_{0}, q_{\text {dir }}, q_{\text {sub }}\right\}$, Act $=\{\operatorname{dir}$, sub $\}, \sim_{A}=\left\{q_{\text {dir }}, q_{\text {sub }}\right\}, \sim_{B}=\emptyset$, $d(a, q)=$ Act for $a \in N$ and $q \in Q$, and transition function $o$ is as illustrated in Figure 2.3, e.g., the arrow from $q_{0}$ to $q_{d i r}$ with the label $\langle d i r, s u b\rangle$ says that the system goes from $q_{0}$ to $q_{s u b}$ if $A$ and $B$ execute $d i r$ and sub, respectively.

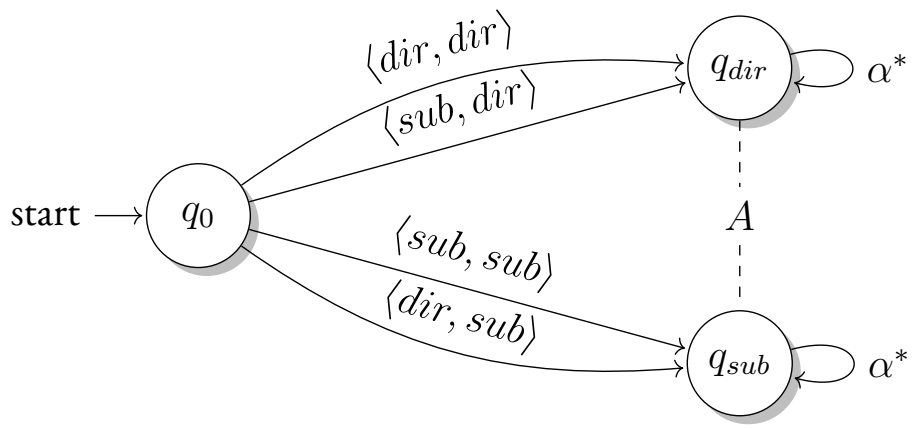

Figure 2.3: ISR's States and Possible Transitions: State $q_{0}$ represents the initial situation in which the ISR is not materialized. In $q_{d i r}$ and $q_{s u b}$ the direct and substitution-based ISRs are implemented, respectively. Moreover, dir and $s u b$ refer to the act of opting to implementing a direct and substitution-based ISR, respectively, while $\alpha^{*}$ refers to any action profile possible. Finally, the indistinguishability of states $q_{d i r}$ and $q_{s u b}$ to $A$ is represented with a labeled dashed line between the two states.

The other elements of this ISR institution, i.e, $\mathcal{S}_{Q}=\left\{\mathcal{S}_{q_{d i r}}, \mathcal{S}_{q_{s u b}}\right\}$ and $\Phi$, are as follows. The industrial symbiosis settings $\mathcal{S}_{q_{d i r}}$ and $\mathcal{S}_{q_{s u b}}$ are equal to $\langle 13,5,10,0,3,1.5,2.5,0,1\rangle$ 
and $\langle 10,5,10,0,3,1.5,1.5,0,0\rangle$, respectively. Finally, with respect to values in these industrial symbiotic settings, we have that $\Phi\left(A, q_{\text {dir }}\right)=4, \Phi\left(A, q_{\text {sub }}\right)=2.5, \Phi\left(B, q_{\text {dir }}\right)=9$, and $\Phi\left(B, q_{s u b}\right)=7.5$.

The main purpose behind modeling ISR opportunities as industrial institutions is to enable reasoning about their behavior and to provide operational semantics to managers of the involved firms ${ }^{\mathrm{I}}$. For instance, in the above ISR scenario, firms are interested to learn about ISR states (i.e., potential implementations of the ISR opportunity) that are in-line with their preferences $^{16}$. This can be realized by answering: "which states in $\mathcal{I}$ satisfy firm $i$ 's minimum expected cost reduction $\epsilon_{i}$ ?".

Definition 2 (Promising States). Let $\mathcal{I}=\left\langle\mathcal{M}, q_{0}, \mathcal{S}_{Q}, \Phi\right\rangle$ be an ISR institution, $i \in N$ be an industrial firm, and $q \in Q \backslash\left\{q_{0}\right\}$ be a state (representing a potential ISR implementation). We say $q$ is a promising state for $i$ iff $T_{i}-\Phi(i, q)-E_{i}+R_{i} \geq \epsilon_{i}$ where $T_{i}, E_{i}, R_{i}$, and $\epsilon_{i}$ are derived from $\mathcal{S}_{q} \in \mathcal{S}_{Q}$. Moreover, $\Pi_{i}$ denotes the set of all promising states for $i$.

Simply stated, an ISR implementation (i.e., a state in $Q \backslash\left\{q_{0}\right\}$ ) is a promising one-for a firm-only if it brings about an amount of cost reduction that the firm considers sufficient ${ }^{17}$.

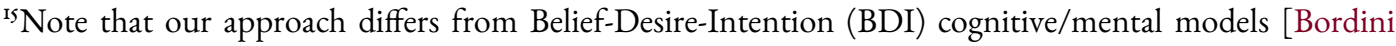
et al., 2006; Rao and Georgeff, 1995]. In principle, BDI-oriented languages focus on modeling and programming the internal reasoning process of agents-i.e., how an agent plans to reach a desirable situation based on its (dynamic) internal beliefs and intentions-while the focus of this contribution is mainly on modeling the evolution of multi-agent system's environment (assuming no access to agent's internal state of mind). As argued in [Yazdanpanah et al., 2019c] — for agent-based industrial symbiosis models -it is not reasonable to assume having access to and control over firms' intra-organizational decision processes (which is a required input for BDI-based models). Therefore, instead of using accessibility (belief, desire, intention) relations to represent the epistemic dynamics of firms, we employ indistinguishability relations and game structures to represent the limited observability of firms on possible implementations of any given ISR opportunity.

${ }^{16}$ Note that a given ISR opportunity may have different potential implementations-represented by CEGS states. For instance, the direct ISR between $A$ and $B$ in state $q_{d i r}$ and the substitution-based ISR in state $q_{s u b}$ are the two possible ISR implementations of the modeled ISR opportunity in this scenario.

${ }^{17}$ We highlight that assigning a negative value to $\epsilon_{i}$ in an ISR setting is valid. Such a value represents a case in which a firm $i$ opts to negotiate an ISR implementation as long as it loses not more than $\epsilon_{i}$. 
For instance, in the ISR scenario (Example I), $q_{s u b}$ is promising for both firms while $q_{d i r}$ is a promising state only for firm $B$. However, due to $A$ 's epistemic limitations, it can not distinguish $q_{d i r}$ from $q_{s u b}$. Moreover, with respect to $A$ 's available actions in $q_{0}$, it has no strategy to avoid $q_{d i r}$. In other words, although a specific implementation of the ISR (in $\left.q_{d i r}\right)$ is a promising one for $B$, the ISR opportunity is not necessarily a promising one for A. This is mainly due to epistemic as well as strategic limitations that firm $A$ is facing-in the process of ISR opportunity evaluation. We later elaborate how information sharing may resolve such situations.

\subsubsection{Promising ISRs and Decision Support Algorithm}

In this section, we build on the notion of promising states and introduce the more general notion of promising ISRs. While the former merely focuses on possible ISR implementations that are desirable for a firm, the latter takes into account firms' epistemic as well as strategic abilities to enforce such implementations. Accordingly, an ISR opportunity would be seen promising by a firm only if it can enforce a promising implementation of the ISR in question.

Definition 3 (Promising ISRs). Let $\mathcal{I}=\left\langle\mathcal{M}, q_{0}, \mathcal{S}_{Q}, \Phi\right\rangle$ be an ISR institution and $i \in N$ be an industrial firm. We say that $\mathcal{I}$ is a promising ISR opportunity for $i$ iff there exits a strategy $\zeta_{i}$ such that for all $\lambda \in \operatorname{out}\left(q_{0}, \zeta_{i}\right)$ and $\ell \geq 1$ we have that $\lambda[\ell] \in \Pi_{i}$. Moreover, the immediate guaranteed value of such $a \zeta_{i}$ in $\mathcal{I}$ is $v\left(\zeta_{i}, \mathcal{I}\right):=\min _{\lambda \in \operatorname{out}\left(q_{0}, \zeta_{i}\right)}\left(\left\{k \mid k=T_{i}-\right.\right.$ $\left.\left.\Phi(i, \lambda[1])-E_{i}+R_{i}-\epsilon_{i}\right\}\right)$ where $T_{i}, E_{i}, R_{i}$, and $\epsilon_{i}$ are derived from $\mathcal{S}_{\lambda[1]} \in \mathcal{S}_{Q}$. Finally, $\Im_{i}$ denotes the set of all promising ISRs for $i$.

Roughly speaking, whether an ISR opportunity (modeled by the ISR institution) $\mathcal{I}$ is 
promising for a firm $i$ depends on all the preconditions that guarantee the existence of a strategy to reach to and stay in an ISR implementation in $\Pi_{i}$.

Example 2 (A Promising ISR?). In the ISR scenario between firms $A$ and $B$, the ISR opportunity is a promising ISR for $B$ because by executing a strategy that starts with either dir or $s u b$, it can enforce a $B$-promising ISR implementation. On the other hand, the ISR is not a promising one for $A$ although there exists an specific ISR implementation that is promising for $A$, i.e., the substitution-based ISR with $B$. In Section 2.5, we show how firms can avoid missing such a mutually beneficial opportunity by sharing information with a secure third-party ISR information system.

The following remark highlights cases where the question whether an ISR opportunity is promising for a firm, can be answered regardless of the firm's abilities but mainly with respect to industrial symbiosis settings.

Remark I (Necessarily Unpromising ISR). Let $\mathcal{I}=\left\langle\mathcal{M}, q_{0}, \mathcal{S}_{Q}, \Phi\right\rangle$ be an ISR institution and $i \in N$ be an industrial firm. If $\Pi_{i}=\emptyset$ then $\mathcal{I}$ is necessarily not a promising ISR for $i$.

Having all the required components for representing an ISR, modeling its institutional behavior, and considering the operational semantics based on which firms can reason about the promisingness of a given ISR, we next formulate the fourth component of the FISOF method. The FISOF method is a practice-oriented model-checking algorithm to supports firms' decisions in the process of filtering ISR opportunities.

Decision Support Algorithm: Using the introduced notion of promising ISR, a particular ISR opportunity can be evaluated. However, this notion is applicable only for cases 
in which no other competing firm exists (i.e., when the evaluation is concerned with a particular ISR opportunity and not a set of opportunities). As we discussed in Section 2.2, in real-life ISR scenarios, a resource-providing/-receiving firm (mostly) has to evaluate multiple ISR opportunities. This is mainly because there exist competitor resource-providing/receiving firms. Then, the ISR evaluation question has two sides: "which ISR opportunities are promising?" and "which are more promising?". To tackle both parts, we use a straightforward transformation of the evaluation problem (i.e., the check whether an ISR is promising) in order to answer the ranking problem (loosely speaking, which ISRs are more promising).

We simply incorporate the possibility of having competitors by enabling the decision support algorithm to receive a list of ISR opportunities for a firm (as the algorithm's input) and to generate a ranked list of promising ISRs for the firm (as the algorithm's output). Such a ranking considers the maximum obtainable cost reduction as the parameter to sort the list of promising ISRs for the firm in question. In other words, the existence of a promising ISR $\mathcal{I} \in \Im_{i}$ for a firm $i$ implies the existence of a nonempty set of strategies that each guarantees a promising ISR implementation for the firm. Then, within this set, an optimal strategy $\zeta_{i}$ would be a strategy that results in the highest value $v\left(\zeta_{i}, \mathcal{I}\right)$ for $i$ (in the promising ISR $\mathcal{I}$ ). We consider this maximum value, denoted by $\vartheta_{i}(\mathcal{I})$, as a property of a promising ISR $\mathcal{I}$ (for firm $i$ ) and employ it as the ranking factor in the model checking Algorithm I.

The FISOF algorithm generates a ranked list of promising ISRs available to a particular firm. Based on such a list, firms can reason about the most-promising ISR opportunities and strategize about the ISR negotiation process. We later go through a run of this algorithm in a case study.

Next, we study the conditions for occurrence of an ISR negotiation and discuss how some 


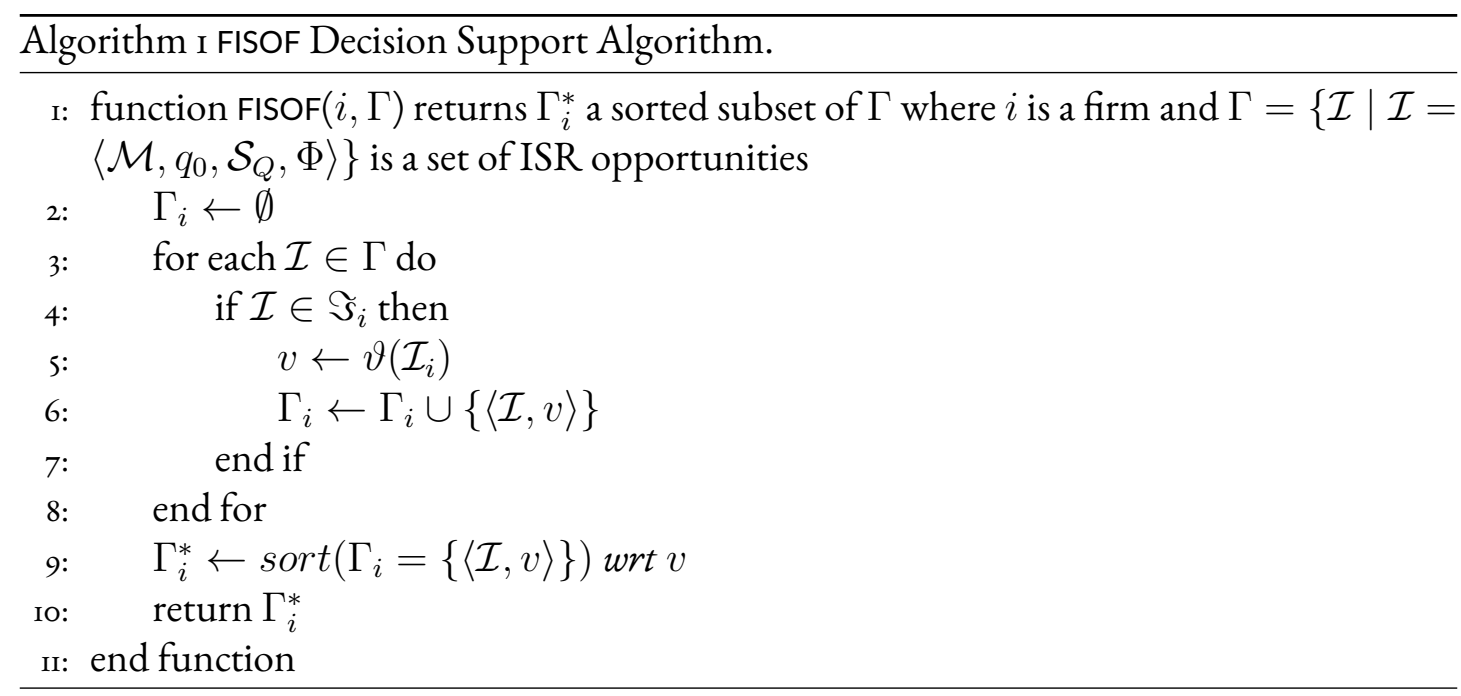

limitations can be resolved using collective strategies that rely on information sharing.

\subsection{Negotiation Equilibrium and Information Sharing}

When firms receive a notice about the potential to establish an ISR, e.g., from an ISR platform that matches firms, the execution of the FISOF algorithm - seeing it integrated into the ISR platform-can show that the ISR is promising: (I) for both, (2) for neither of, or (3) only for one of, the firms involved in the opportunity. Accordingly, firms opt to negotiate the ISR opportunity only if it is a promising one for them. In this section, we first present a game-theoretic analysis on the cases in which the ISR negotiation takes place in a so called Nash equilibrium and then show a resolution for cases where firms can overcome some strategic/epistemic barriers by means of information sharing. 


\subsection{ISR Negotiation IN EQUiLibrium}

Relying on the FISOF method that filters ISR opportunities using their operational properties on the micro-level, we now focus on the macro-level with the aim to analyze the occurrence of the ISR negotiation on a particular ISR opportunity. This is mainly to show when negotiations take place. We assume that, using the FISOF method, firms have sufficient capacities to negotiate with all the promising ISRs and reject any unpromising ISR. In a game-theoretic structure, such meta-level decisions can be presented in a two-person non-cooperative game where firms can either negotiate or reject an ISR opportunity. The following proposition shows that the ISR negotiation on an ISR opportunity occurs in a Nash equilibrium ${ }^{\mathrm{I} 8}$ only if it is a mutually promising ISR.

Proposition I (ISR Negotiation in Equilibrium). Let $\mathcal{I}=\left\langle\mathcal{M}, q_{0}, \mathcal{S}_{Q}, \Phi\right\rangle$ be an ISR institution. With no prior communication, ISR negotiation on $\mathcal{I}$ occurs in a Nash equilibrium iff $\mathcal{I} \in \Im_{i}$ for all $i \in N$.

Proof. “ $\Rightarrow$ ": In this four state game-as the result of negotiate/reject decisions of two playersthe negotiation (i.e., negotiate-negotiate state) takes place only if both parties opt to negotiate. Assume that the ISR opportunity is not among the promising ISRs for both parties, then it is either unpromising for both or only for one. In both cases, one or both parties opt to reject which contradicts with the premise.

“Æ”: Having $I \in \Im_{i}$ for all $i \in N$ implies that for both forms, $\vartheta(\mathcal{I})_{i}$ is larger than zero, i.e., both can obtain sufficient cost reductions in some implementations of the ISR opportu-

\footnotetext{
${ }^{18}$ The materialization of a situation, as the result of a mutual decision, in a Nash equilibrium [Mas-Colell et al., 1995] implies that no party has rational incentives to deviate from the decision that results in the situation.
} 
nity. Accordingly, both have no incentive to deviate and hence the negotiate-negotiate state would be a Nash equilibrium.

While this result shows the cases where the negotiation takes place ${ }^{\mathrm{Ig}}$, it also illustrates that some mutually beneficial ISRs will not qualify to be negotiated-as a result of epistemic or strategic limitations of individual firms. To see this, we recall the ISR scenario in Example 2. In this scenario, $A$ rejects the ISR due to its inability to distinguish the promising state $q_{s u b}$ (which represents a promising ISR implementation for both firms) from $q_{d i r}$ (which represents an unpromising ISR implementation for $A$ but a promising one for $B$ ). This shows that although $A$ and $B$ can mutually benefit from the ISR, $A$ rejects the ISR opportunity, hence an obtainable cost reduction will be dismissed. A natural solution-supported by empirical results in [Fraccascia and Yazan, 2018] - is to provide a secure information sharing platform with which all the involved firms can share information. This is mainly to delegate the ISR evaluation process to automated processes that can enjoy the so called distributed knowledge [Fagin et al., 1995] among the set of involved delegates.

\subsubsection{The Fostering Effect of Information Sharing}

As we have shown earlier, there might be promising ISR implementations that firms dismiss to negotiate merely due to their lack of information. Roughly speaking, firms opt to reject an ISR opportunity if they cannot individually enforce a promising implementation of it. While sharing sensitive information with other firms is not always realistic in the industrial context, sharing information with a secure multi-agent decision-making platform is a feasible

\footnotetext{
${ }^{19}$ Note that this result is about the selection (filtering) of the most promising symbiotic relationships, and not the coordination of the negotiation process as such.
} 
resolution to this issue. Such a framework can directly use and explore the set of ISR implementations (i.e., the set of all possible promising states in $Q$ ) instead of making the decision to negotiate under epistemic limitations that firms may suffer from.

The next proposition shows that due to monotonicity of power [Holler and Napel, 2004], aggregation of firms in the grand coalition empowers them and makes more states (that represent ISR implementations) collectively reachable.

Proposition 2 (More is More). Let $\mathcal{I}=\left\langle\mathcal{M}, q_{0}, \mathcal{S}_{Q}, \Phi\right\rangle$ be an ISR institution and let $\mathcal{S}_{\text {next }} \subseteq$ $\mathcal{S}_{q}$ be a set of successors of $q_{0}$. Then the set of states that any a $\in N$ can guarantee in $\mathcal{S}_{\text {next }}$ (denoted by $\mathcal{S}_{\text {next }}^{a}$ ) is a subset of the set of states that $N$ can guarantee in $\mathcal{S}_{\text {next }}$ (denoted by $\left.\mathcal{S}_{\text {next }}^{N}\right)$; formally, $\mathcal{S}_{\text {next }}^{a} \subseteq \mathcal{S}_{\text {next }}^{N}$.

Proof. To prove $\mathcal{S}_{\text {next }}^{a} \subseteq \mathcal{S}_{\text {next }}^{N}$, we show that for any individual strategy $\zeta_{a}$ that guarantees a state $q \in \mathcal{S}_{\text {next }}$ (i.e., for all $\lambda \in \operatorname{out}\left(q_{0}, \zeta_{a}\right)$ we have that $\lambda[1]=q$ regardless of what other agents in $N$ choose to do) there exist a collective strategy $Z_{N}$ able to guarantee the same $q$. For any arbitrary $\zeta_{a}$ that guarantees a $q$, we can construct a collective strategy $Z_{N}$ in which $a$ follows $\zeta_{a}$ in $q_{0}$ while other agents in $N$ have arbitrary actions. Such a $Z_{N}$ guarantees $q$. Note that the equality, i.e., $\mathcal{S}_{\text {next }}^{a}=\mathcal{S}_{\text {next }}^{N}$, does not hold necessarily due to the fact that $\sim_{N}^{D} \subseteq \sim_{a}$ for all $a \in N$.

Using Proposition 2, the next theorem illustrates that using distributed knowledge and collective strategies, firms will be able to recognize and immediately enforce any ISR implementation that is mutually promising.

Theorem I (Collectively Enforceable Promising States). Let $\mathcal{I}=\left\langle\mathcal{M}, q_{0}, \mathcal{S}_{Q}, \Phi\right\rangle$ be an ISR institution. Moreover, let $q \in \bigcap_{i \in N} \Pi_{i}$ be a successor of $q_{0}$. If $q_{0} \notin \sim_{N}^{D}$ then there exists a 
collective strategy $Z_{N}$ such that for all $\lambda \in \operatorname{out}\left(q_{0}, \zeta_{N}\right)$ we have that $\lambda[1] \in \bigcap_{i \in N} \Pi_{i}$.

Proof. As $q$ is a mutually promising state (i.e., ISR implementation of $\mathcal{I}$ ), we can prove the theorem by showing the ability of firms to reach $q$. As illustrated in Proposition 2, having that the grand coalition's strategic ability to enforce a successor state is only limited to its epistemic limitation and given that $q_{0} \notin \sim_{N}^{D}$, we have that the two firms in $N$ can collectively enforce $q$ as a mutually promising implementation of $\mathcal{I}$.

This result shows that relying on the knowledge that is distributed among firms, they can collectively make sure that no mutually promising state (i.e., ISR implementation) will be dismissed. Accordingly, we present the $\mathrm{FISOF}^{+}$algorithm-a variation of FISOF-that assumes the availability of distributed knowledge and hence applicability of collective strategies for evaluating a set of ISR opportunities (Algorithm 2).

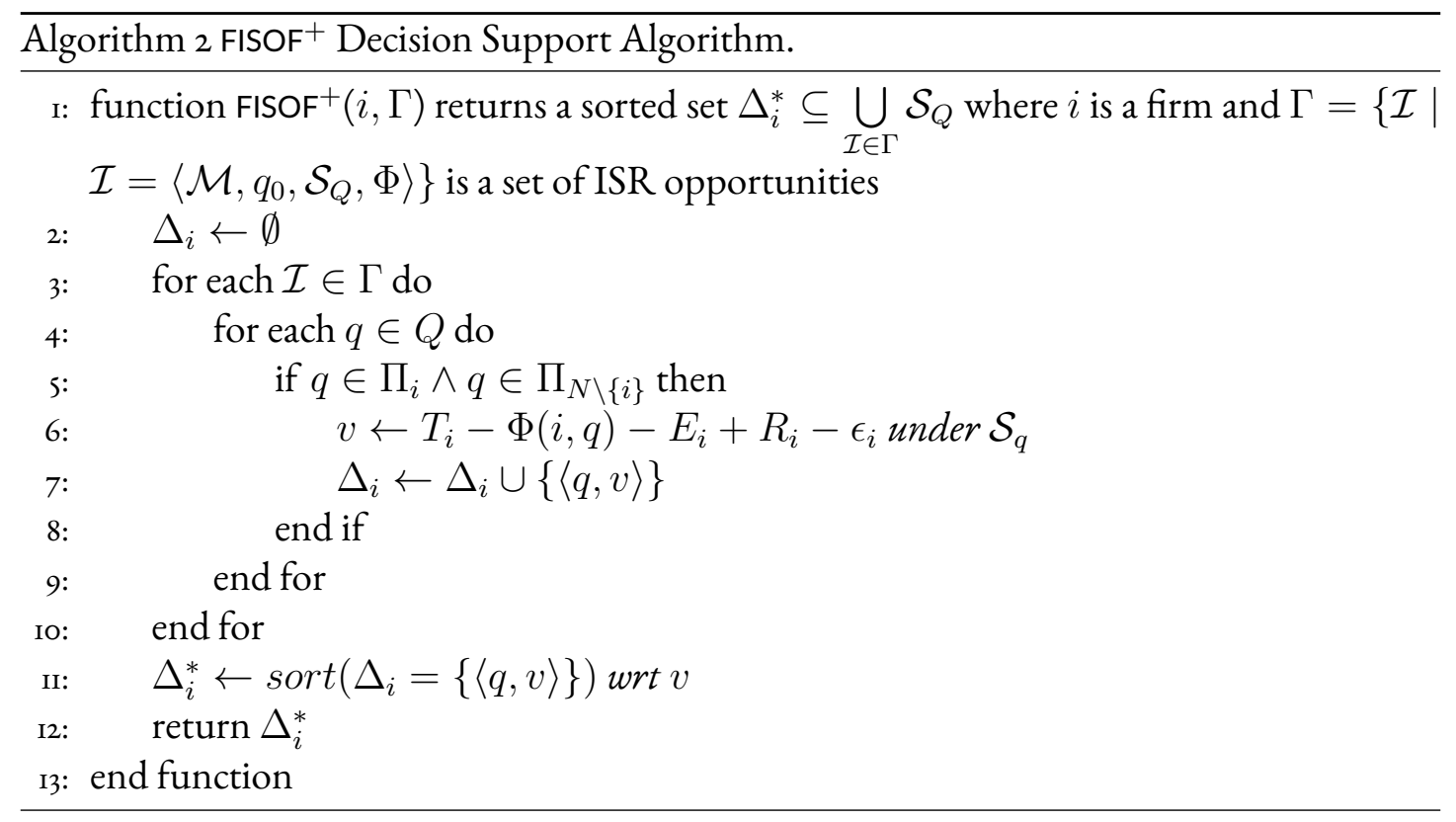

Note that $\mathrm{FISOF}^{+}$takes a set of ISR opportunities as its input and generates a sorted list 
of mutually promising ISR implementations as its output. We discussed earlier that (using FISOF) epistemic limitations of firms result in the occurrence of ISR negotiations only on mutually promising ISRs and illustrated that some mutually promising implementations may be dismissed accordingly. Then, the question is whether using the extended method, i.e., using $\mathrm{FISOF}^{+}$, provides the chance of ISR negotiation on implementations that are dismissed in FISOF. The following theorem shows that using distributed knowledge and collective strategies in $\mathrm{FISOF}^{+}$, we can capture all mutually promising ISR implementations that FISOF covers in addition to those that it may dismiss.

Theorem 2 (FISOF vs. FISOF ${ }^{+}$). Let $\Gamma=\left\{\mathcal{I} \mid \mathcal{I}=\left\langle\mathcal{M}, q_{0}, \mathcal{S}_{Q}, \Phi\right\rangle\right\}$ be a set of ISR opportunities, $i$ be a firm, and $\Lambda_{i}=\bigcup_{\mathcal{I} \in \operatorname{FISOF}(i, \Gamma)}\left\{q \mid q \in \Pi_{i}\right\}$ be the set of promising ISR implementations for $i$ under FISOF. We have that the set of possible ISR negotiations (in equilibrium) under $\mathrm{FISOF}^{+}$includes the set of possible ISR negotiations (in equilibrium) under FISOF, formally that $\bigcap_{i \in N} \Lambda_{i} \subseteq \bigcap_{i \in N} \operatorname{FISOF}^{+}(i, \Gamma)$.

Proof. According to Algorithm 2 (line 5), the results of FISOF ${ }^{+}$includes any mutually promising ISR implementation (possible in $\Gamma$ ). This shows that possible negotiations under FISOF are included in the set of possible negotiations under $\mathrm{FISOF}^{+}$. To prove, we then have to show the inequality of the two sets (i.e., $\bigcap_{i \in N} \Lambda_{i}$ and $\bigcap_{i \in N}$ FISOF $^{+}(i, \Gamma)$ ). Relying on Proposition 2 and Theorem I, we have that the two sets are not equal (in principle) as firms may face epistemic/strategic limitations that avoid them to negotiate on some mutually promising ISR implementations. In particular, they may not start negotiations on an ISR that seems unpromising for one firm due to a lack of knowledge, although better information would have revealed that an implementation might look mutually promising. 
This result shows the importance of secure industrial symbiosis information sharing frameworks to support firms during the process of ISR evaluation by taking into account ISRs' operational as well as epistemic dimensions.

\subsection{An ISR Opportunity Filtering Case Study}

In this section, we present a case study (adopted from [Andiappan et al., 2016]) to illustrate the applicability of our method and the way our decision support algorithms perform in practice.

\section{6.i Case Description}

The case study that we analyze here consists of three firms active in the Malaysian palm oil industry as one of the key industries in Malaysia's developing economy. The first firm is a Palm Oil Mill (POM) that generates solid biomass waste during the process of palm oil extraction. Although this biomass has the potential to be used for biogas generation, POM (traditionally) discharges this waste. The other two firms in this case study are a firm owning a Biomass-based Tri-generation System (BTS) and a Palm-Based Biorefinery (PBB). The biomass waste (generated by POM) can substitute primary inputs of the other two firms and also can be used directly to establish new production lines (in both PBB and BTS). This shows the potential to establish ISRs among these firms. In particular, POM would be seen as a firm on the provider side of two ISR opportunities with PBB and BTS (as potential resource receivers). Then, all the three firms are interested to learn whether such relations are sufficiently promising to negotiate. E.g., if POM has the potential to reduce its waste discharge cost at a sufficient level, such that $\epsilon_{P O M}$ will be met. 
The potential industrial symbiotic relations between POM-BTS and POM-PBB are the two ISR opportunities that we are aiming to model and analyze using provided values in the case and some reasonable assumptions about missing values. As each ISR can be implemented either as a direct or substitution-based ISR, we will have four ISR settings illustrated in Tables 2.I and 2.2. Note that our focus in this section is to illustrate the applicability of our decision support algorithms and not to analyze the detailed subtleties of the case neither methods for estimating cost values.

In the following, we analyze the case assuming that POM has to discharge $1000 \mathrm{Kg}$ of its biomass waste while PBB and BTS require $1000 \mathrm{Kg}$ and $900 \mathrm{Kg}$ of this waste, respectively.

\subsubsection{ISR Modeling ANd Decision Support Algorithms}

In this case study, the potential to establish ISRs between firms results in a set of ISR opportunities $\Gamma=\left\{\mathcal{I}_{P O M-P B B}, \mathcal{I}_{P O M-B T S}\right\}$. To enable the use of FISOF and $\mathrm{FISOF}^{+}$, we follow Definition I and model these two opportunities as ISR institutions. This is $\mathcal{I}=$ $\left\langle\mathcal{M}, q_{0}, \mathcal{S}_{Q}, \Phi\right\rangle$ for $\mathcal{I} \in \Gamma$ where $\mathcal{M}$ and $q_{0}$ are identical to Example I, $\mathcal{S}_{Q}$ is presented in Tables 2.I and 2.2, and $\Phi$ is the Shapley-based cost sharing mechanism (as formulated in Definition I). Accordingly, in $\mathcal{I}_{P O M-P B B}$, we have that $\Phi\left(P O M, q_{\text {dir }}\right)=\Phi\left(P O M, q_{\text {sub }}\right)=$ $€_{\mathrm{IO} 7.292 \mathrm{II} \text { and }} \Phi\left(P B B, q_{\text {dir }}\right)=\Phi\left(P B B, q_{\text {sub }}\right)=€_{\mathrm{I} 46.392 \mathrm{II} .}$ Moreover, in $\mathcal{I}_{P O M-B T S}$, we have that $\Phi\left(P O M, q_{\text {dir }}\right)=\Phi\left(P O M, q_{\text {sub }}\right)=€_{77.61553}$ and $\Phi\left(B T S, q_{\text {dir }}\right)=\Phi\left(B T S, q_{\text {sub }}\right)$

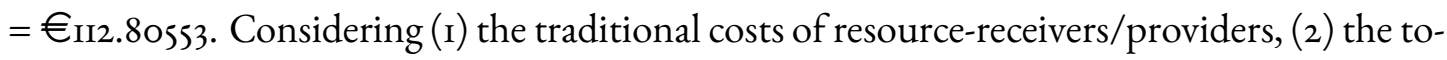
tal costs that firms face with in each of the potential implementations of ISR opportunities in $\Gamma$, and (3) their minimum acceptable cost reductions, we can compute the "excess" cost reduction that firms can enjoy, i.e., $\left(T_{i}-\Phi(i, q)-E_{i}+R_{i}\right)-\epsilon_{i}$. For instance, in $\mathcal{I}_{P O M-B T S}$, 


\begin{tabular}{|c|c|c|}
\hline ISR Settings for $\mathcal{I}_{P O M-P B B}$ & $\mathcal{S}_{\text {dir }}$ & $\mathcal{S}_{\text {sub }}$ \\
\hline Treatment Cost $(€ / \mathrm{Kg})$ & 0.24000 & 0.24000 \\
\hline Transportation Cost $(€ / \mathrm{Kg})$ & 0.00IOo & 0.00I0o \\
\hline Transaction Cost $(€ / \mathrm{Kg})$ & 0.01268 & 0.01268 \\
\hline Biomass Discharge Cost $(€ / \mathrm{Kg})$ & 0.00230 & 0.00230 \\
\hline Biomass Purchase Cost $(€ / \mathrm{Kg})$ & 0.04140 & 0.04140 \\
\hline Incentive $(\mathrm{POM})(€ / \mathrm{Kg})$ & O.IOooo & 0.20000 \\
\hline Incentive $(\mathrm{PBB})(€ / \mathrm{Kg})$ & 0.10000 & 0.20000 \\
\hline Acceptable Reduction $\epsilon_{P O M}(€ / \mathrm{Kg})$ & 0.01522 & 0.01522 \\
\hline Acceptable Reduction $\epsilon_{P B B}(€ / \mathrm{Kg})$ & 0.01522 & 0.01522 \\
\hline POM's Extra Cost $E_{P O M}(€ / \mathrm{Kg})$ & 0.00000 & 0.00000 \\
\hline PBB's Extra Costs $E_{P B B}(€ / \mathrm{Kg})$ & 0.00100 & 0.00000 \\
\hline
\end{tabular}

Table 2.1: ISR Settings for $\mathcal{I}_{P O M-P B B}$ 


\begin{tabular}{|c|c|c|}
\hline ISR Settings for $\mathcal{I}_{P O M-B T S}$ & $\mathcal{S}_{\text {dir }}$ & $\mathcal{S}_{\text {sub }}$ \\
\hline Treatment Cost $(€ / \mathrm{Kg})$ & 0.20000 & 0.20000 \\
\hline Transportation Cost $(€ / \mathrm{Kg})$ & 0.00I0o & o.ooloo \\
\hline Transaction Cost $(€ / \mathrm{Kg})$ & 0.01058 & 0.01058 \\
\hline Biomass Discharge Cost $(€ / \mathrm{Kg})$ & 0.00230 & 0.00230 \\
\hline Biomass Purchase Cost $(€ / \mathrm{Kg})$ & 0.04140 & 0.04140 \\
\hline Incentive $(\mathrm{POM})(€ / \mathrm{Kg})$ & 0.10000 & 0.20000 \\
\hline Incentive (BTS) $(€ / \mathrm{Kg})$ & 0.10000 & 0.20000 \\
\hline Acceptable Reduction $\epsilon_{P O M}(€ / \mathrm{Kg})$ & 0.01269 & 0.01269 \\
\hline Acceptable Reduction $\epsilon_{B T S}(€ / \mathrm{Kg})$ & 0.01269 & 0.01269 \\
\hline POM's Extra Cost $E_{P O M}(€ / \mathrm{Kg})$ & 0.00026 & 0.00026 \\
\hline BTS’s Extra Costs $E_{B T S}(€ / \mathrm{Kg})$ & 0.00560 & 0.00460 \\
\hline
\end{tabular}

Table 2.2: ISR Settings for $\mathcal{I}_{P O M-B T S}$ 
the firm POM can obtain $€_{104.22447}$ which is $€_{92.7992 I}$ above its minimum acceptable cost reduction $\epsilon_{P O M}$ for implementing the substitution-based ISR with BTS (on $900 \mathrm{Kg}$ of biomass). In Tables 2.3 and 2.4 we present the value $v=T_{i}-\Phi(i, q)-E_{i}+R_{i}-\epsilon_{i}$ for each potential implementation and use Definition 3 to determine weather an ISR implementation is a promising one (from a firm's perspective). Figures 2.4, 2.5, and 2.6, display the dynamics of value $v$-as a ranking/evaluation parameter-among all the available ISR implementations for firms POM, PBB, and BTS, respectively.

\begin{tabular}{|c|c|c|}
\hline $\mathcal{I}_{P O M-P B B}$ & $v$ & Promisingness \\
\hline$q_{d i r}$ for POM & $€-20.21316$ & Unpromising for $\operatorname{POM} \boldsymbol{x}$ \\
\hline$q_{d i r}$ for $\mathrm{PBB}$ & $€-2 \mathrm{I} .2 \mathrm{I} 3 \mathrm{I} 6$ & Unpromising for $\mathrm{PBB} \boldsymbol{x}$ \\
\hline$q_{s u b}$ for POM & $€_{79.78684}$ & Promising for POM $\checkmark$ \\
\hline$q_{s u b}$ for $\mathrm{PBB}$ & $€_{79.78684}$ & Promising for PBB $\boldsymbol{}$ \\
\hline
\end{tabular}

Table 2.3: ISR Implementations of $\mathcal{I}_{P O M-P B B}$

To illustrate how our ISR opportunity filtering algorithms perform in practice, here we go through a run of each in this case study and compare their results.

FISOF Algorithm: Using FISOF, firms can learn whether a given ISR is a promising one (Definition 3). In this case study, $\operatorname{FISOF}(P O M, \Gamma)$ returns $\mathcal{I}_{P O M-B T S}$ as the unique promis- 


\begin{tabular}{|c|c|c|}
\hline $\mathcal{I}_{P O M-B T S}$ & $v$ & Promisingness \\
\hline$q_{d i r}$ for POM & $€_{2.79921}$ & Promising for POM $\checkmark$ \\
\hline$q_{d i r}$ for BTS & $€-2.01079$ & Unpromising for BTS $\boldsymbol{x}$ \\
\hline$q_{\text {sub }}$ for POM & $€_{92.79921}$ & Promising for POM $\checkmark$ \\
\hline$q_{\text {sub }}$ for BTS & $€ 88.88921$ & Promising for BTS $\checkmark$ \\
\hline
\end{tabular}

Table 2.4: ISR Implementations of $\mathcal{I}_{P O M-B T S}$

ing ISR for POM. Accordingly, POM rejects $\mathcal{I}_{P O M-P B B}$ due to its epistemic limitations while $\mathrm{PBB}$ and $\mathrm{BTS}$ respectively opt to negotiate $\mathcal{I}_{P O M-P B B}$ and $\mathcal{I}_{P O M-B T S}$ thanks to their epistemic observability. This leads to (in equilibrium) occurrence of ISR negotiation only on $\mathcal{I}_{P O M-B T S}$. Note that although there exists a mutually promising ISR implementation of $\mathcal{I}_{P O M-P B B}$, namely its direct implementation, firms are not able to realize it due to their strategic/epistemic limitations.

FISOF $^{+}$Algorithm: Using FISOF $^{+}$, firms can learn about all the mutually promising implementations of ISR opportunities thanks to information sharing. In this case study, $\mathrm{FISOF}^{+}(P O M, \Gamma)$ returns a ranked set that starts with substitution-based ISR with BTS (as the most promising implementation for POM) and ends with substitution-based ISR with PBB (as the least promising implementation for POM). Note that although the direct ISR with BTS is a promis- 
ing implementation for POM, it is not among the generated outputs of $\mathrm{FISOF}^{+}$because it is not mutually promising for both sides of the relation. By applying $\mathrm{FISOF}^{+}$, we also have that $\mathrm{FISOF}^{+}(P B B, \Gamma)$ and $\mathrm{FISOF}^{+}(B T S, \Gamma)$ both return substitution-based ISR with POM. Accordingly, we have that thanks to the fostering effect of information sharing, the ISR negotiations on all the mutually promising implementations occur in equilibrium.

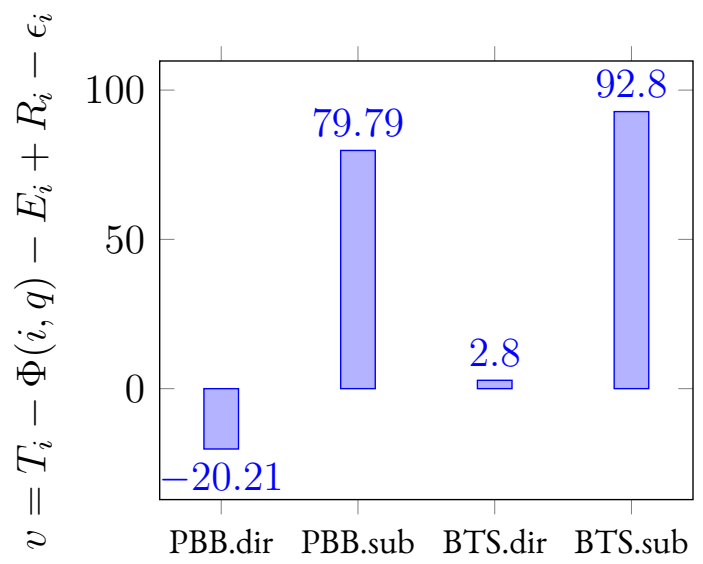

Figure 2.4: $v$ Value for POM's Potential ISR Implementations.

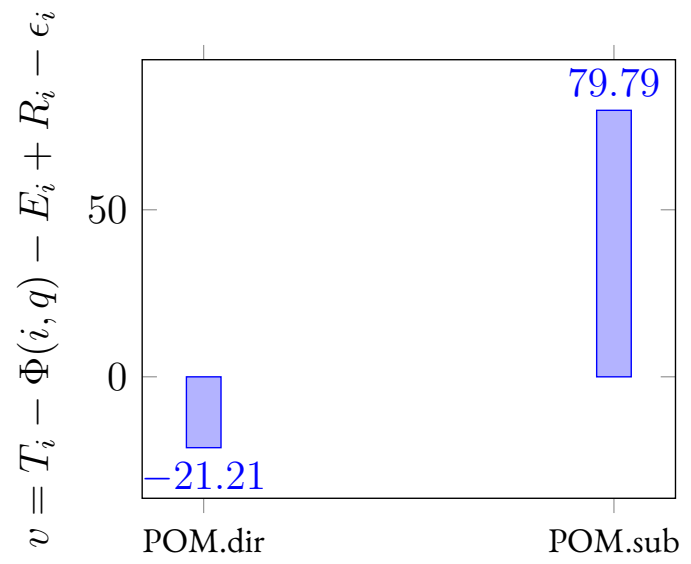

Figure 2.5: $v$ Value for PBB's Potential ISR Implementations. 


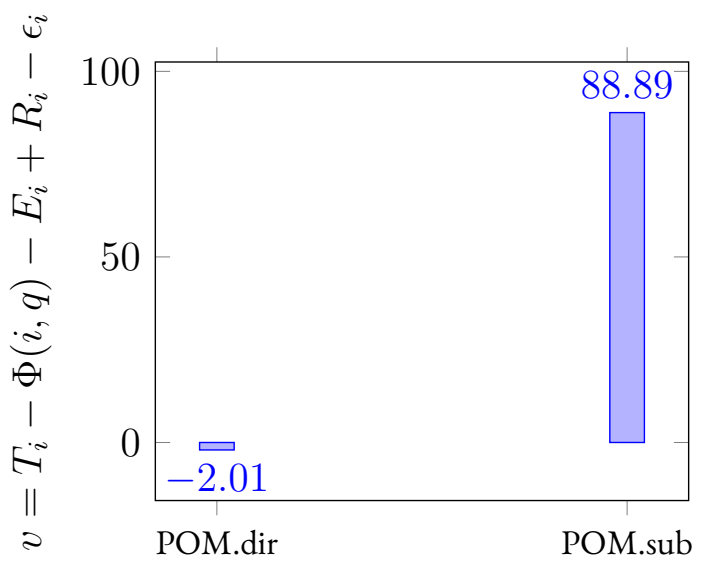

Figure 2.6: $v$ Value for BTS's Potential ISR Implementations.

As illustrated in this case study and following what we formally evaluated in Section 2.5.2, information sharing enables the use of $\mathrm{FISOF}^{+}$and accordingly the chance to negotiate all the mutually promising ISR implementations (that may be dismissed under FISOF). We highlight that dismissing mutually promising implementations may avoid the occurrence of negotiations to form Industrial Symbiotic Networks (ISNs) [Yazdanpanah et al., 2018]. For instance, in our case study, POM may opt to implement ISRs with both PBB and BTS. While FISOF avoids such ISN negotiations, $\mathrm{FISOF}^{+}$enables it as it relies on collectively available strategies under distributed knowledge.

\subsection{Concluding Remarks}

In this section, we highlight different aspects of our contribution, present its applicability domains, briefly discuss the validity of presented ISR dimensions, and conclude with further research directions. 
Contributions and Applicability: In this chapter, we presented a formal decision support method that takes into account operational and epistemic aspects of ISRs for filtering industrial symbiosis opportunities (FISOF). Using this method, firms can evaluate any particular ISR opportunity with respect to obtainable cost reductions under some observable implementations of the opportunity. This in turn enables ranking the set of promising ISR opportunities for a firm. Accordingly, firms learn about a spectrum that begins with the most-promising and ends with least-promising ISR opportunities. Then, a firm may develop strategies how to pursue the contract negotiations with members of this spectrum and dismisses other (unpromising) ISR opportunities. This results in a quantitative operationsoriented decision support algorithm for ISR evaluation. Moreover, we show that firms' epistemic limitations may prohibit the learning about all the mutually promising ISR implementations. As a remedy, we developed a method $\left(\mathrm{FISOF}^{+}\right)$that employs firms' distributed knowledge and enables learning about all the mutually promising ISR implementations (that may be dismissed if firms opt not to share information). In addition to the algorithmic account of these two methods, we introduced new operational semantics for industrial symbiosis research and presented novel concepts for reasoning about ISRs. Such operational semantics enable systematic reasoning about ISR behavior and foster the computation of ISR properties (e.g. by employing multiagent-based simulation methods [Yazan et al., 2017b]).

The other application of our work is to support policy-making and fine-tuning regarding regulations that foster the transition to the circular economy. For instance, due to a lack of regulations, firms may face no prohibition on disposal of some particular (hazardous) wastes or may receive no incentives in case of substituting some of their raw material with reusable waste inputs. Using our method, policy-makers can analyze ISRs towards which they can aim 
their encouraging incentives or binding regulations. Moreover, policy-makers can learn if by means of modifications in regulations or/and incentives, an unpromising ISR implementation can turn to a promising one (if-then analysis).

VAlidity of ISR Dimensions and Algorithms: The presented ISR dimensions (Figures 2.I and 2.2) and the ISR 3 T operational costs, i.e. transportation, treatment, and transaction costs, are supported by industrial symbiosis research literature (see Section 2.2). In addition, to back-up the theory with expert knowledge, we circulated an earlier version of this work among our industrial partners in the EU-funded $S H A R E B O X$ project [SHAREBOX, 2019], presented the framework as well as various implementations of FISOF and $\mathrm{FISOF}^{+}$to their representatives in validation sessions, and have taken into account their practice-oriented feedback.

FUTURE WORK: We illustrated that negotiations on an ISR implementation that is not mutually promising do not occur in an equilibrium even if it is a desirable one (e.g., from an environmental point of view). Then, as we discussed earlier, policy-makers can learn about symbiotic relations that are not implementable and introduce monetary incentives to foster them. This leads to the so called incentive engineering problem. Roughly speaking, "how a limited amount of incentive can be optimally distributed among unpromising ISRs such that the outcome meets a policy" would be a question that calls for policy support tools. As an extension, we plan to address this problem using computational methods for incentive allocation [Wooldridge et al., 2013] and auction mechanisms in multi-agent systems [Vulkan and Jennings, 2000].

In this work, we presented ISR opportunity evaluation methods-usable as pre-negotiation 
decision support tools. In future work, we plan to develop an automated procedure that acts as a middle-ware in between $\mathrm{FISOF}^{+}$and established negotiation methods. For such a purpose, we aim to build on multiagent-based implementations of Delphi [García-Magariño et al., 2008] and agent-based methods [García-Magariño, 2013] that enable representing potential transformations of ISR institutions.

We also aim to extend our methods and operational semantics to capture symbiotic relations with more players and evaluate Industrial Symbiotic Networks (ISNs) [Yazdanpanah et al., 2016]. We emphasize that structural properties and dynamics of power relations in ISNs result in more complex institutional behaviors, hence calls for (I) representations that take into account the symbiotic network as a coordinated institution [Yazdanpanah et al., 2018] and (2) dynamic contracts able to monitor and enforce the commitment of involved firms to the codes of conduct [Dastani et al., 2017b]. 

The market is no god, it cannot solve every problem.

Eric Maskin

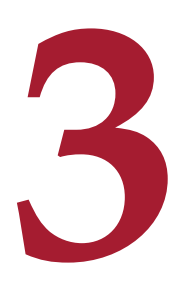

\section{Coordinating Industrial Symbiosis}

Networks

WE PRESENT A COORDINATED MULTIAGENT FRAMEWORK for implementing a class of collaborative industrial practices called "Industrial Symbiotic Networks (ISNs)" as cooperative 
games. The game-theoretic formulation of collaboration in ISNs enables systematic reasoning about what we call the ISN implementation problem. Specifically, the characteristics of ISNs may lead to inapplicability of fair and stable benefit allocation methods. Inspired by realistic ISN scenarios and following the literature on normative multiagent systems, we consider regulations and normative socioeconomic policies as coordination instruments that in combination with ISN games resolve the situation ${ }^{\mathrm{I}}$. In this multiagent system, employing Marginal Contribution Nets (MC-Nets) as rule-based cooperative game representations fosters the combination of regulations and ISN games with no loss in expressiveness. We develop algorithmic methods for generating regulations that ensure the implementability of ISNs and as a policy support, present the policy requirements that guarantee the implementability of all the desired ISNs in a balanced-budget way.

\section{I INTRODUCTION}

Industrial Symbiotic Networks (ISNs) are collaborative networks of industries with the aim to reduce the use of virgin material by circulating reusable resources (e.g, physical waste material and energy) among the network members [Chertow, 200o; Lombardi and Laybourn, 20I2; Yazan et al., 2016]. In such networks, symbiosis leads to socioeconomic and environmental benefits for involved industrial agents and the society. One barrier against stable ISN implementations is the lack of frameworks able to secure such networks against unfair and unstable allocation of obtainable benefits among the involved industrial firms. In other words, although in general ISNs result in a reduction of the total cost, a remaining

${ }^{\text {IT }}$ The content of this chapter is mainly based on "Vahid Yazdanpanah, Devrim Murat Yazan, and W. Henk M. Zijm. Coordinated multiagent industrial symbiosis systems. Automatica, pages (under-review), 2019c" [Yazdanpanah et al., 2019c]. 
challenge for operationalization of ISNs is to tailor reasonable mechanisms for allocating the total obtainable cost reductions - in a fair and stable manner-among the contributing firms. Otherwise, even if economic benefits are foreseeable, a lack of stability and/or fairness may lead to non-cooperative decisions. This will be the main focus of what we call the industrial symbiosis implementation problem. Reviewing recent contributions in the field of industrial symbiosis research, we encounter studies focusing on the necessity to consider interrelations between industrial enterprises [Yazan et al., 2016; Yazdanpanah et al., 2019e] and the role of contract settings in the process of ISN implementation [Albino et al., 2016]. We believe that a missed element for shifting from theoretical ISN design to practical ISN implementation is to model, reason about, and support ISN decision processes in a dynamic way (and not by using snapshot-based modeling frameworks).

For such a multiagent setting, the mature field of cooperative game theory provides rigorous methodologies and established solution concepts, e.g. the core of the game and the Shapley allocation [Borm et al., 20oI; Driessen, 2013; Mas-Colell et al., 1995; Osborne and Rubinstein, 1994]. However, for ISNs modeled as a cooperative game, these established solution concepts may be either non-feasible (due to properties of the game, e.g. being unbalanced) or non-applicable (due to properties that the industrial domain asks for but solution concepts cannot ensure, e.g. individual as well as collective rationality). This calls for contextualized multiagent solutions that take into account both the complexities of ISNs and the characteristics of the employable game-theoretical solution concepts. Accordingly, inspired by realistic ISN scenarios and following the literature on normative multi-agent systems [Andrighetto et al., 2013; Grossi et al., 2013; Shoham and Tennenholtz, 1995], we consider regulative rules and normative socioeconomic policies as two elements that in combination with 
ISN games result in the introduction of the novel concept of Coordinated ISNs $(\mathcal{C}-I S N s)^{2}$. We formally present regulations as monetary incentive rules to enforce desired industrial collaborations with respect to an established policy. Regarding our representational approach, we use Marginal Contribution Nets (MC-Nets) as rule-based cooperative game representations. This simply fosters the combination of regulative rules and ISN games with no loss in expressiveness. Accordingly, applying regulatory rules to ISNs enables ISN policy-makers to transform ISN games and ensures the implementability of desired ones in a fair and stable manner.

In this work, we provide a coordinated multiagent system - using MC-net cooperative games-for the implementation phase of ISNs. Moreover, we develop algorithmic methods for generating regulations that ensure the implementability of ISNs. Finally, as a policy support, we discuss the ISN policy requirements that guarantee the implementability of all the desired industrial collaborations in a balanced-budget way.

This chapter is structured as follows. Section 3.2 provides a conceptual analysis on ISNs and allocation problems in such multiagent collaborative networks. Section 3.3 introduces preliminary formal notions and game theoretic solution concepts required for our ISN implementation framework. Sections 3.4 and 3.5 present our ISN frameworks and illustrate the verified results on effectivity of the developed coordinated multiagent system for implementing industrial symbiosis. Finally, Section 3.6 concludes the chapter by highlighting the main contributions and potential extensions of this work.

\footnotetext{
${ }^{2}$ See [Wooldridge, 2009] for multiagent solution concepts in general and [Bussmann et al., 20I3; Jennings, 1994] for their application in the industrial domain.
} 


\subsection{Conceptual Analysis}

In this section, we (I) present the intuition behind our approach using a running example, (2) discuss our norm-based perspective for capturing ISN regulations, (3) describe the evaluation criteria for an ideal ISN implementation framework, and 4) review previous work on tailoring game-theoretic solution concepts for the industrial symbiosis implementation problem.

\subsection{ISN as a Multiagent Practice}

To explain the dynamics of implementing ISNs as multiagent industrial practices, we use a running example. Imagine three industries $i, j$, and $k$ in an industrial park such that $r_{i}, r_{j}$, and $r_{k}$ are among recyclable resources in the three firms' wastes, respectively. Moreover, $i, j$, and $k$ require $r_{k}, r_{i}$, and $r_{j}$ as their primary inputs, respectively. In such scenarios, discharging wastes and purchasing traditional primary inputs are transactions that incur cost. Hence, having the chance to reuse a material, firms prefer recycling and transporting reusable resources to other enterprises if such transactions result in obtainable cost reductions for both parties-meaning that it reduces the related costs for discharging wastes (on the resource provider side) and purchasing cost (on the resource receiver side). On the other hand, the implementation of such an industrial network involves transportation, treatment, and transaction costs. In principle, aggregating resource treatment processes using refineries, combining transaction costs, and coordinating joint transportation may lead to significant cost reductions at the collective level.

What we call the industrial symbiosis implementation problem focuses on challengesand accordingly seeks solutions-for sharing this collectively obtainable benefit among the involved firms. Simply stated, the applied method for distributing the total obtainable ben- 
efit among involved agents is crucial while reasoning about implementing an ISN.

Example 3 (Running Example). Imagine a scenario in which symbiotic relations $i j, i k$, and $j k$, respectively result in 4,5 , and 4 utility units of benefit, the symbiotic network $i j k$ leads to 6 units of benefit, and each agent can be involved in at most one symbiotic relation. To implement the $i j k$ ISN, one main question is about the method for distributing the benefit value 6 among the three agents such that they all be induced to implement this ISN. For instance, as $i$ and $k$ can obtain 5 utils together, they will defect the ISN $i j k$ if we divide the 6 units of util equally ( 2 utils to each agent). We return to this example through the course of this chapter.

Note that allocating benefit values lower than their "traditional" benefits-that is obtainable in case firms defect the collaboration-results in unstable ISNs. Moreover, unfair mechanisms that disregard the contribution of firms may cause the firms to move to other ISNs that do so. In brief, even if an ISN results in sufficient cost reductions (at the collective level), its implementation and applied allocation methods determine whether it will be realized and maintained. Our main objective in this work is to provide a multiagent implementation framework for ISNs that enables fair and stable allocation of obtainable benefits. In further sections, we review two standard allocation methods, discuss their applicability for benefit-sharing in ISNs, and introduce our normatively-coordinated multiagent system to guarantee stability and fairness in ISNs.

\subsubsection{ISN Regulations as Socioeconomic Norms}

In real cases, ISNs take place under regulations that concern environmental as well as societal policies. Hence, industrial agents have to comply to a set of rules. For instance, avoiding waste 
discharge may be encouraged (i.e., normatively promoted) by the local authority or transporting a specific type of hazardous waste may be forbidden (i.e., normatively prohibited) in a region. Accordingly, to nudge the collective behavior, monetary incentives in the form of subsidies and taxes are well-established solutions. This shows that the ISN implementation problem is not only about decision processes among strategic utility-maximizing industry representatives (at a microeconomic level) but in addition involves regulatory dimensionssuch as the presence of binding/encouraging monetary incentives (at a macroeconomic level).

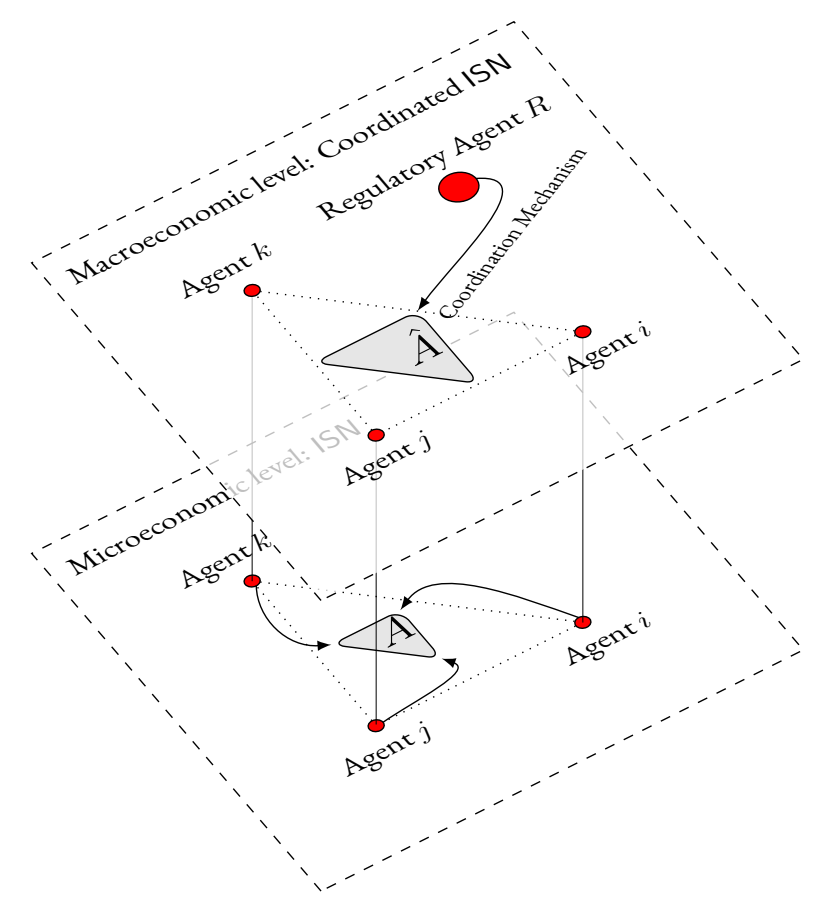

Figure 3.1: At the microeconomic level, $A$ represents the set of all benefit allocation methods that are preferable for all the firms. At the macroeconomic level, due to the introduced coordination mechanism by the regulatory agent (respecting the established socioeconomic policy), we have the allocation set $\hat{A}$ either equal to $A$ or as a shrunk/extended version of it.

To capture the regulatory dimension of ISNs, we apply a normative policy that respects the socioeconomic as well as environmental desirables and categorizes possible coalitions of 
industries in three classes of: promoted, permitted, and prohibited. Accordingly, the regulatory agent respects this classification and allocates incentives such that industrial agents will be induced to: implement promoted ISNs and avoid prohibited ones (while permitted ISNs are neutral from the policy-maker's point of view). For instance, in our ISN scenario, allocating 10 units of incentive to $i j k$ and 0 to other possible ISNs induces all the rational agents to form the grand coalition and implement $i j k$-as they cannot benefit more in case they defect. We call ISNs that take place under regulations, Coordinated ISNs $(\mathcal{C}-I S N s)$. Note that the term "coordination" in this context refers to the application and efficacy of monetary incentive mechanisms in the ISN implementation phase, and should not be confused with ISN administration (i.e., managing the evolution of relations). Figure 3.I presents a schematic view on the role of the regulatory agents in $\mathcal{C}-$ ISNs.

\subsubsection{Evaluation Criteria For ISN IMPLEMENTATION Frameworks}

Dealing with firms that perform in a complex multiagent industrial context calls for implementation platforms that can be tuned to specific settings, can be scaled for implementing various ISN topologies, do not require industries to sacrifice financially, and allow industries to practice their freedom in the market. We deem that the quality of an ISN implementation framework should be evaluated by (I) Generality as the level of flexibility in the sense of its independence from agents' internal reasoning processes (i.e., how much the framework adheres to the principle of separation of concerns), (2) Expressivity as the level of scalability in the sense of its independence from size and topology of the network, (3) Rationality as the level of compliance of the employed allocation mechanisms to the collective as well as individual rationality axiom (i.e., no agent (group) participates in a cooperative practice if 
they expect higher utility otherwise), and (4) Autonomy as the level of allowance (i.e., nonrestrictiveness) of the employed coordination mechanisms. Then an ideal framework for implementing ISNs should be general-i.e., it should allow for manipulation in the sense that the network designer does not face any re-engineering/calibration burden-sufficiently expressive, rationally acceptable for all firms, and respect their autonomy. The goal of this chapter is to develop an implementation framework for ISNs that has properties close to the ideal one.

\subsubsection{Previous Work at a Glance}

The idea of employing cooperative game theory for analysis and implementation of industrial symbiosis have only been sparsely explored [Chew et al., 2009; Grimes-Casey et al., 2007; Yazdanpanah and Yazan, 2017]. In [Grimes-Casey et al., 2007], the authors use both cooperative and non-cooperative game theory for analyzing the behavior of firms engaged in a case-specific industrial ecology. While the analysis is expressive, the implemented relations are specific to refillable/disposable bottle life cycles. In [Chew et al., 2009], the authors tailor a mechanism for allocating costs among participating agents that expects an involved industry to "bear the extra cost". Although such an approach results in collective benefits, it is not in-line with the individual rationality axiom. In [Yazdanpanah and Yazan, 20I7] bilateral industrial symbiotic relations are modeled as cooperative games; the authors show that in such a specific class, with only two industries involved, the total operational costs can be allocated fairly and stably. In this chapter, we relax the limitation on the number of involved industries and use the concept of Marginal Contribution Nets (MC-Nets), which enables a representation that is sufficiently expressive to capture the regulatory aspect of ISNs. We will give a more 
detailed review of previous work in Section 3.3.2 after covering the technical background.

\subsection{Preliminaries}

In this section, we recall the preliminary notions in cooperative games, the MC-Net representation of such games, and the two principal solution concepts: the Shapley value and the Core $^{3}$. Moreover, we discuss in more detail the technical aspects of previous work that applied game-theoretical methods for ISN modeling and analysis.

\subsection{TeCHNicAl BaCKground}

In this work, we build on the transferable utility assumption in multiagent settings. This is to assume that the payoff to a group of agents involved in an ISN (as a cooperative practice) can be freely distributed among group members.

Cooperative Games: Multiagent cooperative games with transferable utility are often modeled by the tuple $(N, v)$, where $N$ is the finite set of agents and $v: 2^{N} \mapsto \mathbb{R}$ is the characteristic function that maps each possible agent group $S \subseteq N$ to a real-valued payoff $v(S)$. In such games, the so-called allocation problem focuses on methods to distribute $v(S)$ among all the agents (in $S$ ) in a reasonable manner. That is, $v(S)$ is the result of a potential cooperative practice, hence ought to be distributed among agents in $S$ such that they all be induced to cooperate (or remain in the cooperation). Various solution concepts specify the utility each agent receives by taking into account properties like fairness and stability. The two standard solution concepts that characterize fair and stable allocation of benefits are the Shapley value and the Core, respectively.

\footnotetext{
${ }^{3}$ The presented material on basics in cooperative games is based on [Mas-Colell et al., 1995; Osborne and Rubinstein, 1994] while for the MC-Net notations, we build on [Ieong and Shoham, 2005; Lesca et al., 2017].
} 
Shapley Value: The Shapley value prescribes a notion of fairness. It says that assuming the formation of the grand coalition $N=\{1, \ldots, n\}$, each agent $i \in N$ should receive its average marginal contribution over all possible permutations of the agent groups. Let $s$ and $n$, represent the cardinality of $S$ and $N$, respectively. Then, the Shapley value of $i$ under characteristic function $v$, denoted by $\Phi_{i}(v)$, is formally specified as $\Phi_{i}(v)=\sum_{S \subseteq N \backslash\{i\}} \frac{s !(n-s-1) !}{n !}(v(S \cup$ $\{i\})-v(S))$. For a game $(N, v)$, the unique list of real-valued payoffs $x=\left(\Phi_{1}(v), \cdots, \Phi_{n}(v)\right) \in$ $\mathbb{R}^{n}$ is called the Shapley allocation for the game. The Shapley allocation have been extensively studied in the game theory literature and satisfies various desired properties in multi-agent practices. Moreover, it can be axiomatized using the following properties.

- Efficiency (EFF): The overall available utility $v(N)$ is allocated to the agents in $N$, i.e., $\sum_{i \in N} \Phi_{i}(v)=v(N)$.

- Symmetry (SYM): Any arbitrary agents $i$ and $j$ that make the same contribution receive the same payoff, i.e., $\Phi_{i}(v)=\Phi_{j}(v)$.

- Dummy Player (DUM): Any arbitrary agent $i$ of which its marginal contribution to each group $S$ is the same, receives the payoff that it can earn on its own; i.e., $\Phi_{i}(v)=v(\{i\})$.

- Additivity (ADD): For any two cooperative games $(N, v)$ and $(N, w), \Phi_{i}(u+w)=$ $\Phi_{i}(v)+\Phi_{i}(w)$ for all $i \in N$, where for all $S \subseteq N$, the characteristic function $v+w$ is defined as $(v+w)(S)=v(S)+w(S)$.

In the following, we refer to an allocation that satisfies these properties as a fair allocation. Core of the Game: In core allocations, the focus is on the notion of stability. In brief, an allocation is stable if no agent (group) benefits by defecting the cooperation. Formally, for a 
game $(N, v)$, any list of real-valued payoffs $x \in \mathbb{R}^{n}$ that satisfies the following conditions is a core allocation for the game:

- Rationality (RAT): $\forall S \subseteq N: \sum_{i \in S} x_{i} \geq v(S)$

- Efficiency (EFF): $\sum_{i \in N} x_{i}=v(N)$

One main question is whether for a given game, the core is non-empty (i.e., whether there exists a stable allocation for the game). A game for which there exist a non-empty set of stable allocations should satisfy the balancedness property, defined as follows. Let $1_{S} \in \mathbb{R}^{n}$ be the membership vector of $S$, where $\left(1_{S}\right)_{i}=1$ if $i \in S$ and $\left(1_{S}\right)_{i}=0$ otherwise. Moreover, let $\left(\lambda_{S}\right)_{S \subseteq N}$ be a vector of weights $\lambda_{S} \in[0,1]$. A vector $\left(\lambda_{S}\right)_{S \subseteq N}$ is a balanced vector if for all $i \in N$, we have that $\sum_{S \subseteq N} \lambda_{S}\left(1_{S}\right)_{i}=1$. Finally, a game is balanced if for all balanced vectors of weights, we have that $\sum_{S \subseteq N} \lambda_{S} v(S) \leq v(N)$. Finally, based on the standard results in [Bondareva, 1963; Shapley, 1967] (the Bondereva-Shapley theorem), we have that a game has a non-empty core if and only if it is balanced.

In the following, we refer to an allocation that satisfies RAT and EFF as a stable allocation. Marginal Contribution Nets (MC-Nets): Representing cooperative games by their characteristic functions (i.e., specifying values $v(S)$ for all the possible coalitions $S \subseteq N$ ) may become unfeasible in large-scale applications. In this work, as we are aiming to implement ISNs in a scalable manner, we employ a basic MC-Net [Ieong and Shoham, 2005] representation that uses a set of rules to specify the value of possible agent coalitions. Moreover, as we wish to capture the regulatory aspect of ISNs, employing rule-based game representations appears to be a natural approach. 
Definition 4 (Basic MC-Net [Ieong and Shoham, 2005] ). A basic MC-Netrepresents the cooperative game among agents in $N$ as a finite set of rules $\left\{\rho_{i}:\left(\mathcal{P}_{i}, \mathcal{N}_{i}\right) \mapsto v_{i}\right\}_{i \in K}$, where $\mathcal{P}_{i} \subseteq N, \mathcal{N}_{i} \subset N, \mathcal{P}_{i} \cap \mathcal{N}_{i}=\emptyset, v_{i} \in \mathbb{R} \backslash\{0\}$, and $K$ is the set of rule indices. For an agent coalition $S \subseteq N$, a rule $\rho_{i}$ is applicable if $\mathcal{P}_{i} \subseteq S$ and $\mathcal{N}_{i} \cap S=\emptyset$ (i.e., $S$ contains all the agents in $\mathcal{P}_{i}$ and no agent in $\left.\mathcal{N}_{i}\right)$. Let $\Pi(S)$ denote the set of rule indices that are applicable to $S$. Then for the value of $S$, denoted by $v(S)$, we have that $v(S)=\sum_{i \in \Pi(S)} v_{i}$.

In further sections, we present an MC-Net representation of the $i j k$ ISN scenario and illustrate how this rule-based representation enables applying norm-based coordination to ISNs.

\subsubsection{Revisiting Previous Work}

Chew et al. analyze the interaction of participating companies in an Eco-industrial park seeking to develop a game-theoretic implementation framework for inter-plant water integration [Chew et al., 2009]. In their cooperative game model, by assuming the compliance of agents to their commitments, the optimum collective benefit is achievable. As the authors mention, in case the cooperation takes place, their allocation mechanism results in higher collective payoff in comparison to their non-cooperative game scheme. This result is achieved through adding contextualized interaction protocols that compel the industries to act in a desired manner. Roughly speaking, it is assumed that the network manager has control over internal operations and decision processes of involved agents (which may be applicable in specific case studies but is in contrast with the principle of separation of concerns). For instance, given the availability of an optimal wastewater interchange scheme, it is shown that in case the agents adopt the scheme and act accordingly, they can benefit both individually and 
collectively. In other words, the focus is shifted towards providing methods for optimizing the scheme in a specific case.

In a more recent work, Yazdanpanah and Yazan study the modeling and implementation of industrial symbiotic relations as two person cooperative games [Yazdanpanah and Yazan, 2017]. Their focus is on the allocation of the total operational cost among involved agents using a tailored version of the Shapley value and the standard notion of core. They show that for industrial symbiotic relation games, core is non-empty and hence such symbiotic practices are implementable in a stable manner. Moreover, as the Shapley value will be in the core, it is rational for industries to implement the Shapley allocation (with no need for interruption by the regulatory agent). Notice that although their industrial symbiosis implementation satisfies desired properties, e.g., autonomy and rationality, it is not expressive for implementing symbiotic relations among three or more industries. This is basically because their analysis is based on properties of two-person games.

Finally, Grimes-Casey et al. [Grimes-Casey et al., 2007] focus on cooperative decisionmaking and heterogeneity of the involved agents (with respect to their epistemic states) in an industrial symbiosis scenario. They employ cost-based mechanisms to nudge the behavior of manufacturer as well as consumer agents towards using refillable beverage containers. Although their cooperative management framework is problem-specific, it is expressive and scalable as they employ profit values that are computable in low complexity. They also state that in real cases the applicability of most cooperative game solution concepts depends on government enforcement. This is in-line with our attempt to capture the regulatory aspect of industrial symbiosis using incentive mechanisms. 


\subsection{ISN GAMES}

As discussed in [Albino et al., 2016; Yazdanpanah and Yazan, 20I7], the total obtainable cost reduction — as the economic benefit — and its allocation among involved firms are key drivers behind the stability of ISNs. For any set of industrial agents $S$, this total value can be computed based on the total traditional cost, denoted by $T(S)$, and the total ISN operational cost, denoted by $O(S)$. In brief, $T(S)$ is the summation of all the costs that firms have to pay in case the ISN does not occur (i.e., to discharge wastes and to purchase traditional primary inputs). On the other hand, $O(S)$ is the summation of costs that firms have to pay collectively in case the ISN is realized (i.e., the costs for recycling and treatment, for transporting resources among firms, and finally the transaction costs). Accordingly, for a non-empty finite set of industrial agents $S$ the obtainable symbiotic value $v(S)$ is equal to $T(S)-O(S)$. In this work, we assume a potential ISN, with a positive total obtainable value, and aim for tailoring gametheoretic value allocation and accordingly coordination mechanisms that guarantee a fair and stable implementation of the symbiosis.

\subsection{ISNs as Cooperative Games}

Our ijk ISN scenario, see Example 3, can be modeled as a cooperative game in which $v(S)$ for any empty/singleton $S$ is 0 and agent groups $i j, i k, j k$, and $i j k$ have the values $4,5,4$, and 6 , respectively. Note that as the focus of ISNs are on the benefit values obtainable due to potential cost reductions, all the empty and singleton agent groups have a zero value because cost reduction is meaningless in such cases. In the game theory language, the payoffs in ISN 
games are normalized. Moreover, the game is superadditive 4 in nature (basically because any firm will only join a coalition if there is something to win, both for that firm and for the others). So, given the traditional and operational cost values for all the possible agent groups $S$ (i.e., $T(S)$ and $O(S))$ in the non-empty finite set of industrial agents $N$, the ISN among agents in $N$ can be formally modeled as follows.

Definition 5 (ISN Games). Let $N$ be a non-empty finite set of industrial agents. Moreover, for any agent group $S \subseteq N$, let $T(S)$ and $O(S)$ respectively denote the total traditional and operational costs for $S$. We say the ISN among industrial agents in $N$ is a normalized superadditive cooperative game $(N, v)$ where $v(S)$ is:

$$
v(S)= \begin{cases}0, & \text { if }|S| \leq 1 \\ T(S)-O(S), & \text { otherwise }\end{cases}
$$

According to the following proposition, basic MC-Nets can be used to represent ISNs. In further sections, this representation aids combining ISN games with normative coordination rules.

Proposition 3 (ISNs as MC-Nets). Any ISN can be represented as a basic MC-Net.

Proof. We provide a constructive proof by (I) introducing an algorithm for specification of all the required MC-Net rules and (2) showing that the constructed MC-Net is equal to the original ISN game. $(I)$ - Let $(N, v)$ be an arbitrary ISN game among industrial agents in $N$.

${ }^{4} \mathrm{~A}$ game is superadditive when the value of the union of two disjoint sets of agents is no less than the sum of the values of the individual sets. In the context of industrial symbiosis, superadditivity implies that forming a symbiotic coalition of industrial agents either results in no value or in a positive value. Implicitly, growth of a group can never result in decrease of the value. 
Moreover, let $S_{\geq 2}=\{S \subseteq N:|S| \geq 2\}$ be the set of all agent groups with two or more members and let $K=\left|S_{\geq 2}\right|$ denote its cardinality. We start with an empty set of rules. Then for all agent groups $S_{i} \in S_{\geq 2}$, for $i=1, \ldots, K$, we add a rule $\left\{\rho_{i}:\left(S_{i}, N \backslash S_{i}\right) \mapsto\right.$ $\left.v_{i}=T\left(S_{i}\right)-O\left(S_{i}\right)\right\} \cdot(2)$ - As in all the constructed rules $\rho_{i}$ it holds that $\mathcal{P}_{i} \cap \mathcal{N}_{i}=\emptyset$ and $\mathcal{P}_{i} \cup \mathcal{N}_{i}=N$, we have that $\sum_{i \in \Pi(S)} v_{i}$ is equal to $v(S)$ for all the members of $S_{\geq 2}$. Moreover, $\Pi(S)$ for empty and singleton agent groups would be empty, hence reflects the 0 value for such groups in the original game.

Note that the proof does not simply rely on the representation power and expressivity of MC-Nets (as shown in [Ieong and Shoham, 2005]) but provides a constructive method that respects the context of industrial symbiosis and related cost values to generate all the required rules for representing ISNs as MC-Nets.

Example 4 (ISN Scenario). Our running example can be represented by the basic MC-Net ${ }^{5}$ $\left\{\rho_{1}:(i j, k) \mapsto 4, \rho_{2}:(i k, j) \mapsto 5, \rho_{3}:(j k, i) \mapsto 4, \rho_{4}:(i j k, \emptyset) \mapsto 6\right\}$

\subsubsection{Benefit Allocation Mechanisms and ISN Games}

As discussed earlier, how firms share the obtainable ISN benefits plays a key role in the process of ISN implementation, mainly due to stability and fairness concerns. Roughly speaking, industrial firms are economically rational firms that defect non-beneficial relations (instability) and mostly tend to reject ISN proposals in which benefits are not shared with respect to their contribution (unfairness). In this work, we focus on Core- and Shapley-allocation mechanisms as two standard methods that characterize stability and fairness in cooperative games, respectively. We show that these solution concepts are applicable in a specific class of ISNs

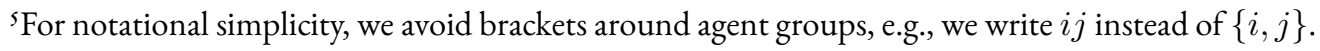


but are not generally scalable for value allocation in the implementation phase of ISNs. This motivates introducing incentive mechanisms to guarantee the implementability of "desired" ISNs.

\section{Two-Person Industrial Symbiosis Games}

When the game is between two industrial firms (i.e., a bilateral relation between a resource receiver/provider couple), it has additional properties that result in applicability of both Core and Shapley allocations. We denote the class of such ISN games by $\operatorname{ISN}_{\Lambda}$. This is, $\operatorname{ISN}_{\Lambda}=$ $\{(N, v):(N, v)$ is an ISN game and $|N|=2\}$. The class of ISN games with three or more agents is denoted by ISN . The class of ISN $_{\Lambda}$ games corresponds to the so called ISR games in [Yazdanpanah and Yazan, 2017]. The difference is on the value allocation perspective as in [Yazdanpanah and Yazan, 2017], the authors assume the elimination of traditional costs (thanks to the implementation of a symbiotic relation) and focus on the allocation of operational costs; while we focus on the allocation of the total benefit, obtainable due to potential cost reductions.

Lemma I (ISN $\mathbf{\Lambda}_{\mathbf{\Lambda}}$ Balancedness). Let $(N, v)$ be an arbitrary ISN $_{\Lambda}$ game. It always holds that $(N, v)$ is balanced.

Proof. Based on the line of argument in [Driessen, 2013; Shapley, 1971] we show that any $\mathrm{ISN}_{\Lambda}$ game is supermodular which directly implies balancedness. For supermodularity, we need to show that, in $(N, v)$, for any couple of arbitrary agent groups $S, T \subseteq N$, we have $v(S)+v(T) \leq v(S \cup T)+v(S \cap T)$. In ISN $_{\Lambda}$ games, by checking the validity of this

inequality for all the six possible $S, T$ combinations, the claim will be proved. For $S=\emptyset$, we have the following valid inequality $v(\emptyset)+v(T) \leq v(\emptyset \cup T=T)+v(\emptyset)$. For $S=N$, 
the inequality can be reformulated in the following valid form $v(N)+v(T) \leq v(N \cup T=$ $N)+v(N \cap T=T)$. Finally, when $S$ and $T$ are equal to the only possible (disjoint) singleton groups, we have $v(S)+v(T) \leq v(N)+v(\emptyset)$ which holds thanks to the superadditivity of ISN games (see an alternative proof in Appendix B).

Relying on Lemma I, we have the following result that focuses on the class of $\mathrm{ISN}_{\Lambda}$ relations and shows the applicability of two standard game-theoretic solution concepts for implementing fair and stable industrial symbiotic networks.

Theorem 3 (Fair and Stable $\mathbf{I S N}_{\Lambda}$ Games). Let $(N, v)$ be an arbitrary ISN $_{\Lambda}$ game. The symbiotic relation among industrial agents in $N$ is implementable in a unique stable and fair manner.

Proof. Stability: As discussed earlier, core allocations guarantee the stability conditions (i.e., RAT and EFF). However, the core is only an applicable solution concept for balanced games. According to Lemma I, we have that $\mathrm{ISN}_{\Lambda}$ games are balanced. Hence, the core of any arbitrary $\mathrm{ISN}_{\Lambda}$ game is nonempty and any allocation in the core guarantees the stability. Stability and Fairness: As presented earlier, the Shapley allocation guarantees the fairness conditions (i.e., EFF, SYM, DUM, ADD). However, it does not always satisfy the rationality (RAT) condition (which is necessary for stability). According to Lemma I, we have that $\mathrm{ISN}_{\Lambda}$ games are balanced. Moreover, according to [Shapley, 1971, Theorem 7], in balanced games, the Shapley allocation is a member of the core and hence satisfies the rationality condition. Accordingly, for any $\mathrm{ISN}_{\Lambda}$ game, the Shapley allocation guarantees both the stability and fairness. 


\section{ISN GAMES}

In this section we focus on $\mathrm{ISN}_{\Delta}$ games as the class of ISN games with three or more participants and discuss the applicability of the two above mentioned allocation mechanisms for implementing such industrial games.

Example 5 (Neither Core Nor Shapley). Recall the $i j k \mathrm{ISN}_{\Delta}$ scenario from Section 3.2. To have a stable allocation $\left(x_{i}, x_{j}, x_{k}\right)$ in the core, the EFF condition implies $x_{i}+x_{j}+x_{k}=6$ while the RAT condition implies $x_{i}+x_{j} \geq 4 \wedge x_{i}+x_{k} \geq 5 \wedge x_{j}+x_{k} \geq 4$. It is easily checked that these conditions cannot be satisfied simultaneously, hence we can conclude that the core is empty and there exists no way to implement this ISN in a stable manner. Moreover, although the Shapley allocation provides a fair allocation $(13 / 6,10 / 6,13 / 6)$, it is not rational for firms to implement the ISN. E.g., $i$ and $k$ obtain 30/6 in case they defect while according to the Shapley allocation, they ought to sacrifice as they collectively have $26 / 6$.

As illustrated in this example, the Core of $\mathrm{ISN}_{\Delta}$ games may be empty which implies the inapplicability of this solution concept as a general method for implementing ISNs. We generalize the exemplified idea in the following corollary about the implementability of ISN $_{\Delta}$ games in general.

Corollary I (Unimplementability of $\mathbf{I S N}_{\Delta}$ Games). Let $(N, v)$ be an arbitrary ISN $_{\Delta}$ game. The symbiotic relation among industrial agents in $N$ is not generally implementable in a stable manner.

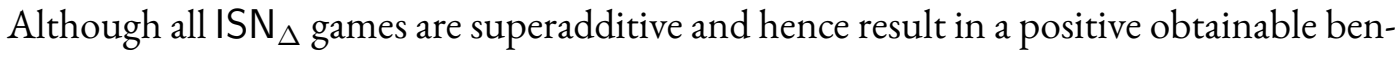
efit, they may be unbalanced (as illustrated in the running example). Accordingly, for any 
unbalanced $\mathrm{ISN}_{\Delta}$ game, the Core is empty. In such cases, the symbiotic relation is not implementable in a stable manner.

Note that the fair implementation of $\mathrm{ISN}_{\Delta}$ games is not always in compliance with the rationality condition. In fact, we lack general methods that guarantee stability and fairness of ISN implementations. So, even if an industrial symbiotic practice could result in collective economic and environmental benefits, it may not last due to instable or unfair implementations. One natural response which is in-line with realistic ISN practices is to employ monetary incentives as a means of coordination.

\subsection{COORdinated ISN}

In realistic ISNs, the symbiotic practice takes place in the presence of economic, social, and environmental policies and under regulations that aim to enforce the policies by nudging the behavior of agents towards desired ones. In other words, while the policies generally indicate whether an ISN is " $\operatorname{good}(\mathrm{bad}$, or neutral)", the regulations are a set of norms that-in case of agents' compliance-result in a spectrum of acceptable (collective) behaviors. Note that the acceptability, i.e., goodness, is evaluated and ought to be verified from the point of view of the policy-makers as community representatives. In this section, we follow this normative approach and aim for using normative coordination to guarantee the implementability of desirable ISNs in a stable and fair manner ${ }^{6}$.

\footnotetext{
${ }^{6}$ In the following, we simply say implementability of ISNs instead of implementability in a fair and stable manner.
} 


\subsection{Normative Coordination of ISNs}

Following [Grossi et al., 20I3; Shoham and Tennenholtz, 1995], we see that during the process of ISN implementation as a game, norms can be employed as game transformations, i.e., as "ways of transforming existing games in order to bring about outcomes that are more desirable from a welfaristic point of view"[Grossi et al., 2013]. To this end, given the economic, environmental, and social dimensions and with respect to potential socioeconomic consequences, industrial symbiotic networks can be partitioned in three classes, namely promoted, permitted, and probibited ISNs. Such a classification can be modeled by a normative socioeconomic policy function $\wp: 2^{N} \mapsto\left\{p^{+}, p^{\circ}, p^{-}\right\}$, where $N$ is the finite set of industrial firms. Moreover, $p^{+}, p^{\circ}$, and $p^{-}$are labels - assigned by a third-party authority-indicating that the ISN among any given agent group is either promoted, permitted, or prohibited, respectively. The three sets $P_{\wp}^{+}, P_{\wp}^{\circ}$, and $P_{\wp}^{-}$consist of all the $\wp$-promoted, -permitted, and -prohibited agent groups, respectively. Formally $P_{\wp}^{+}=\left\{S \subseteq N: \wp(S)=p^{+}\right\}\left(P_{\wp}^{\circ}\right.$ and $P_{\wp}^{-}$can be formulated analogously). Note that $\wp$ is independent of the ISN game among agents in $S$, its economic figures, and corresponding cost values-in general, it is independent of the value function of the game. E.g., a symbiotic relation may be labeled with $p^{-}$by policy $\wp$ —as it is focused on exchanging a hazardous waste-even if it results in a high level of obtainable benefit.

Example 6 (Normative ISNs). In our $i j k$ ISN scenario, imagine a policy $\wp_{1}$ that assigns $p^{-}$ to all the singleton and two-member groups (e.g., because they discharge hazardous wastes in case they operate in one- or two-member groups) and $p^{+}$to the grand coalition (e.g., because in that case they have zero waste discharge). So, according to $\wp_{1}$, the ISN among all the three 
agents is "desirable" while other possible coalitions lead to "undesirable" ISNs.

As illustrated in Example 6, any socioeconomic policy function merely indicates the desirability of a potential ISN among a given group of agents and is silent with respect to methods that enforce the implementability of promoted or unimplementability of prohibited ISNs ${ }^{7}$. The rationale behind introducing socioeconomic policies for ISNs is mainly to make sure that the set of promoted ISNs are implementable in a fair and stable manner while prohibited ones are unstable. To ensure this, in real ISN practices, the regulatory agent (i.e., the regional or national government) introduces regulations - to support the policy - in the form of monetary incentives ${ }^{8}$. This is to ascribe subsidies to promoted and taxes to prohibited collaborations (see [Kakhbod, 2013] for an implementation theory approach on mechanisms that employ monetary incentives to achieve desirable resource allocations). We follow this practice and employ a set of rules to ensure/avoid the implementability of desired/undesired ISNs among industrial agents in $N$ via the allocation of incentives. Such a set of incentive rules can be represented by an MC-Net $\Re=\left\{\partial_{i}:\left(\mathcal{P}_{i}, \mathcal{N}_{i}\right) \mapsto \iota_{i}\right\}_{i \in K}$ in which $K$ is the set of rule indices. Let $\Im(S)$ denote the set of rule indices that are applicable to $S \subseteq N$ (see the notion of applicability in Definition 4$)$. Then, the incentive value for $S$, denoted by $\iota(S)$, is defined as $\sum_{i \in \Im(S)} \iota_{i}{ }^{9}$. The following proposition shows that for any ISN game there exists a set of incentive rules to guarantee the implementability of the ISN in question.

Proposition 4 (Implementability Ensuring Rules). Let $G$ be an arbitrary ISN game among industrial agents in $N$. There exists a set of incentive rules to guarantee the implementability

${ }^{7}$ Note that $\mathrm{ISN}_{\Lambda}$ games are always implementable. So, ISNs' implementability refers to the general class of ISN games including $\mathrm{ISN}_{\Delta}$ games.

${ }^{8}$ See [Meir et al., 20II; Zick et al., 2013] for similar approaches on incentivizing cooperative agent systems. $N$.

${ }^{9}$ This is, a set of incentive rules can be represented also as a cooperative game $\Re=(N, \iota)$ among agents in 
of $G$.

Proof. Recall that according to Proposition 3, $G$ can be represented as an MC-Net. To prove the claim, we provide Algorithm 3 that takes the MC-Net representation of $G$ as a starting point, including a given set of rules, and generates a set of additional rules that guarantee the implementability of $G$.

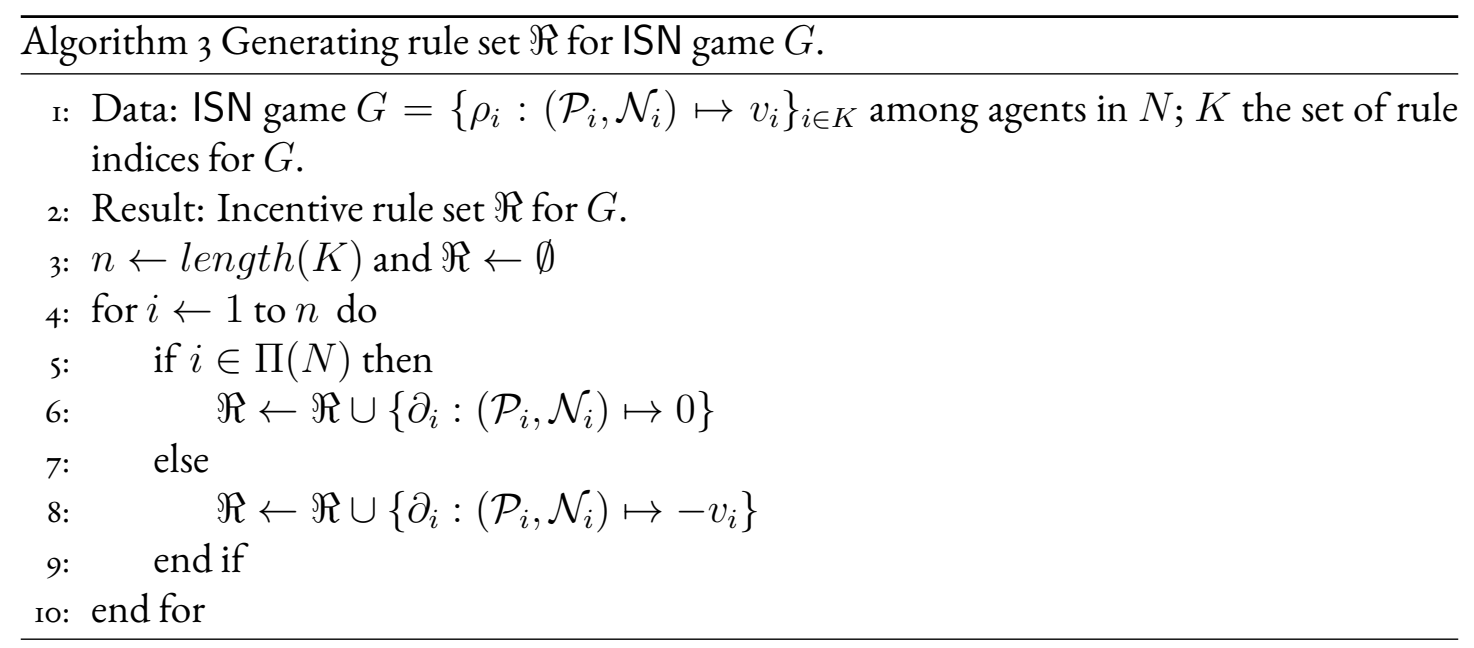

By allocating $-v_{i}$ to rules that are not applicable to $N$, any coalition other than the grand coalition will be faced with a tax value. As the original game is superadditive, the agents will have a rational incentive to cooperate in $N$ and the ISN is implementable in a stable manner thanks to the provided incentive rules.

Till now, we introduced socioeconomic policies and regulations as required (but not yet integrated) elements for modeling coordinated ISNs. In the following section, we combine the idea behind incentive regulations and normative socioeconomic policies to introduce the concept of Coordinated ISN $s(\mathcal{C}-$ ISNs) as a multiagent system for implementing industrial symbiosis. 


\subsubsection{COORDINATED ISNs}

As discussed above, ISN games can be combined with a set of regulatory rules that allocate incentives to agent groups (in the form of subsidies and taxes). We call this class of games, ISNs in presence of coordination mechanisms, or Coordinated ISN $s(\mathcal{C}-I S N s)$ in brief.

Definition 6 (Coordinated ISN Games $(\mathcal{C}-\mathbf{I S N}))$. Let $G$ be an ISN and $\Re$ be a set of regulatory incentive rules, both modeled as MC-Nets among industrial agents in N. Moreover,

for each agent group $S \subseteq N$, let $v(S)$ and $\iota(S)$ denote the value of $S$ in $G$ and the incentive value of $S$ in $\Re$, respectively. A cooperative game $(N, c)$ with $c(S)=v(S)+\iota(S)$ for each agent group $S$ is called a Coordinated ISN game (C-ISN).

Note that as both the ISN game $G$ and the set of regulatory incentive rules $\Re$ are MCNets among industrial agents in $N$, then for each agent group $S \subseteq N$ we have that $c(S)$ is equal to the summation of all the applicable rules to $S$ in both $G$ and $\Re$. Formally, $c(S)=$ $\sum_{i \in \Pi(S)} v_{i}+\sum_{j \in \Im(S)} \iota_{j}$ where $\Pi(S)$ and $\Im(S)$ denote the set of applicable rules to $S$ in $G$ and $\Re$, respectively, and $v_{i}$ and $\iota_{j}$ the value of applicable rules $i$ and $j$ in $\Pi(S)$ and $\Im(S)$, respectively. We sometime use $G+\Re$ to denote the game $C$ as the result of incentivizing $G$ with $\Re$. The next proposition shows the role of regulatory rules in the enforcement of socioeconomic policies.

Proposition 5 (Policy Enforcing Rules). For any promoted ISN game G under policy $\wp$, there exist an implementable $\mathcal{C}-I S N$ game $C$.

Proof. To prove the proposition, for any arbitrary promoted $G$, we require a set of regulatory incentive rules $\Re$ such that its combination with $G$ results in a stable $C$ implementation. The procedure for generating such an $\Re$ is presented in Algorithm 3. 
Analogously, similar properties hold for avoiding prohibited ISNs or allowing permitted ones. Avoiding prohibited ISNs can be achieved by making the $\mathcal{C}-$ ISN (that results from introducing regulatory incentives) unimplementable. On the other hand, allowing permitted ISNs would be simply the result of adding an empty set of regulatory rules. The presented approach for incentivizing ISNs, is advisable when the policy-maker is aiming to ensure the implementability of a promoted ISN in an ad-hoc way. In other words, an $\Re$ that ensures the implementability of a promoted ISN $G_{1}$ may ruin the implementability of another promoted ISN $G_{2}$. This highlights the importance of some structural properties for socioeconomic policies that aim to foster the implementability of desired ISNs. As we discussed in Section 3.2, we aim for implementing ISNs such that the rationality axiom will be respected. In the following, we focus on the subtleties of socioeconomic policies that are enforced by regulatory rules. The question is, what are the requirements of a policy that can ensure the rationality of staying in desired ISNs? We first show that to respect the rationality axiom, promoted agent groups should be disjoint. We illustrate that in case the policy-maker takes this condition into account, industrial agents have no economic incentive to defect an implementable promoted ISN.

Proposition 6 (Mutual Exclusivity of Promoted ISNs). Let $G_{1}$ and $G_{2}$ be arbitrary ISNs, respectively among promoted (nonempty) agent groups $S_{1}$ and $S_{2}$ under policy $\wp$ (i.e., $S_{1}, S_{2} \in$ $P_{\wp}^{+}$). Moreover, let $\Re_{1}$ and $\Re_{2}$ be rule sets that ensure the implementability of $G_{1}$ and $G_{2}$, respectively. For $i \in\{1,2\}$, defecting from $\mathcal{C}-I S N C_{i}=G_{i}+\Re_{i}$ is not economically rational for any agent $a \in S_{i}$ iff $S_{1} \cap S_{2}=\emptyset$.

Proof. “ $\Rightarrow$ ”: Suppose $S_{1} \cap S_{2} \neq \emptyset$. Accordingly, we have an agent $a$ which is both a member of $S_{1}$ and $S_{2}$. For $a$ it is rational to defect either $S_{1}$ or $S_{2}$ as both are among the promoted 
agent groups under $\wp$ (see that the procedure for ensuring implementability goes through the introduction of penalizing rules).

“ $\Leftarrow$ : Suppose $S_{1}$ and $S_{2}$ are disjoint promoted agent groups under $\wp$. As $\Re_{1}$ and $\Re_{2}$ can respectively ensure the implementability of these two groups and based on Proposition 3, we have that ISNs among firms in $S_{1}$ and $S_{2}$ are both implementable in a stable manner. Hence, they satisfy the rationality axiom. Moreover, as the two agent groups share no agent, there will be no economic incentive to deviate between the two stable ISNs.

Accordingly, given a set of industrial agents in $N$ and a socioeconomic policy $\wp$ we directly have that:

Proposition 7 (Minimality of Promoted ISNs). For $n=\left|P_{\wp}^{+}\right|$if $\bigcap_{i=1}^{n} S_{i} \in P_{\wp}^{+}=\emptyset$ then any arbitrary $S_{i} \in P_{\wp}^{+}$is minimal (i.e., $S_{i}^{\prime} \notin P_{\wp}^{+}$for any $S_{i}^{\prime} \subset S_{i}$ ).

Roughly speaking, the exclusivity condition for promoted agent groups entails that any agent is in at most one promoted group. Hence, deviation of agents does not lead to a larger promoted group as no promoted group is part of a promoted super-group, or contains a promoted sub-group. In the following, we show that the mutual exclusivity condition is sufficient for ensuring the implementability of all the ISNs that take place among promoted groups of firms.

Theorem 4 (Conditioned Implementability). Let $G$ be an arbitrary $\mathrm{ISN}_{\Delta}$ game underpolicy $\wp$ among industrial agents in $N$. If $S \cap S^{\prime}=\emptyset$ for any arbitrary $S, S^{\prime} \in P_{\wp}^{+}$, then there exists a set of regulatory rules $\Re$, such that all the promoted symbiotic networks are implementable in the coordinated ISN defined by $C=G+\Re$. Moreover, any ISN among probibited agent groups in $P_{\wp}^{-}$will be unimplementable. 
Proof. To prove Theorem 4, we provide a method to generate such an implementability ensuring set of rules. We start with an empty $\Re$. Then for all $n$ promoted $S_{i} \in P_{\wp}^{+}$, we call Algorithm 3. Each single run of this algorithm results in an $\Re_{i}$ that guarantees the implementability of the industrial symbiosis among the set of firms in the promoted group $S_{i}$. As the set of promoted agent groups comply to the mutual exclusivity condition, the unification

of all the regulatory rules results in a general $\Re$. Formally, $\Re=\bigcup_{i=1}^{n} \Re_{i}$. Moreover, as the algorithm applies taxation on non-promoted groups, no ISN among prohibited agent groups will be implementable.

Example 7 (ijk as a Normatively Coordinated $\mathcal{C}-\mathbf{I S N})$. Recalling the ISN scenario in Example 6, the only promoted group is the grand coalition while other possible agent groups are prohibited. To ensure the implementability of the unique promoted group and to avoid the implementability of other groups, the result of executing our algorithm is $\Re=\left\{\partial_{1}\right.$ : $\left.(i j, k) \mapsto-4, \partial_{2}:(i k, j) \mapsto-5, \partial_{3}:(j k, i) \mapsto-4\right\}$. In the $\mathcal{C}-$ ISN that results from adding $\Re$ to the original ISN, industrial symbiosis among firms in the promoted group is implementable while all the prohibited groups cannot implement a stable symbiosis.

\subsubsection{Realized ISNs and Budget-Balancedness}

As we mentioned in the beginning of Section 3.5, regulations are norms that in case of agents' compliance bring about the desired behavior. For instance, in Example 7, although according to the provided tax-based rules, defecting the grand coalition is not economically rational, it is probable that agents act irrationally-e.g., due to trust-/reputation-related issues-and leave the promoted group. This results in possible normative behavior of a $\mathcal{C}-I S N$ with respect to an established policy $\wp$. So, assuming that based on evidences the set of implemented ISNs are 
realizable, we have the following abstract definition of $\mathcal{C}-$ ISN's normative behavior under a socioeconomic policy.

Definition 7 (C-ISN's Normative Behavior). Let $C$ be a $\mathcal{C}-I S N$ among industrial agents in $N$ under policy $\wp$ and let $E$ be the set that includes all the implemented ISNs among agents in N.E is called the evidence set. We say the behavior of $C$ complies to $\wp$ according to $E$ iff $E=P_{\wp}^{+}$; and violates it otherwise.

Given an ISN under a policy, we introduced a set of regulatory rules to ensure that all the promoted ISNs will be implementable. However, although providing incentives makes them implementable, the autonomy of industrial agents may result in situations that not all the promoted agent groups implement their ISN. So, although we can ensure the implementability of all the promoted ISNs, the real behavior may deviate from a desired one. As our introduced method for guaranteeing the implementability of ISNs among promoted agent groups is mainly tax-based, if a $\mathcal{C}$-ISN violates the policy, we end up with collectible tax values. In such cases, our tax-based method can become a balanced-budget monetary incentive mechanism (as discussed in [Guo and Conitzer, 2008; Li et al., 2003; Phelps et al., 2010]) by employing a form of "Robin-Hood" principle and redistributing the collected amount among promoted agent groups that implemented their ISN. In the following, we provide an algorithm that guarantees budget-balancedness by means of a Shapley-based redistribution of the collectible tax value among agents that implemented promoted ISNs.

The correctness of Algorithm 4 is established in Proposition 8.

Proposition 8 (Budget Balancedness and Fairness). Let $C=G+\Re$ be a $\mathcal{C}-$ ISN among industrial agents in $N$ under policy $\wp$ such that all the ISNs among promoted groups are implementable (using the provided method in Theorem 4) and let E be the set of implemented 


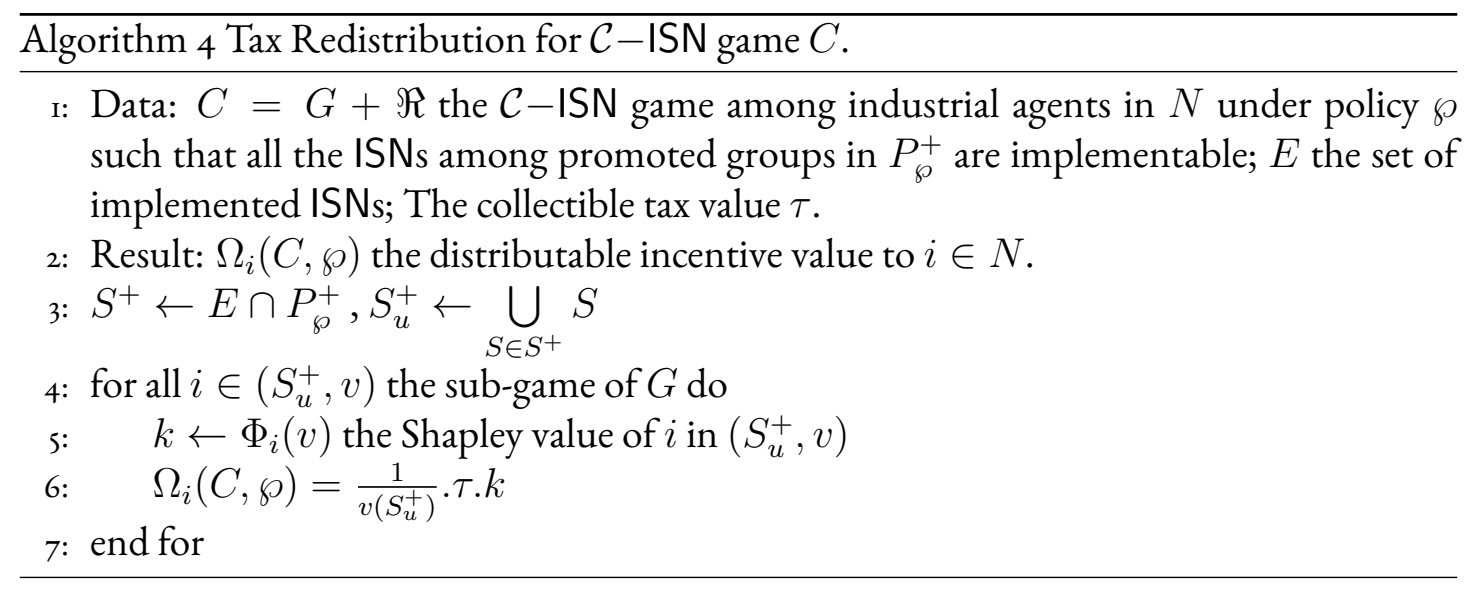

ISNs. For any $\mathcal{C}-\mathrm{ISN}$, the incentive values returned by Algorithm 4 ensures budget balancedness while preserving fairness (i.e., EFF, SYM, DUM, and ADD).

Proof. To have budget balancedness, we have to show that the total collectible $\mathrm{e}^{\mathrm{ro}}$ tax value (using the provided method in Theorem 4 ) is equal to allocated subsidies. If the $\mathcal{C}-$ ISN is $\wp$-compliant, this is obvious as $\tau$ is equal to zero (thanks to the implementation of all the promoted ISNs). When the $\mathcal{C}-$ ISN is $\wp$-violating, we use the Shapley value of each agent that contributes to the sub-game of implemented promoted ISNs. As we employ a Shapleybased method, the monetary incentive is budget-balanced thanks to the EFF property and in addition preserves the other three properties (i.e., SYM, DUM, and ADD).

Note that the redistribution phase takes place after the implementation of the ISNs and with respect to the evidence set $E$. Otherwise, there will be cases in which the redistribution process provides incentives for agent groups to defect the set of promoted collaborations. Moreover, we highlight that the use of an MC-net representation of games enables calculating the Shapley value in a scalable manner-in Algorithm 4 (see [Ieong and Shoham, 2005] for

\footnotetext{
${ }^{\text {Io }}$ Considering a disposal account - under control of the regulatory agent-for each firm, it is reasonable to assume that collectible $\tau$ is equal to collected $\tau$.
} 
complexity results). In particular, the Shapley value of an agent in MC-nets is equal to the summation of its Shapley values in each MC-net rule. Accordingly, as in each rule the value can be computed in linear time (in the pattern of the rule), the Shapley value computation for the whole game will be linear in the size of the input.

\subsection{Concluding Remarks}

This chapter provides a coordinated multiagent system — rooted in cooperative game theoryfor implementing ISNs that take place under a socioeconomic policy. The use of rule-based MC-Net representation of cooperative games enables combining the game with a set of policies and regulations in a natural way. This work also provides algorithms that generate regulatory rules to ensure the implementability of "good" symbiotic collaborations in the eye of the policy-maker. This extends previous work that merely focused on operational aspects of industrial symbiotic relations-as we introduce the analytical study of the regulatory aspect of ISNs. Finally, it introduces a method for redistribution of collectible tax values. The presented method ensures the budget-balancedness of the monetary incentive mechanism for coordination of ISNs in the implementation phase.

In practice, such a framework supports decision-makers in the ISN implementation phase by providing operational semantics as a toolset for reasoning about the implementability of a given ISN in a fair and stable manner. Moreover, it supports policy-makers aiming to foster socioeconomically desirable ISNs—by providing algorithms that generate the required regulatory rules. Finally, it shows that MC-Net is an expressive representation framework for applying normative coordination mechanisms to multiagent systems.

This chapter focuses on a unique socioeconomic policy and a set of rules to ensure it. 
In this regard, one question that deserves investigation is the possibility of having multiple policies and thus analytical tools for policy option analysis [Mehryar et al., 2017] in ISNs. Such a framework assists the ranking and investigation of the applicability of a set of policies in a particular ISN scenario. Along this line, we aim to generate a regulation toolbox for ISN policy-makers-since a single regulation may be incapable of ensuring all the desired collaborations under potentially conflicting policies. In that case, possible conflicts among regulations can be resolved using prioritized rule sets (inspired by methods for dealing with potential extensions in argumentation theory [Kaci and van der Torre, 2008; Modgil and Prakken, 2013]). Accordingly, we will have distinguishable potential ISN worlds such that in each a maximal set of promoted ISNs is implementable.

In future work, we also aim to focus on administration of ISNs. Then, informationsharing issues [Carter et al., 2002; Fraccascia and Yazan, 2018] and compliance of involved agents to their commitments will be main concerns for automated trading and business implementations in multiagent industrial symbiosis systems [Ash, 2004]. For that, we plan to model ISNs as normative multiagent organizations [Boissier and Van Riemsdijk, 20I3; Yazdanpanah et al., 2016] and integrate data-driven coordination techniques [Wardi et al., 2018]. Thence, we can rely on norm-aware organization frameworks that focus on operation of normative organizations [Aldewereld et al., 2007; Dastani et al., 2017a,b] to monitor the organization's behavior. Finally, we aim to illustrate the validity of our formally verified framework using realistic case studies and multiagent-based simulations [Fraccascia et al., 2017a]. 



\section{With great power comes great responsibility.}

(the exact origin is unknown)

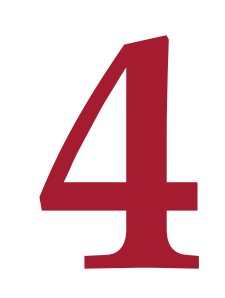

Allocating Transaction Costs in Industrial Symbiosis

This Chapter discusses the role of Transaction Cost (TC) ECONOMics in Industrial Symbiosis Institutions (ISI) and provides a fair and stable mechanism for TC allo- 
cation among the involved firms in a given ISI. In principle, industrial symbiosis-as an implementation of the circular economy paradigm in the context of industrial relation-is a practice aiming at reducing the material/energy footprint of the firm. The well-engineered form of this practice should also decrease the transaction costs at a collective level. This can be achieved using information systems for: identifying potential synergies, evaluating mutually beneficial ones, implementing the contracts, and governing the behavior of the established relations. Then the question is "how to distribute the costs for maintaining such an information system in a fair and stable manner?" We see such a cost as a collective transaction cost and employ an integrated method rooted in cooperative game theory and multiagent systems research to develop a fair and stable allocation mechanism for it ${ }^{\mathrm{I}}$. The novelty is twofold: in developing analytical methods for capturing the dynamics of transaction costs in industrial symbiosis and in presenting a novel game-theoretic mechanism for its allocation in multiagent industrial symbiosis institutions. While the former contributes to the theories of industrial symbiosis (methodological contribution), the latter supports decision makers aiming to specify fair and stable industrial symbiosis contracts (practical contribution). In a broader perspective, such an approach contributes to the intersection of multiagent technologies, industrial engineering, and institutional economics.

\section{I Introduction AND Motivation}

Industrial symbiosis is a transitional business model to shift from linear economy paradigms towards implementing the concept of circular economy-in the context of industrial rela-

IThe content of this chapter is mainly based on "Vahid Yazdanpanah, Devrim Murat Yazan, and W. Henk M. Zijm. Dynamics and allocation of transaction cost in multiagent industrial symbiosis. Computers E\& Industrial Engineering, page (submitted), 2019d" [Yazdanpanah et al., 2019d]. 
tions/networks. In principle, the aim is to facilitate the circulation of reusable resources among the network members [Chertow, 2007; Yazan et al., 2016]. Realizing such a form of collaboration requires methods for identifying potential matches [van Capelleveen et al., 20I8], evaluating them to generate mutually beneficial instances [Yazdanpanah et al., 2019e], implementing the cost-sharing schemes in bilateral contracts [Yazdanpanah and Yazan, 2017], and decentralized governance of the established relations [Yazdanpanah et al., 20i6]. As elaborated in [Yazdanpanah et al., 20I8] problematic situations occur when we move from bilateral relations to multilateral forms in multiagent industrial symbiosis. Dealing with such problems requires: (I) developing practical methods able to capture collective-level concepts, such as collectively realized transaction costs, (2) practice-oriented semantics to reason about the link between individual (firm-level) concerns and the collective (institution-level) attributes, and (3) implementable mechanisms to guarantee desirable collective attributes with no harm to firm-level concerns. This work aims to address this gap by focusing on the nature of collective transaction costs in industrial symbiosis and developing a contextualized allocation mechanism that guarantees game-theoretical fairness and stability properties.

\section{I.I Conceptual Analysis: On the Nature and Dynamics of TC in IS}

As originally introduced by [Williamson, 1979], transaction costs play a key role in the establishment and stability of different forms of contractual relations. This is also the case for industrial symbiosis relations, both in bilateral and multilateral forms. For instance, when an industrial cluster manager aims to share the collective costs for maintaining the shared environments among the cluster members, she should take into account the firm-level concerns. One general approach is to see the contribution of each firm to the collective as a measure 
for making such cost/benefit allocation decisions [Billera and Heath, 1982]. In the case of IS, while we have such contribution-aware tools for allocating costs in bilateral IS relations [Yazdanpanah and Yazan, 20I7], they fail to guarantee some basic properties such as collective rationality (related to whether firms have any incentive to leave the collaboration) on a multilateral network level [Yazdanpanah et al., 20I8]. In practice, such a disadvantage results in inefficient deployment of an IS management platform in real-life industrial symbiosis networks.

To develop an efficient technique for dealing with this problem, it is crucial to understand the context and see what are transaction costs in IS. In multiagent industrial symbiosis, the main elements that contribute to the transaction cost are costs involved in market/partner searching, negotiation costs, and the relation enforcement cost [Chertow and Ehrenfeld, 20I2; Fraccascia and Yazan, 2018]. We argue that in modern industrial symbiosis, and thanks to (online) IS information systems/platforms, the total IS transaction cost boils down to costs for establishment and maintenance of the information system that is responsible for handling the searching/matching process, for supporting/automating the negotiations, and for synthesizing the required enforcement measures. Then the question is about finding methods to distribute this total cost among the involved firms, such that it would be fair with respect to each firm's contribution. That means that, after a basic payment for getting involved in a platform, firms that may gain more in a given network, are expected to pay more for upcoming transaction costs. In other words: "with great power comes great responsibility". This perspective supports the so called fairness notions- proposed in cooperative game theorythat agents' individual benefit/cost share oughts to reflect their contribution to the collective benefit/cost [Deng and Papadimitriou, 1994; Young, 1985a]. 
While the idea to take into account each agent's contribution provides a basis for allocating the costs in multiagent industrial symbiosis, we lack methods for defining the value of each and every coalition of firms, involved in the network ${ }^{2}$. Such an input is crucial for applying standard fair allocation mechanisms (e.g., the notion of the Shapley value [Roth and Verrecchia, 1979; Shapley, 1953]). In response, developing a method that characterizes IS as a game is the first objective of this chapter. The result of this first step will be a game-form that in turn enables the application of game theoretic solution concepts for allocating the transaction cost. Technical details will follow in Section 4.2. To formulate a realistic gamebased on which the transaction cost can be allocated-one question that is crucial to address is "what is the nature of transaction cost in IS practices?". In the IS literature [Fraccascia and Yazan, 2018; Yazdanpanah et al., 2019e], costs for operationalizing IS are categorized as costs for transporting the resource, for treating them by means of recycling/preparation processes, and finally transaction costs as discussed above. In principle, transportation and treatment costs are based on the realization of physical facts and interactions ${ }^{3}$. For instance, the physical distance between the firms is one of the key measures that determines the transportation cost and hence can be a fair basis for allocating the collective transportation cost among the involved firms. For treatment costs—as the total cost that results from recycling, drying, and categorizing-we also have physical processes that consume resources (e.g., electricity and

\footnotetext{
${ }^{2}$ In the game-theoretic language, the characteristic function of the cooperative multiagent industrial symbiosis is not well-defined with respect to the context of industrial symbiosis and its constraints.

${ }^{3}$ To reason about the essence of transaction costs, we use the terminology of [Searle, 2005] and form our perspective based on the categorization of physical and institutional facts and their corresponding acts. In brief, physical facts are about the valuation of a variable in the observable world (e.g., the quantity of a resource or the distance between two firms) while institutional facts are about the invisible world of concepts, definable in a given context (e.g., the fact that a resource is needed or that a firm is powerful). In turn, physical acts may change the value of physical facts while institutional acts affect the institutional facts. We abstract from the reasoning structures that relate the two disjoint sets of facts/acts. See [Yazdanpanah et al., 2016] for further elaborations on such a conceptualization in the context of industrial symbiosis.
} 
diesel cost of a recycling plant). However, the transaction cost is not based on physical facts, but mainly is the aggregation of costs of institutional acts, e.g., negotiation costs and corresponding costs for establishing monitoring/enforcement mechanisms. These acts are on the one hand required to enable or keep track of physical processes (hence have a physical connotation) and on the other hand are related to the structure of interfirm connections (hence have an institutional nature). Therefore, it would be reasonable to consider a form of dual-nature physical-institutional IS value as a basis for allocating the collective transaction cost among the involved firms. This way of capturing the institutional dimension of the transaction cost is in-line with the original studies on this notion, as presented in [Silverman, 1999; Williamson, 1979, 1993]. Although the line of reasoning is straightforward, capturing such a perspective in a practical tool for allocating the collective transaction costs in multiagent industrial symbiosis is an open problem ${ }^{4}$. Next, we elaborate on our approach on tailoring formal multiagent organizational methodologies [Horling and Lesser, 2004] for modeling industrial symbiosis as an institution and for developing an operationally feasible transaction cost allocation mechanism.

\section{I.2 Institutional Approach: On How to Address the Problem}

We see multiagent industrial symbiosis as a practical manifestation of (well-designed) industrial institutions and aim to model it using game-theoretic methods, able to capture both the physical and the institutional contributions of involved firms s. Such a formal approach en-

\footnotetext{
${ }^{4}$ As highlighted by [Yazdanpanah et al., 20I8], some standard allocation mechanisms are inapplicable for IS implementation due to operational complexities-embedded in real-life multiagent industrial relations.

${ }^{5}$ We use the term institution as a general reference to a collective of entities behaving in a systematic manner, under an emerged or established coordination mechanism. Then a well-designed institution is one in which the mechanism is engineered such that some (collectively desirable) properties hold [Rubino et al., 2005]. This would be distinguishable from the stronger notion of organization where we have an explicit representation
} 
ables employing institutional economics methods for guaranteeing desirable properties at the collective level, e.g., see [Yazdanpanah et al., 20I8] for how a regulatory agent can influence the feasibility of multiagent industrial symbiosis by means of incentive engineering techniques.

In relation to the focus of this chapter, i.e., the collective transaction cost and its allocation among the involved firms, the fairness property is concerned with capturing the contributions of firms as a basis for cost allocation. While physical acts/facts (e.g., distance and quantifiable used energy) determine a base for computing fair allocation of (physical) transportation/treatment costs, a fair allocation of the collective transaction cost calls for a notion to capture the institutional contribution of agents as well. To that end, we provide a formal account of the transaction cost economics in industrial symbiosis (based on computational organization theory and industrial institutions). This results in the introduction of the notion of industrial symbiosis index as a measure for capturing the physical and institutional contribution of firms. In turn, this notion will be a base for developing a fair and stable transaction cost allocation mechanism - rooted in the literature on fair division mechanisms [Pratt and Zeckhauser, 1990; Thomson, 1983]. Finally, we elaborate on potential questions to be solved using the provided methodological foundation.

\subsection{Modeling Industrial Symbiosis Institutions}

To model IS institutions, we build upon a graphical representation of cooperative gamesalso known as graphical games [Kearns, 2008] or graph-restricted games [Myerson, 1977]. Such a representation is a natural choice as it reflects the established relations among the firms and allows the application of standard fair division methods for sharing the collective trans-

of roles, organizational goals/preferences, organizational structures, and interaction protocols [Baldoni et al., 20Io; Van den Broek et al., 2005]. 
action cost, among the members of the institution ${ }^{6}$. As a first step, we use graph-theoretic notions to determine a realistic characteristic function for the game-theoretic representation of industrial symbiosis. Then, adding an allocation mechanism results in our formal notion of industrial symbiosis institution.

\subsection{Preliminary Notions and Definitions}

We recall basicgame theoretic notions and the definition of graphical games based on [Chalkiadakis et al., 20II; Mas-Colell et al., I995; Myerson, 1977].

Cooperative Games: A (transferable utility) cooperative game on a finite set of agents $\Gamma$ is a tuple $\langle\Gamma, f\rangle$ where the game's characteristic function $f: 2^{\Gamma} \mapsto \mathbb{R}$ is such that $f(\emptyset)=0$.

Graphical Games: A graphical (transferable utility) cooperative game on a finite set of agents/vertices $\Gamma$ is a triple $\langle\Gamma, W, f\rangle$ where $W$ is a $|\Gamma| \times|\Gamma|$ real-valued weight matrix (representing the weights of edges between vertices in $\Gamma$ ) and the game's $W$-restricted characteristic function $f^{W}: 2^{\Gamma} \mapsto \mathbb{R}$ is such that $f(\emptyset)=0$. We say $f$ is restricted to $W$ as it determines the value of any coalition $S \subseteq \Gamma \backslash \emptyset$ with respect to $W$. Such a general formalization allows further tailoring in the context of industrial symbiosis.

Allocation Mechanisms: For a given cooperative game $\mathcal{G}=\langle\Gamma, f\rangle$, a (single-point) allocation mechanism $\mathcal{M}$ maps a real-valued tuple $\mathcal{M}(\mathcal{G}) \in \mathbb{R}^{|\Gamma|}$ to the pointed game. The $i$-th element of the allocation tuple $\mathcal{M}(\mathcal{G})=\left\langle a_{1}, a_{2}, \ldots, a_{|\Gamma|}\right\rangle$ is the share of agent $i \in \Gamma$ according to $\mathcal{M}$ and with respect to $\mathcal{G}$. The term share can be interpreted-with respect to the context-as the amount to be paid or gained by $i$. We later discuss various properties that

\footnotetext{
${ }^{6}$ Through the course of this work, we may refer to firms as agents-following the convention in computational economics. This would be to see any industrial symbiosis institution as an environment that supports the collaborative interaction of a set of autonomous, rational decision-makers in charge of the involved firms.
} 
such a mechanism can hold or bring about.

\subsubsection{From Weighted Connectivity Graphs to Cooperative Graph Games}

To determine how the transaction cost can be allocated among the firms based on their physical and institutional contributions, we take the graph that represents the established ${ }^{7}$ symbiotic relations and obtained cost reductions as an input. Given such a graph, we formulate a game-theoretical representation that in turn results in inducing the physical as well as the institutional contribution of individual firms.

Definition 8 (IS Graph). An IS graph is a tuple $\langle\Gamma, W\rangle$, where $\Gamma$ is the set of vertices, representing $|\Gamma|$ firms and $W$ is the symmetric $|\Gamma| \times|\Gamma|$ matrix of positive real valued weights, representing the cost reduction values. There exists a weighted undirected edge between distinct firms $i, j \in \Gamma$, representing their established symbiotic relation, only if $W_{i, j} \neq 0$. Moreover, for any $i \in \Gamma$ we have that $\sum_{j \in \Gamma} W_{i, j}>0$ (connected) and that $W_{i, i}=0$ (loop-free).

To have a concise and contextualized representation, we do not require an explicit set of edges as it could be derived based on $W$. The same holds for requiring the graph to be loopfree and connected. Basically, loops and disconnected firms can be excluded as in such cases the transaction cost (hence its allocation) is meaningless. This results in a realistic representation in which unfeasible relations/edges (which otherwise could be represented by negative or zero weights) are excluded.

${ }^{7}$ Note that the reasoning about such a cost allocation takes place in a retrospective manner and after the establishment of industrial symbiosis relations. It is important to note that, other than in the former chapters, we now speak of an in principle realized collaboration. Thus, it is reasonable to assume that the graph $W$ can be derived from such a realized IS. 
In the context of IS, $W_{i, j}\left(=W_{j, i}\right)$ reflects the realized net benefit-in terms of collectively obtained cost reductions-of the symbiotic relation between firms $i$ and $j$ on a given (quantity of) resource $r$. As discussed in [Yazdanpanah et al., 2019e], such a collective benefit can be computed by deducing the total operational cost of the relation (for treatment and transportation of $r$ ) from the total traditional costs (for discharging $r$ on the provider side of the relation and purchasing traditionally-used inputs-substituted by $r$ in the realized relation-on the receiver side). In principle, matrix $W$ would be the basis for formulating both the physical IS game (reflecting obtainable benefits) and the institutional game (modeling the institutional power of firms in the cluster).

Definition 9 (Physical IS Game). A graphical physical IS game is a triple $\langle\Gamma, W, v\rangle$, where $G=\langle\Gamma, W\rangle$ is an IS graph and for any group of firms $S \subseteq \Gamma$ with $|S|>1$, the characteristic function $v(S)$ is equal to $\frac{1}{2} \sum_{i, j \in S} W_{i, j}$. By convention, for any $S$ with $|S| \leq 1$, $v(S)=0$. Then in the normalized characteristic function, denoted by $\bar{v}$, we have that $\bar{v}(S)=v(S) / v(\Gamma)$.

Intuitively, in a physical IS game, the value of a group $S$ is equal to the summation of the weights of the edges that connect the group members. Note that in the symmetric matrix $W$, the weight of the edge between arbitrary group members $i$ and $j$ appears both at $W_{i, j}$ and $W_{j, i}$ (both representing the obtained cost reduction as a result of the IS relation between firms $i$ and $j$ ). Hence, $v(S)$, the value of a group $S$, is equal to $\frac{1}{2} \sum_{i, j \in S} W_{i, j}$.

Example 8. To demonstrate the applicability of our approach, we use a case study (adopted from a realistic industrial cluster $^{8}$ ). See Figure 4.I for an illustration of the IS graph. In this

\footnotetext{
${ }^{8}$ The adopted case is one of the successful implementations of industrial symbiosis networks in the SHAREBOX project [SHAREBOX, 2019]. Due to confidentiality concerns, we omitted the company names and modified some values.
} 
graph, the value on each edge reflects the benefit (in terms of cost reductions) that resulted from the symbiotic relation, realized between the nodes that it connects. While such values represent the physical dimension of an IS practice, the structure of the graph is what we later use to formulate the institutional importance of each node/firm.

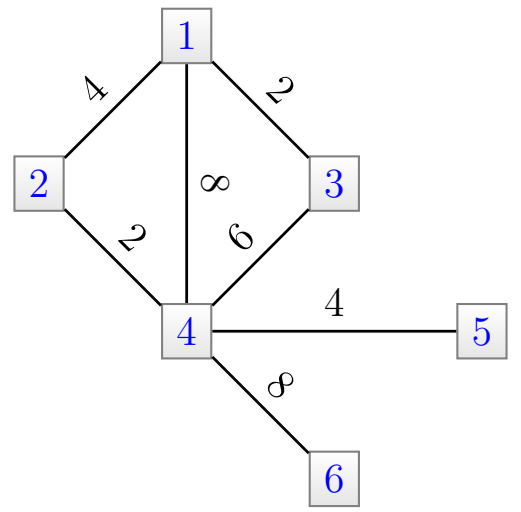

Figure 4.1: Connectivity graph of the involved firms in the IS cluster. Each node represents a firm and the value on each edge represents the amount of cost reduction, obtained as a result of the collaboration between the firms that the edge connects. Values are presented as utils. A util can be interpreted as any form of transferable utility, e.g., a util me be equal to 100 Dollars.

The value of any singleton or empty coalition is 0 while for any $S$ with $|S|>1$, we calculate the value by simply adding up the weights of the edges that connect any two member of $S$. For instance, $v(\{1,2,3,4\})=22$ and $v(\Gamma=\{1, \ldots, 6\})=34$. For the same coalitions, the normalized values are respectively $\frac{22}{34}$ and $\frac{34}{34}$. These normalized values will be later employed for aggregation of the physical game with a game that represents the institutional power of firms in such clusters.

Below, we present game theoretic properties of the physical IS games.

Proposition 9 (Properties). Let $\mathcal{G}=\langle\Gamma, W, v\rangle$ be a physical IS game. Then:

(I) for any coalitions $S \subset T$, we have that $v(S) \leq v(T)$ (monotonicity). 
(2) for any disjoint coalitions $S$ and $T$, we have that $v(S \cup T) \geq v(S)+v(T)$ (superadditivity).

(3) for any coalitions $S$ and $T$, we have that $v(S \cup T)+v(S \cap T) \geq v(S)+v(T)$ (convexity/super-modularity).

Proof. (I) imagine a firm $i$ in $T \backslash S$. If $i$ is connected to a member of $S$ or $T \backslash S$, it contributes to the value of $T$. Otherwise, it has the added value of 0 . In both cases, part (I) is true. (2) if there exist a direct edge connecting a member of $S$ to a member of the disjoint coalition $T$, then $v(S \cup T)$ increases; otherwise, it is equal to $v(S)+v(T)$, thanks to non-negative weights. (3) In case the two sets are disjoint, it follows from part (2). Otherwise, the two coalitions have nonempty intersection with either positive or zero value. Note that, thanks to non-negative weights, potential edges from the intersection to either $S \backslash S \cap T$ or to $T \backslash S \cap T$ are not destructive to the claim. The same holds for the fact that the edges in the intersection are counted twice, both in $v(S \cap T)$ and in $v(S \cup T)$.

These properties result in the following practical result that the collective value of the grand coalition $\Gamma$ can be shared among the firms such that no coalition has an incentive to defect the collaboration.

Theorem 5 (Nonempty Core). Let $\mathcal{G}=\langle\Gamma, W, v\rangle$ be a physical IS game. Then there exists an allocation mechanism $\mathcal{M}$ such that in $\mathcal{M}(\mathcal{G})=\left\langle a_{1}, a_{2}, \ldots, a_{|\Gamma|}\right\rangle$, we have (I) $\sum_{i \in \Gamma} a_{i}=v(\Gamma)$ (Efficiency) and (2) for any $S \subseteq \Gamma, \sum_{i \in S} a_{i} \geq v(S)$ (Coalitional Rationality).

Proof. The two clauses in this theorem axiomatize the notion of core-nonemptiness [Shapley and Shubik, 1969]. Then, the convexity property (in Proposition 9) in combination with 
the well-established Bondareva-Shapley theorem [Bondareva, 1963; Shapley, 1967] ensures the nonemptiness of the core, and accordingly the existence of a mechanism to generate the allocation.

\subsubsection{From Cooperative Games to Industrial Institutions}

In addition to the physical game-based on which we can induce the physical contributions that firms can bring about through a bilateral exchange of resources-the next step is to present a basis for capturing the institutional contribution of firms. For such a purpose, we cannot rely on cost reduction values (obtained thanks to operationalizing the relations) basically because transaction costs are non-operational, but have an institutional nature. Therefore, we employ the notion of closeness centrality adopted from the literature on communication networks [Bavelas, 1950] to capture the institutional power of firms, and as the basis for defining the characteristic function of the IS institutional game. Basically, using the notion of closeness centrality, we can capture the intuition that firms that interact with many other firms should contribute relatively more to the collective transaction costs than firms that interact with only a few (or just one other) partner(s). In a graph on the set of vertices

$\Gamma$, the closeness centrality of a vertex $i \in \Gamma$, denoted by $\mathfrak{C}(i)$, is equal to $\frac{|\Gamma|-1}{\sum_{j \in \Gamma \backslash\{i\}} d(i, j)}$ where the distance function $d: \Gamma \times \Gamma \mapsto \mathbb{N}^{+}$returns the shortest distance between $i$ and $j$. More explicitly, $d(i, j)=d(j, i)$ returns the minimum number of edges passed to reach $j$ from $i$. Recalling the presented IS graph in Figure 4.I, we have that $\mathfrak{C}(1)=\frac{5}{7}, \mathfrak{C}(2)=\mathfrak{C}(3)=\frac{5}{8}$, $\mathfrak{C}(4)=\frac{5}{5}$, and $\mathfrak{C}(5)=\mathfrak{C}(6)=\frac{5}{9}$.

Then based on this notions, we formulate the institutional IS game as follows.

Definition ro (Institutional IS Game). A graphical institutional IS game is a triple $\langle\Gamma, W, \iota\rangle$, 
where $G=\langle\Gamma, W\rangle$ is an IS graph and for any group of firms $S \subseteq \Gamma$ with $|S|>0$, the characteristic function $\iota(S)$ is equal to $\sum_{i \in S} \mathfrak{C}(i)$. By convention, $\iota(\emptyset)=0$. Then in the normalized characteristic function, denoted by $\bar{\iota}$, we have that $\bar{\iota}(S)=\iota(S) / \iota(\Gamma)$.

Due to the additive formulation of $\iota$, we have the following properties for institutional IS games.

Proposition Io (Properties). Let $\mathcal{G}=\langle\Gamma, W, \iota\rangle$ be an institutional IS game. Then $\mathcal{G}$ is (I) monotonic and (2) convex/super-modular (defined analogously to Proposition 9).

Proof. Given that $\mathfrak{C}(i)$ is non-negative for all $i \in \Gamma$ and the formulation of $\iota(S)$ as the summation of $\mathfrak{C}(i)$ for all $i \in S$, monotonicity is trivial. For convexity, it suffices to decompose $\iota(S \cup T)$. We have that $\iota(S \cup T)$ is equal to $\sum_{i \in S \cup T} \mathfrak{C}(i)=\sum_{j \in S} \mathfrak{C}(j)+\sum_{k \in T} \mathfrak{C}(k)-$ $\sum_{l \in S \cap T} \mathfrak{C}(l)$, hence $\iota(S \cup T)+\iota(S \cap T)=\iota(S)+\iota(T)$, which satisfies the convexity condition.

Immediate to this, we have the existence of an efficient and coalitionally rational allocation mechanism for any institutional IS game (parallel to Theorem 5 for physical IS games).

Having both the physical and the institutional aspects of IS formalized in the game-theoretic language, we present the aggregated IS game as the summation of the normalized form of the two games.

Definition II (IS Game). Let $G=\langle\Gamma, W\rangle$ be an IS graph, $\mathcal{G}_{P}=\langle\Gamma, W, v\rangle$ a physical IS game on $G$, and $\mathcal{G}_{I}=\langle\Gamma, W, \iota\rangle$ an institutional IS game on $G$. Then the graphical IS game is a triple $\langle\Gamma, W, \sigma\rangle$, such that for any group of firms $S \subseteq \Gamma$, the characteristic function $\sigma(S)$ is equal to $\bar{v}(S)+\bar{\iota}(S)$. 
Then, as a corollary to Propositions 9 and io and Theorem 5, we immediately deduce that any graphical IS game (from now on, IS game) preserves the properties presented in Proposition Io, hence has a non-empty core (analogous to Theorem 5 ).

Corollary 2. For any IS game $\langle\Gamma, W, \sigma\rangle$, the set of efficient and coalitionally rational allocation mechanisms is non-empty.

In other words: the normalized versions of both games satisfy the presented properties and linear aggregation preserves them.

In some application domains, one may opt for various forms of linear aggregations, i.e., to employ $\sigma=\alpha \bar{v}+\beta \bar{\iota}$ (for integer-valued positive $\alpha$ and $\beta$ ). We later highlight that due to the linearity of the allocation mechanism that we employ, our results remain valid in such a generalization of the problem.

Following the presented perspective in [Yazdanpanah et al., 2019e], an industrial symbiosis institution consists of a group of firms, a structure that specifies the outcome of collaboration among potential coalitions (in the group), and mechanism(s) responsible for coordinating the institution. Such mechanisms are basically in charge to guarantee some desirable properties in the institution. In our case-in industrial symbiosis institutions-the aim could be to ensure the stability of the institution (i.e., that no firm or group of firms has an incentive to defect the collaboratively profitable institution), the fair allocation of the collectively obtained benefits (such that the contribution of firms is reasonably reflected in their individual shares), or ideally to bring about both fairness and stability. In a general form, an industrial symbiosis institution is defined as:

Definition I2 (Industrial Symbiosis Institution). Let $\Gamma$ be a set of firms, $\mathcal{G}$ an IS game among 
firms in $\Gamma$, and $\mathfrak{M}$ a set of value allocation mechanisms. Then an industrial symbiosis institution is defined as triple $\mathcal{I}=\langle\Gamma, \mathcal{G}, \mathfrak{M}\rangle$.

In brief, this is to see an IS institution as an IS game under mechanisms in charge of distributing the collective values? ${ }^{9}$. This would be to distribute collectively obtainable benefits as well as collective operational costs for establishment and maintenance of the institution. The latter category corresponds to the focus of this chapter on allocating transaction costs in industrial symbiosis. In the next section, we present an allocation mechanism-corresponding to the notion of Shapley value and the Myerson value in graph-restricted games [Myerson, 1977; Shapley, 1953] — that satisfies both fairness and stability properties. We also elaborate on its computational complexity and tractability results.

\subsection{A Fair Allocation Mechanism for IS}

The main idea behind the fair allocation of values is to take into account the contribution of each agent to the collaborative group [Mas-Colell et al., 1995]. In industrial symbiosis management platforms, the collective transaction cost reduces to costs for establishment and maintenance of the framework—as a dynamic e-market environment ${ }^{10}$. This calls for dynamic cost allocation methods, able to grasp the physical as well as institutional nature of each agent's contribution. Roughly speaking, following an initial payment for a basic membership (to get involved in this e-market framework) it is expected that a "fair" allocation of costs for

\footnotetext{
${ }^{9}$ Note that we do not fix the allocation mechanism but take a set $\mathfrak{M}$.

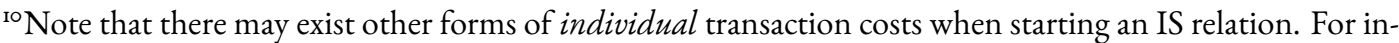
stance, some investments to calibrate the production process in order to enable accepting a waste-based resource would be categorized as a transaction cost. However, as the equipment remains property of the firm-and can be potentially used in its further relations-it is not reasonable to consider such a cost as a collective (to be shared) transaction cost.
} 
further improvements, takes into account the contribution of each participant-in terms of their role/function in the formed industrial network.

Having the game-theoretic formulation of an IS game (as an aggregation of the physical and institutional IS games), any proportion of the transaction cost that oughts to be shared among the firms-e.g., the total cost for updating the IT platform—can be distributed based on each firm's contribution to the IS game. A standard notion in computational economics, capturing the contribution of each agent in a cooperative setting, is the Shapley value [Roth and Verrecchia, 1979; Shapley, 1953]. Shapley's allocation method uniquely satisfies the so called fairness properties, which has high relevance for our domain of application in industrial organizations. In IS games, as a combination of normalized physical and institutional games among the firms, the Shapley value of a firm determines the extent of its power and influence in the institution. This value would be defined as what we call the firm's IS index.

Definition I3 (IS Index). Let $\mathcal{G}=\langle\Gamma, W, \sigma\rangle$ be an IS game. Then for any arbitrary $i \in \Gamma$, the IS index, denoted by $\Phi_{i}(\sigma)$, is equal to $\sum_{S \subseteq \Gamma \backslash\{i\}} \frac{|S| !|\Gamma|-|S|-1) !}{|\Gamma| !}(\sigma(S \cup\{i\})-\sigma(S))$.

Due to the characteristics of $\sigma$ (as a reflection of both the physical as well as institutional aspects of IS) the introduced notion of IS index is a measure that reflects the power of a firm based on its connectivity to other firms in the network and also its operational contributions by bringing about cost reductions ${ }^{\mathrm{I}}$. This index forms a basis for allocating transaction costs such that a higher contribution determines a higher share (i.e., "with great power comes great responsibility"). This approach relies on the standard rationale in cooperative cost-sharing games that agents with higher potentials ought to pay the larger share of the costs in the

"We later show that due to the graphical representation of the problem, the IS index can be formulated in a non-factorial manner. This results in lower computational complexity for calculating the IS index of each firm. 
collaborative practice [Borm et al., 200I; Marden and Wierman, 2013]. Accordingly, given an external cost function $\tau(\Gamma)$ (equal to $\tau(S)$ for all $S \subseteq \Gamma$ with $|S| \geq 2$ ), determining the (to be shared) transaction $\cos ^{\mathrm{12}}$ among the members of $\Gamma$, we present the following transaction cost allocation mechanism for IS institutions.

Definition I4 (TC Allocation for IS). Let $\mathcal{G}=\langle\Gamma, W, \sigma\rangle$ be an IS game, $\Phi_{i}(\sigma)$ the corresponding IS index for any arbitrary $i \in \Gamma$, and $\tau(\Gamma)$ a given transaction cost value for $\Gamma$. We define the cost share of agent $i \in \Gamma$ as $T_{i}(\sigma, \tau):=\frac{\Phi_{i}(\sigma) \cdot \tau(\Gamma)}{\sigma(\Gamma)}$. Allocation $T(\sigma, \tau)=$ $\left\langle a_{1}, \ldots, a_{|\Gamma|}\right\rangle$ with $a_{i}=T_{i}(\sigma, \tau)$ denotes the TC allocation for IS game $\mathcal{G}$ with respect to $\tau$.

This allocation, tailored and contextualized for the specific class of structured, graph-restricted industrial symbiosis games, (I) captures both the physical as well as the institutional aspects of this practice, (2) satisfies desirable fairness and stability properties (to be discussed in Section 4.3.I), and (3) is computationally tractable thanks to the graphical representation of the games (to be illustrated in Section 4.3.2).

\subsection{On Fairness and Stability}

Having an industrial institution, stability and fairness are two properties that can be ensured by means of well-designed mechanisms. In the case of the transaction cost allocation mechanism, stability is about (I) sharing the exact amount of the cost and (2) sharing such that no firm can benefit by defecting from the institution. On the other hand, fairness is a more

\footnotetext{
${ }^{12}$ Such a cost may consist of initial platform development costs, ongoing IT infrastructure maintenance, or extra personnel recruitment costs for updating the platform. $\tau$ is defined in a functional way merely to allow further extensions on dynamic formulations of the transaction cost function, e.g., as a temporal function of some required resources for maintaining an IS information system. In other words, $\tau(S)$ is undefined for $|S| \leq 1$ and is equal to a given value $\tau(\Gamma)$ otherwise. Note that our main question is on how to distribute this collectively defined value.
} 
complex property, concerned with (I) the symmetric contribution of firms to the institution, (2) the share of firms whose involvement are non-contributory, (3) the possibility to aggregate various institutions, and finally $(4)$ the sharing of the exact total cost. Below, we provide a formal account of these properties in axiomatic forms-based on [Mas-Colell et al., 1995] —and investigate whether they are valid in case of our suggested cost allocation mechanism.

Proposition II (Fairness Axioms). Let $\mathcal{I}=\langle\Gamma, \mathcal{G}, \mathfrak{M}\rangle$ be an industrial symbiosis institution where $\Gamma$ is the set offirms and $\mathcal{G}=\langle\Gamma, W, \sigma\rangle$ is the IS game. For any transaction $\operatorname{cost} \tau(\Gamma)>0$ we have that $\mathfrak{M}=\{T(\sigma, \tau)\}$ (Definition I4) guarantees the following fairness axioms:

(I) The collective transaction cost is efficiently allocated among the firms, formally, $\sum_{i \in \Gamma} T_{i}(\sigma, \tau)=$ $\tau(\Gamma)$ (Efficiency).

(2) The identities of the firms do not affect their share of the total transaction cost, formally, for $i, j \in \Gamma, T_{i}(\sigma, \tau)=T_{j}(\sigma, \tau)$ iffor all $S \subseteq \Gamma \backslash\{i, j\}$ we have that $\sigma(S \cup\{i\})=$ $\sigma(S \cup\{j\})$ (Symmetry).

(3) Any firm of which its contribution to any coalition is equal to its individual value, pays a transaction cost share proportional to its individual value, formally, for $i \in \Gamma$, $T_{i}(\sigma, \tau)=\frac{\sigma(\{i\}) \cdot \tau(\Gamma)}{\sigma(\Gamma)}$ if for all $S \subseteq \Gamma \backslash\{i\}$ we have that $\sigma(S \cup\{i\})=\sigma(S)+$ $\sigma(\{i\})$ (Dummy Player).

(4) For two IS games, an agent's transaction cost share in the aggregated game is equal to the summation of its share in each, formally, given an industrial game $\mathcal{G}^{\prime}=\left\langle\Gamma, W^{\prime}, \sigma^{\prime}\right\rangle$ and a corresponding transaction cost $\tau^{\prime}(\Gamma)>0$, we have that $T_{i}\left(\sigma+\sigma^{\prime}, \tau+\tau^{\prime}\right)=$ $T_{i}(\sigma, \tau)+T_{i}\left(\sigma^{\prime}, \tau^{\prime}\right)$ (Additivity). 
Proof. Our notion of IS index measures the Shapley value of each firm $i$. Following the linearity of this Shapley-based value, we have that the allocation mechanism preserves all the four properties that the Shapley value uniquely possesses [Hart, 1989].

In general, fairness and stability are orthogonal. In other words, an allocation might be fair but not stable or the other way around. Below, we present an axiomatic account of stability and show their validity for the presented transaction cost allocation method.

Proposition I2 (Stability Axioms). Let $\mathcal{I}=\langle\Gamma, \mathcal{G}, \mathfrak{M}\rangle$ be an industrial symbiosis institution where $\Gamma$ is the set offirms and $\mathcal{G}=\langle\Gamma, W, \sigma\rangle$ is the IS game. For any transaction $\cos t \tau(\Gamma)>0$ we have that $\mathfrak{M}=\{T(\sigma, \tau)\}$ (Definition I4) guarantees the following stability axioms:

(I) The collective transaction cost is efficiently allocated among the firms, formally, $\sum_{i \in \Gamma} T_{i}(\sigma, \tau)=$ $\tau(\Gamma)($ Efficiency).

(2) No subgroup faces an economic incentive to deviate from the grand coalition and benefit by paying a lower share of the transaction cost, formally, for any coalition $S \subseteq \Gamma$ with $|S| \geq 2$, we have that $\sum_{i \in S} T_{i}(\sigma, \tau) \leq \tau(S)$ (Coalitional Rationality).

Proof. The first part is valid (using Proposition II). For the second part, the current formulation of the collective transaction cost requires that $\tau(S)=\tau(\Gamma)$ which if combined with the first clause, immediately satisfies the claim ${ }^{\mathrm{r} 3}$.

Thanks to the adoption of a Shapley-based index — and its linearity property — the fairness and stability properties will be preserved in the presented aggregated form of IS institutions

\footnotetext{
${ }^{13}$ In the generalized form, where the transaction cost function is defined for all potential coalitions, the convexity of $\tau$ would be required for coalitional rationality. Note that in case such a function is available, the mere problem on "bow to distribute the collective cost among the firms" will evaporate, as its solution requires a single call to this function.
} 
and any general linear aggregation forms in which the importance of the physical and institutional contributions are weighted.

Theorem 6 (Generalizability). Let $\mathcal{I}=\langle\Gamma, \mathcal{G}, \mathfrak{M}\rangle$ be an industrial symbiosis institution where $\sigma=\alpha \bar{v}+\beta \bar{\iota}$ is the characteristic function of $\mathcal{G}$ in terms of $v$ and $\iota$, the corresponding characteristic functions in the physical and institutional IS games, respectively. We have that $\mathfrak{M}=\{T(\sigma, \tau)\}$ (Definition I4) guarantees fairness and stability in $\mathcal{I}$.

\subsubsection{Reductions and a Tractable Algorithm}

Although the presented Shapley-based IS index has the above-mentioned desirable properties, its standard formulation leads to computationally expensive algorithms. Below, we present reductions that result in an alternative formulation for computing the IS index.

Lemma 2. In a graphical physical IS game $\mathcal{G}_{P}=\langle\Gamma, W, v\rangle$, for any $i \in \Gamma$ we have that

$$
\sum_{S \subseteq \Gamma \backslash\{i\}} \frac{|S| !(|\Gamma|-|S|-1) !}{|\Gamma| !}(v(S \cup\{i\})-v(S))=\sum_{j \in \Gamma \backslash\{i\}} \frac{W_{i, j}}{2} .
$$

Proof. Based on the formulation of $v$, we have that the value of any singleton coalition $S$ is zero and for any coalition $T$ with more than two members, the value is computed based on the summation of values that bilateral relations (established within $T$ ) bring about. In other words, the average marginal contribution of any firm to any $T$ with more than two members is zero. The only set of coalitions to which a firm may have a contribution are two-member coalitions for which we have the results of [Yazdanpanah and Yazan, 20I7] that the middle point of the core corresponds to the average marginal contribution. In our graph-restricted 
games, this value is equal to the Myerson value [Myerson, 1977] and is equal to half of the summation of the values on the edges that are directly connected to $i$, i.e., $\sum_{j \in \Gamma \backslash\{i\}} \frac{W_{i, j}}{2}$.

Next, we present a reduction for computing the contributions in the institutional game.

Lemma 3. In a graphical institutional IS game $\mathcal{G}_{I}=\langle\Gamma, W, \iota\rangle$, for any $i \in \Gamma$ we have that

$$
\sum_{S \subseteq \Gamma \backslash\{i\}} \frac{|S| !(|\Gamma|-|S|-1) !}{|\Gamma| !}(\iota(S \cup\{i\})-\iota(S))=\mathfrak{C}(i) .
$$

Proof. In the institutional game, each firm $i$ 's contribution to any coalition is equal to its degree of closeness centrality. Then, the dummy player property implies the claim.

Based on these reductions we have that the transaction cost allocation is computationally tractable.

Theorem 7. Let $\mathcal{I}=\langle\Gamma, \mathcal{G}, \mathfrak{M}\rangle$ be an industrial symbiosis institution where $\Gamma$ is the set of firms and $\mathcal{G}=\langle\Gamma, W, \sigma\rangle$ is the $I S$ game. For any transaction cost $\tau(\Gamma)>0$, employing $\mathfrak{M}=\{T(\sigma, \tau)\}$ to compute the allocation $T(\sigma, \tau)=\left\langle a_{1}, \ldots, a_{|\Gamma|}\right\rangle$ is polynomial in time and space.

Proof. We present a constructive proof by providing an algorithm (see Algorithm s) that generates the allocation, of which we verify its correctness and subsequently prove the complexity claims.

Correctness: In Algorithm 5, we have that for each firm $i \in \Gamma$, the IS index $\Phi_{i}(\sigma)$ is equal to the Shapley value of $i$ in the aggregated game (of the normalized physical and institutional games). Thanks to the additivity property, this would be equal to the aggregation of Shapley 


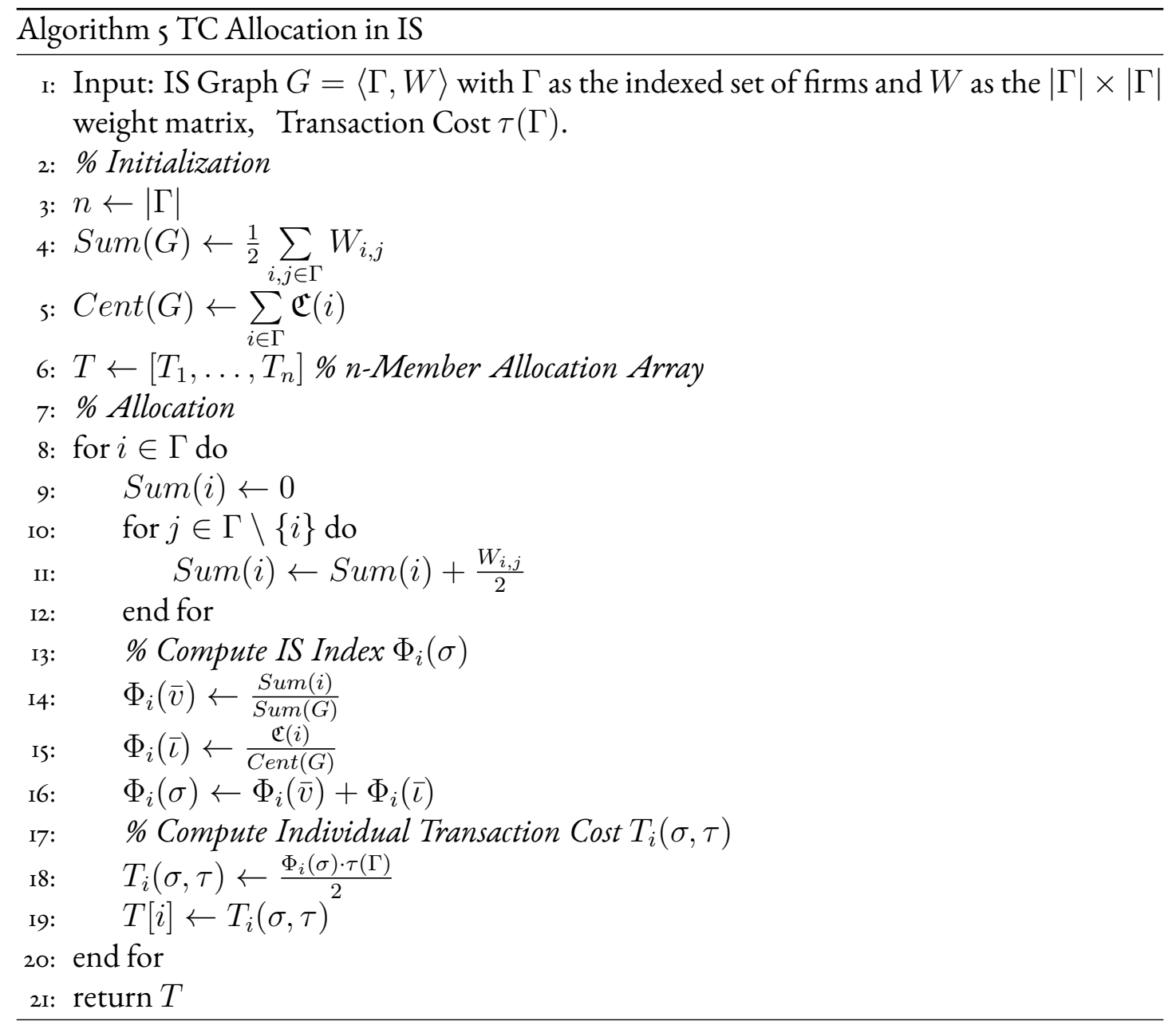


values in each game. Then, we rely on Lemma 2 and 3 for calculating the two values. Finally, for computing individual transaction costs, we have that $\sigma(\Gamma)=2$ as it is equal to $\frac{v(\Gamma)}{v(\Gamma)}+\frac{\iota(\Gamma)}{\iota(\Gamma)}$. Space Complexity: The required matrix of weights (representing the set of obtained cost reductions) is in $O\left(n^{2}\right)$ where $n$ is the size of $\Gamma$.

Time Complexity: For computing the IS indices, we have $O(n)$ on the big loop. Then in the physical game component, $\operatorname{Sum}(i)$ is in $O(n)$ (a pass on the $i$-th row in $W$ ) and $\operatorname{Sum}(G)$ is in $O\left(n^{2}\right)$ (a pass through the whole $W$ ). For the institutional part, we have that computing $\mathfrak{C}(i)$ is reducible to finding the shortest paths [Eppstein and Wang, 20oI] which is well-known to be in $O\left(n^{3}\right)$ [Floyd, 1962].

\subsection{Method Application in the Case Study}

To show the applicability of the developed method for allocating collective transaction costs among a cluster of firms $\Gamma$, we use the presented case in Example 8 and assume a total value $\tau(\Gamma)$ as the collective transaction cost, realized for an updating round in $\Gamma$ 's industrial symbiosis information system. Assuming $\tau(\Gamma)=100$ simply results in percentage calculation for individual shares.

Following the steps in Algorithm 5, we have that $\operatorname{Sum}(G)=34$ and $\operatorname{Cent}(G)=\frac{1027}{252}$. Then for each firm $i \in\{1, \ldots, 6\}$, to compute $\Phi_{i}(\bar{v})$ (as the physical component of $\Phi_{i}(\sigma)$ ), we calculate the summation of the weights on all the edges connected to $i$ and divide it by $\operatorname{Sum}(G)$. Thus we have: $\Phi_{1}(\bar{v})=\frac{7}{34}, \Phi_{2}(\bar{v})=\frac{3}{34}, \Phi_{3}(\bar{v})=\frac{4}{34}, \Phi_{4}(\bar{v})=\frac{14}{34}, \Phi_{5}(\bar{v})=$ $\frac{2}{34}, \Phi_{6}(\bar{v})=\frac{4}{34}$. For each firm $i$, adding $\frac{\mathfrak{C}(\mathfrak{i})}{\operatorname{Cent}(G)}$ to $\Phi_{i}(\bar{v})$ results in its IS index $\Phi_{i}(\sigma)=$ $\frac{13309}{34918}, \frac{4218}{17459}, \frac{9463}{34918}, \frac{11473}{17459}, \frac{3407}{17459}, \frac{4434}{17459}$ (respectively for firms 1 to 6 ). Finally, the transaction cost allocation $T$ could be generated based on $\Phi_{i}(\sigma)$. We have that: $T_{1}(\sigma, \tau)=19.06$, 
$T_{2}(\sigma, \tau)=12.08, T_{3}(\sigma, \tau)=13.55, T_{4}(\sigma, \tau)=32.86, T_{5}(\sigma, \tau)=9.76$, and $T_{6}(\sigma, \tau)=$

12.70. Note that as we employ generic graph-/game-theoretical solution concepts as a basis for the developed algorithm, our results are neither sensitive to the distribution of the cost reduction values nor to the structure of the connectivity graph.

\subsection{Practical Contributions and Open Research Directions}

Amid the institutional nature of transaction costs, to our knowledge, this work is the first proposal that translates Searle's well-established philosophy on institutional theory for the context of IS, takes it into practice for fair transaction cost allocation, and introduces a tractable algorithm for such a purpose. As a managerial decision support tool, the presented algorithm can be integrated into smart IS contracting and management frameworks to enable the automation of cost allocation procedures. For instance, as a suggested business model for IS clusters, firms would be expected to pay an initial membership fee and then be charged for further collective transaction costs based on the presented method-reflecting their operational as well as institutional contributions.

As presented, this work has immediate applicability to support IS management by means of providing a fair and stable TC allocation mechanism. In addition, it opens new research directions to study: different IS classes, various forms of TC functions, applicable notions of fairness and toolbox of mechanisms, and governance mechanisms able to observe the collective behavior of IS.

IS Classes and Graphical Constraints. While we focused on generic IS, an interesting line of research is to investigate sub-classes of IS with respect to their graphical structures. 
For instance, in most bio-based IS practices, bio-refineries are in the center of the cluster due to their crucial role as a resource treatment/recycling facility. This results in tree-like structures or in clusters of star graphs such that no two firms can implement an IS relation in the absence of a third-party refinery. This calls for tailored methods able to capture such contextual properties. To this end, tree-like graphical games [Nisan et al., 2007] would be a suggested formal foundation.

Transaction Cost Functions. An immediate extension to this work is to study transaction cost functions, formulated in terms of the operational dimensions of IS (see [Yazdanpanah et al., 2019e] for such dimensions). This results in cases where-in addition to the grand coalition-the collective transaction cost for some (but not all the) other coalitions is well-defined, through a functional formulation of TC. In such a case, we see that satisfying the coalitional rationality, by means of an allocation mechanism, calls for integrating the properties of such a function and requiring restrictions on the characteristic functions of the physical and institutional IS games.

Notions of Fairness and Toolbox of Mechanisms. We plan to relate the presented Shapley-based fair allocation to other axiomatic notions of fairness [Lan et al., 20Io]. This results in a toolbox of mechanisms, each satisfying a set of desirable properties, hence admissible in a related domain of application. Implementing such a mechanism toolbox in IS information systems enables the firm managers to opt for a relevant set of decision support services, tailored with respect to their managerial strategies and preferences. 
Norm-Aware IS Governance. Finally, a line of focus for future work is to develop governance frameworks for IS. This is to enable monitoring of the organizational behavior and enforcing normatively desirable behaviors. For such purpose, we rely on the rich literature on norm-aware coordination [Dastani et al., 2017b; Sikora and Shaw, 1998; Singh, 2013] and address open problems related to onganizational characterization of industrial symbiosis. To that end, cost allocation techniques can be employed as a financial instrument for nudging the collective behavior and to ensure the compliance of firms to contractual commitments [Anderson and Dekker, 2005]. 

Unsolved problems, that's one of the great signs of progress

in my opinion.

Kenneth Arrow

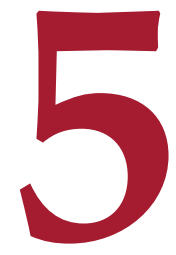

\section{Review of Contributions, Supplementary}

\section{Work and Future Research}

IN THIS CONCLUDING CHAPTER, we (I) present a summary of thesis objectives and con-

tributions, (2) review supplementary publications that either contribute to the core of this 
thesis or address related problems, (3) discuss application of the work and elaborate on the validation process, and (4) propose future research directions and open questions.

\section{I Synthesis: Revisiting Objectives and a Summary of Contributions}

Multiagent Systems (MAS) research reached a maturity to be confidently applied to real-life complex problems. Successful application of MAS methods for behavior modeling, strate-

gic reasoning, and decentralized governance, encouraged us to focus on the applicability of MAS techniques in industrial symbiosis systems and resulted in method development, integration/contextualization of techniques, and deployment of usable services in this context. In this section, we revisit the general objectives of the thesis and present a summary of contributions.

\section{I.I Revisiting Objectives}

We have directed our attention towards a form of industrial practices called Industrial Symbiosis Systems (ISS) as a highly dynamic domain of application for MAS techniques. In ISS, firms aim to reduce their material and energy footprint by circulating reusable resources among the members. To enable systematic reasoning about ISS behavior and support firms' (as well as ISS designers') decisions, we linked industrial engineering with multiagent systems research. In this view, the objective was to support industrial symbiosis decision processes by introducing $(\mathrm{I})$ representation and reasoning frameworks to enable a systematic evaluation of industrial symbiosis opportunities, (2) coordination and control techniques to foster the implementation of desirable industrial symbiosis relations, and (3) cost allocation methods that capture institutional as well as economic contributions of firms in an implemented industrial 
symbiosis network.

Each of the three above-mentioned goals were the main focus of a chapter in this thesis. In chapter 2, we focused on the industrial symbiosis evaluation, in Chapter 3 coordination was the focal point, and Chapter 4 aimed for a cost allocation method tailored for industrial symbiosis. Below, we review the contribution of each chapter to see whether the achieved outcomes meet the envisaged objectives.

\section{I.2 Summary of Contributions}

Evaluating Industrial Symbiosis Opportunities (Chapter 2): The main contribution of this chapter is a formal decision support method that takes into account operational and epistemic aspects for filtering industrial symbiosis opportunities. Using this, firms can evaluate various opportunities and see a spectrum of most-promising to least-promising cases. Moreover, to address potential epistemic limitations, we developed a method that employs firms' distributed knowledge and enables learning about all the mutually promising relations. In addition to the algorithmic account of these two methods, we introduced novel concepts for reasoning about industrial symbiotic relations.

Coordinating Industrial Symbiosis Networks (Chapter 3): This chapter provides a coordinated multiagent framework for implementing ISNs. It presents algorithms to generate regulatory rules for ensuring the implementability of desirable symbiotic collaborations. In practice, such a framework can be used by policy-makers aiming to foster socioeconomically desirable symbiotic collaborations. 
Allocating Transaction Costs in Industrial Symbiosis (Chapter 4): This chapter proposes a translation of Searle's account of institutional economics in the context of industrial symbiosis and introduces a tractable algorithm for fair transaction cost allocation in industrial symbiosis networks. As a managerial decision support tool, the presented algorithm can be integrated into smart IS contracting and management frameworks to enable the automation of cost allocation procedures.

Multiagent Industrial Symbiosis Systems: In general, this dissertation on Multiagent Industrial Symbiosis Systems is an attempt to address evaluation, coordination, and cost-allocation as three decision-making aspects—to realize industrial symbiosis-that require decision support tools. Through the course of this PhD project, we also focused on other dimensions of the topic (presented in Section 5.2). However, there are still various interesting aspects of the topic, each revealing open research problems to study.

\subsection{Supplementary Research}

This thesis benefited from supplementary publications: on cooperative and coordinated industrial symbiosis games [Yazdanpanah and Yazan, 20I7; Yazdanpanah et al., 20I8], on normaware industrial symbiosis [Yazdanpanah et al., 2016, 2019b], on emergence and evolution of fair industrial symbiosis [Yazan et al., 2017a, 2019], on requirements for agent-based industrial symbiosis modeling [Fraccascia et al., 2019], and on energy-based industrial symbiosis [Fraccascia et al., 2019]. In the following, we elaborate on this body of work. Some parts directly contribute to the core of the thesis while others address related research questions-raised through the course of the $\mathrm{PhD}$ research. 


\subsection{Cooperative and Coordinated Industrial Symbiosis Games}

The research work presented in [Yazdanpanah and Yazan, 2017; Yazdanpanah et al., 2018] introduces a formal account of industrial symbiosis using cooperative game theory. In [Yazdanpanah and Yazan, 20I7], the focus is on two-player games while [Yazdanpanah et al., 20I8] discusses the multi-player case.

Industrial Symbiotic Relations as Cooperative Games [Yazdanpanah and Yazan, 2017]: In this work, we introduce a game-theoretical formulation for a specific form of collaborative industrial relations called "Industrial Symbiotic Relation (ISR) games" and provide a formal framework to model, verify, and support collaboration decisions in this new class of two-person operational games. ISR games are formalized as cooperative cost-allocation games with the aim to allocate the total ISR-related operational cost to involved industrial firms in a fair and stable manner by taking into account their contribution to the total traditional ISR-related cost. We tailor two types of allocation mechanisms that firms can use to implement cost allocations that result in a collaboration that satisfies the fairness and stability properties. Moreover, while industries receive a particular ISR proposal, our introduced methodology is applicable as a managerial decision support tool to systematically verify the quality of the ISR in question and check whether the implemented allocation mechanism is stable and fair. The results of [Yazdanpanah and Yazan, 20I7] are used as fair and stable cost allocation mechanisms in Chapter 2.

Industrial Symbiotic Networks as Coordinated Games [Yazdanpanah et al., 20I8]: This paper reports an overview of the presented work in Chapter 3. It discusses the idea 
and requirements for implementing a specific form of collaborative industrial practicescalled Industrial Symbiotic Networks (ISNs) —as MC-Net cooperative games and addresses the so called ISN implementation problem. This is, the characteristics of ISNs may lead to inapplicability of fair and stable benefit allocation methods even if the collaboration is a collectively desired one. Inspired by realistic ISN scenarios and the literature on normative multiagent systems, it uses regulations and normative socioeconomic policies as coordinative elements for ISN games.

\subsubsection{Normative Industrial Symbiosis}

The presented research in [Yazdanpanah et al., 2016, 2019b] aims to capture the normativity of industrial symbiosis and proposes a norm-aware perspective for governing industrial symbiosis. These papers relate to the presented approach in Chapter 3 as they suggest a normbased perspective but are methodologically distinguishable as they are based on commitmentbased organizational models [Dastani et al., 20I7b; Telang and Singh, 20I2]. Moreover, they correspond to the presented approach in Chapter 4 as they apply Searle's perspective on institutional economics.

Normative Industrial Symbiotic Networks: A Position Paper [Yazdanpanah et al., 20I6]: In this position paper, we introduce a normative, multi-agent perspective on the field of industrial symbiosis research and propose normative institutions as a key technology for operating Industrial Symbiotic Networks (ISNs), both as a framework to represent and reason about dynamic behaviour of ISNs and as a platform for design and maintenance of such networks. We discuss the requirements of normative agent-based frameworks for ISNs with respect to agent interactions, joint commitments, and the organization to monitor in- 
teractions in ISNs. A comprehensive account of the presented norm-aware perspective in [Yazdanpanah et al., 2016] appears in [Yazdanpanah et al., 2019b].

Self-Governing Industrial Symbiosis [Yazdanpanah et al., 20I9b]: In this paper, we develop a normative multiagent framework for self-governing Industrial Symbiosis (IS) systems. In contrast to traditional centrally-managed approaches that aim for an equilibriumoriented optimality, the proposed framework captures behavioral, institutional, and normative aspects of IS. To achieve this, we introduce operational agent interaction protocols, tailor a model of joint commitment dynamics, and introduce interaction monitoring mechanisms for IS. Such a framework can be the basis for implementing decentralized administration technologies and smart contracting systems able to observe the behavior of IS systems and for governing the evolution of commitments.

\subsubsection{Emergence and Evolution of Fair Industrial Symbiosis}

The work presented in [Yazan et al., 2017a, 2019] is focused on the dynamics of collaborative (in contrast to opportunistic) strategies in industrial symbiosis relations. Preliminary results are presented in [Yazan et al., 20I7a] while [Yazan et al., 2019] addresses the topic in a com-

prehensive manner. On the one hand, this work relates to the body of the thesis as it employs game-theoretical concepts to study the emergence of collaborative strategies. On the other hand, it is complementary due to the use of methods from enterprise input-output analysis as well as simulation-based techniques for modeling the evolution of the relations.

Cooperation Decisions in Industrial Symbiotic Relations ～[Yazan et al., 2Oi7a]: This work focuses on dynamics of benefit-sharing in industrial symbiosis (IS) relations as a 
determinator of firms' business strategy in such relations. As a cooperative industrial practice, IS is expected to play a major role in implementing the circular economy paradigm. Firms involved in Industrial Symbiotic Relations (ISRs), i.e.,emerging relations for waste exchanges among production processes of distinct firms, can enjoy mutual environmental and economic benefits. Moreover, ISRs have a positive influence on both the resilience of firms and the efficiency in exploiting available resources. Although IS takes place between the production processes of firms, it makes indirect and induced impacts on the physical and monetary flows through their traditional supply chains. Hence, firms need analytical tools tailored to support their decisions whether to cooperate in, and operationalize a particular ISR proposal measuring such impacts. Such a decision support tool has to regard multiple operational aspects like the presence of competitors and regulations. Although methods for analyzing each of these dimensions separately exist, analyzing the cooperation decisions in ISRs calls for joint methods able to take into account multiple aspects. To this end, we apply Enterprise Input-Output (EIO) analysis for modeling the physical flows of resources and exploit game-theoretic schemes for reasoning about cooperation decisions. Moreover, we study the macro-behavior of ISRs regarding the cooperation decisions using multiagent-based simulations. Our empirical context is based on a circular business case from the process industry. Our study provides practical insights for firm managers facing the cooperation decisions in potential ISRs. Furthermore, a methodological advance in studying IS is provided by bridging input-output analysis, game theory, and agent-based simulation.

Strategic Cooperative Behavior in Industrial Symbiosis: A Game-Theoretical Approach Integrated with Agent-Based Simulation [Yazan et al., 20i9]: This paper investigates the competition phase of industrial symbiosis relationships, where 
companies exchange wastes for inputs and negotiate how to share the additional costs to operate the waste exchange. In our game-theoretical model two strategies that companies can adopt for sharing costs are considered: a fair strategy and an opportunistic strategy. Then, an agent-based model is used to simulate the game iterated over time and to investigate how the agents (firms) adapt their strategies according to their experience and observing the actions of their counterparts in previous periods. Simulation results show that agents learn that applying the fair strategy is beneficial in the long run, despite the fact that in the short period they can gain more by following the opportunistic strategy.

\subsubsection{Requirements for Agent-Based Industrial Symbiosis Modeling}

A Framework of Industrial Symbiosis Systems for Agent-based Simulation [Fraccascia et al., 2019]: In this work, we review the requirements for agent-based Industrial Symbiosis (IS) modeling by performing a literature review on drivers and barriers for implementing industrial symbiosis and on actors involved in such a process. The Agent-Based Modeling $(\mathrm{ABM})$ approach has been recently adopted to advance the knowledge in the IS field, in particular to explore the extent to which the establishment of IS relationships can be affected by policy measures, benefit-sharing contracts, information-sharing tools, or social factors. The agent-based models proposed in the literature are mostly case-specific and in some cases contradict one another in terms of agents considered crucial, their key attributes, available actions, and decisional rules. The aim of this paper is to propose a generic framework on how to model IS systems with ABM in a comprehensive way. The paper provides a literature review on IS to identify the set of involved agents in IS, their attributes, and their potential actions. Based on the results of the literature review, a general framework is pro- 
posed and discussed. The outcome of this work is complementary to the thesis as it provides a systematic method for adoption of agent-based simulation in the context of IS modeling.

\subsubsection{ENERgy-BASEd INDUStrial Symbiosis}

Energy-based Industrial Symbiosis: A Literature Review for Circular EnERgy Transition [Fraccascia et al., 2019]: This paper provides a systematic literature review on approaches for energy-based industrial symbiosis (IS), i.e., IS synergies aimed at reducing the amount of energy requirement from outside industrial systems or the amount of traditional fuels used for energy production. Energy-based IS cases are identified among 682 papers published between 1997 and 2018. Three categories of symbiotic synergies are discussed: (I) energy cascade, (2) fuel replacement, and (3) bio-energy production. Through the review, different strategies to implement energy-based IS synergies are identified for each of the above-mentioned categories. Furthermore, drivers, barriers, and enablers of business development in energy-based IS are highlighted from a technical, economic, regulatory, and institutional perspective. Accordingly, future research directions are recommended. This work is complementary to the thesis as it presents insights on a specific class of industrial symbiosis systems by discussing aspects related to IS practices focused on energy. One way to use the results of this work is to define well-justified behavioral rules for energy-specific agent-based IS models. In such a model, the set of drivers, barriers, and enablers (for each category of agents) can be the basis for rules that express how that type of agent acts-e.g., seeing the set of drivers and in response to changes in the environment (reactive behavioral rules) or considering the enablers and in pursue of its motives/preferences (proactive behavioral rules). 


\subsection{Application and Validation Process}

In this section we first elaborate on the application of two decision support tools-resulting from this PhD research - in the SHAREBOX project [SHAREBOX, 2019]. Secondly, we expand on the validation process. To respect SHAREBOX's grant agreement, non-disclosure agreements and other confidentiality concerns, we change the name of the decision support tools and generally refer to them as TOE (an IS decision support tool for opportunity evaluation) and TOC (an IS decision support tool for cost allocation). Moreover, we avoid mentioning the name of involved parties in validation workshops, omit exact dates, and merely refer to the general insights we gained through the process. ${ }^{\mathrm{I}}$ In a nutshell, we can highlight that the two decisions support services are preliminarily evaluated as "with commercialization potential”.

\subsection{Application: From Decision Support Algorithms to Implemented Tools}

The main focus of the developed tools is on (I) the evaluation phase of industrial symbiosis (i.e., when potential symbiotic partners are identified and firms seek to know about a ranking of the options) and (2) the implementation phase (i.e., when firms have selected a partner and are going to establish a feasible contract). For the former, we developed $T O E$ while the latter is addressed by $T O C$.

TOE: As discussed in Chapter 2, evaluating industrial symbiosis opportunities can be a complex task considering that each relation can be implemented in various ways (e.g., using

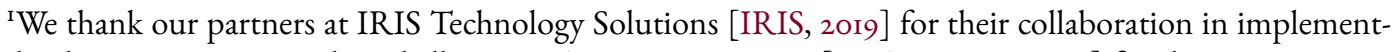
ing the decision support tools and all our SHAREBOX partners [SHAREBOX, 20I9] for their constructive comments.
} 
different modes of transportation or recycling/treatment technologies). Based on the results of [Yazdanpanah et al., 20I9e], we developed TOE, a practical decision support algorithm that allows firms to compare potential implementations of a relation.

TOC: For the implementation phase of industrial symbiosis-where operational aspects such as the allocation of costs/benefits are the main concern-we developed TOC. This tool follows the presented cost allocation techniques in [Yazdanpanah and Yazan, 2017] which in turn rely on standard solution concepts in computational economics. Using this tool, firms receive support on a cost/benefit allocation that reflects their contributions to the collective cost/benefit.

\subsubsection{Validation: From Naive Tools to Usable Services}

The two decision support tools were presented to and scrutinized by SHAREBOX partnersfrom industry and academia-in various validation workshops. The outcome of validation workshops and the validation process as a whole resulted in (I) having a better understanding of the needs of different users and developing various modes that suit their requirements, (2) improvements in usability of the services, and (3) addressing privacy-related concerns of firms as the main group of our final users.

Different Modes for Different Users: As a result of the feedback we received on earlier versions of the tools, we realized that some firms like to use the tools individually (e.g., in order to have some pre-negotiation support) while others are interested in using them together with their potential partners. Considering this, we integrated new modes into both tools. Although the basis of the algorithms remained untouched, adding new modes and 
differentiating on the number of users that provide information to the tools resulted in new functionalities.

ImProving Usability AND Privacy: Having a minimum viable product, users had the chance to test the tools and check how they perform in extreme cases (e.g., by entering out of range values and checking the results). As a result, we received comments on the lack of clarity and also notes on whether the privacy is respected when firms use the tools with a partner. To improve the usability, we introduced brief manuals, step-by-step instructions to guide the user, and mouse-over helps on the type of expected input/output for each tool. To respect privacy-related concerns, we implemented a mechanism that allows users to collaborate during the data-entry process but prevents them to access the private bits of the data.

\subsection{Future Research Directions}

In this dissertation, we focused on multiagent decision support tools for industrial symbiosis systems (ISS). Below, we elaborate on some open problems and highlight the relation to relevant multiagent techniques ${ }^{2}$.

InCENTIVE EngINEERING: To support the implementation of an ISS-with the aim to guarantee its resilience and stability-legislative bodies may introduce external support in terms of monetary incentives. This is either by providing subsidies to support socio-environmentally desirable ISS implementations or by imposing taxes to suppress undesirable practices. To

\footnotetext{
${ }^{2}$ The set of presented research directions in this section is mainly based on "Vahid Yazdanpanah, Devrim Murat Yazan, Jos van Hillegersberg, and Mehdi Dastani. An introduction to engineering multiagent industrial symbiosis systems: Potentials and challenges. Presented at 7 th International Workshop on Engineering Multi-Agent Systems (EMAS 2019), Montreal, Canada, I3th-I4th May, 2019a" [Yazdanpanah et al., 2019a, Section 3].
} 
have a successful allocation of incentives, we lack policy-support tools, aware of the structure and dynamics of ISS. To tackle this problem ${ }^{3}$, we see that involved firms can be modeled as resource-controllers in an ISS game. This approach enables the applicability of incentive engineering methods for ensuring properties in boolean games [Wooldridge et al., 2013].

GOVERNANCE: In an ISS, firms will get involved in commitments (at the institutional level) and can affect the state of resources (at the physical level). To be able to specify, monitor, and maintain a well-behaving ISS, we need multiagent commitment governance frameworks and interorganizational communication protocols. Following [Singh, 2013], we see norms as a basis for "administration of stakeholders by stakeholders" and for offering coordination as a service [van Hillegersberg et al., 2OI2] in ISS. While we elaborated on norm-based coordination approaches, specifying ISS communication languages, identifying their properties, and developing norm-based governance platforms for ISS are still open problems. Moreover, ISS are expected to be long-term and to evolve over time. Then to capture their dynamics over time and accordingly update the governance models, participation and feedback of industrial symbiosis practitioners are among the key requirements. For such a purpose, integrating participatory policy analysis models [Mehryar et al., 2017] with norm-aware models [Alechina

\footnotetext{
${ }^{3}$ It is noteworthy that incentive engineering for supporting IS - and environment-friendly industrial practices in general-is not only of importance in academia but also has extreme practical significance; as legislative bodies are often struggling with developing supportive policies in this context. Such policies are not only to cover tangible operational costs but should also address the intangible external costs. For instance, costs and damages related to resource depletion, hazardous particle emission, and other forms of environmental pollution that are proved to be among the causes for global warming. Note that such emissions in the end translate in (often substantial) costs to mitigate the consequences, and that are partly costs legislative bodies in the long run will face. This shows the practical relevance of the topic and its clear links to methods for addressing the drivers behind global warming [Poschen, 2017; Wysokińska, 2016], to the body of work on climate change mitigation policies [Michaelowa and Michaelowa, 2017; Newell, 2010], and to analytical tools for securing policy effectiveness [Filatova et al., 2009; Mehryar et al., 2017, 2019]. This calls for a long-horizon approach in which the governments (and in the end, the public) are one of the game players.
} 
et al., 20I3; Kaponis and Pitt, 2006; Tinnemeier et al., 20Io] are a welcome direction for further research.

Organizational Characterization: For design and coordination of ISS, a critical point is to understand, model, and reason about the dynamics of roles, relationships, and authority structures. For example, in some forms of ISS the role of facilitating firms (i.e., broker agents) is crucial while in others, resource recycling plants play a key role. In our proposed ISS model, we simply reason about action-based dynamics of ISS, thus abstract from organizational structures and notions such as roles, obligations, permissions, and prohibitions. However, in complex multi-agent ISS scenarios, it is more natural to introduce high-level organizational notions (I) for capturing the complex dynamics of ISS and (2) for coordinating its behavior accordingly. This is to move from "ISS as a multiagent system" to "ISS as a multiagent organization". To this end, one may develop a mapping between multiagent organizational paradigms [Horling and Lesser, 2004] and different forms of ISS. This calls for identifying the set of roles in various forms of ISS - with respect to firms' control on resources and their strategic position - and accordingly verifying the appropriateness of organizational structures. For instance, while hierarchical structures seem applicable for material-based ISS (due to geographical proximity of firms in an industrial cluster), a coalition-based structure might be appropriate for service-based ISS (due to involvement of potentially distant clusters).

Social Aspects, Trust, and Openness: While an ISS designer provides a secure platform (to nurture stable relations), the stability of relations also depends of the dynamics of trust and firms' socially-bounded autonomy-in the sense of [Conte and Castelfranchi, 1995]. 
Note that in ISS, we are dealing with (semi)autonomous firm managers who act with respect to (not only) the economic analysis on obtainable benefits (but also) the set of subjective preferences - that may be affected by social practices or trust dynamics [Dignum et al., 2015]. Thus, capturing agents' preferences and trust in reasoning about the behavior of ISS might be an interesting attempt for applying available trust formalizations [Huynh et al., 2006; Pinyol and Sabater-Mir, 2013; Wang and Singh, 2007] in a practical domain. A practical way forward would be to capture firms' subjective preferences-on the list of potential symbiotic coalitions - and employ it as a filtering measure on the feasibility of economically profitable instances [Yazdanpanah et al., 2019e].

Finally, while most frameworks for modeling organizations focus on a closed system, there is a lack of open multiagent models to specify ISS ${ }^{4}$. Following [Dignum et al., 2007; Ostrom, 2015], we believe that allowing the ability to leave/join an ISS (as an industrial institution) calls for a new form of dynamic stability/resilience analysis. To capture openness in ISS, one may employ financial incentives [Kumar and van Hillegersberg, 200o], use social bounds [Huynh et al., 2006], or apply expectation-aware methods [Klein et al., 2003].

\footnotetext{
${ }^{4}$ Formal modeling of open MAS is still an open problem. This motivates limiting the attention to a specific class, e.g., multiagent ISS, and aiming to tackle the problem under its boundaries. For instance, in an established ISS, partners have often jointly invested in the set-up of the network. Then, if new partners want to join, without the burden of the exploration of the many possibilities (because they can learn from, and often are motivated by, the existing practice) it may be relevant to investigate whether new-comers need to pay some extra "entrance fee". Similarly, the leave of a firm may damage the remaining partners and again the question is whether a penalty should be in place. As elaborated, openness in IS has links to coordination methods and to incentive/penalty schemes for governing the behavior of ISSs.
} 



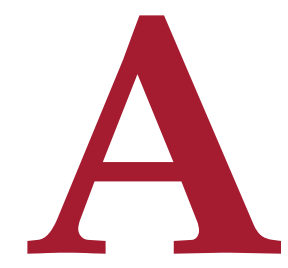

\section{Shapley-Based Cost Sharing for ISRs}

IN THIS APPENDIX, we present a brief mathematical derivation, as well as the intuition behind the Shapley-based cost sharing for IS (in Chapter 2).

Consider two firms $A$ and $B$ and suppose that implementing the ISR leads to 3 T operational costs $O$. Also, suppose that $T_{A}$ and $T_{B}$ were the traditional costs for $A$, respectively $B$. 
Obviously, we assume that $O<T_{A}+T_{B}$ otherwise there would not be any deal.

Cost sharing of $O$ means that $A$ is taking a part $\beta O$ of $O$ and similarly $B$ is taking $(1-$ $\beta) O$. Then-considering economic rationality-players are only willing to accept their share if $\beta O<T_{A}$ and $(1-\beta) O<T_{B}$ which together gives $\frac{1-T_{B}}{O}<\beta<\frac{T_{A}}{O}$. Such a $\beta$ only exists if $\frac{1-T_{B}}{O}<\frac{T_{A}}{O}$ which is equivalent to $O<T_{A}+T_{B}$ which was our premise, so there indeed is a choice of $\beta$ values. This relates to what [Yazdanpanah and Yazan, 2017] shows as the non-emptiness of the core and stability of a cost allocation.

Then, roughly speaking, what the Shapley value defines as a fair cost allocation in this form of two-person game is taking the $\beta$ value halfway the two extremes, i.e.,

$$
\beta=\frac{1}{2}\left(1-\frac{T_{B}}{O}\right)+\frac{1}{2} \frac{T_{A}}{O}=\frac{1}{2}\left(\frac{O+T_{A}-T B}{O}\right)
$$

leading to a fair cost share for firm $A$ of $\beta O=\frac{1}{2}\left(O+T_{A}-T_{B}\right)$ and a fair cost share for $B$ equal to $(1-\beta) O=\frac{1}{2}\left(O+T_{B}-T_{A}\right)$ which is the formula we show in Section 2.3.3 (based on [Yazdanpanah and Yazan, 2017]).

The intuitive reasoning of is that, if $A$ had the larger traditional costs, it is reasonable that she also takes a larger part (than $B$ ) of the new (reduced) costs, and vice versa. 


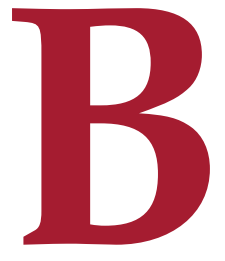

\section{Balancedness of ISN ${ }_{\Lambda}$}

IN THIS APPENDIX, we present an alternative proof for Lemma I. In this proof, we avoid going through the notion of supermodularity by merely building on the definition of balancedeness and using the superadditivity of $\mathrm{ISN}_{\Lambda}$.

Denote the two firms by $a$ and $b$, and hence the grand coalition is $\{a, b\}$. Note that the 
definition of a balanced vector basically applies to all sets (of players), except to the empty set.

Now, a balanced vector $\left(\lambda_{a}, \lambda_{b}, \lambda_{\{a, b\}}\right)$ satisfies $\lambda_{a}+\lambda_{\{a, b\}}=1$ and $\lambda_{b}+\lambda_{\{a, b\}}=1$.

The game is balanced (apply the definition) if such a vector satisfies:

$$
\lambda_{a} v(a)+\lambda_{b} v(b)+\lambda_{\{a, b\}} v(\{a, b\}) \leq v(\{a, b\})
$$

which follows from

$$
\begin{gathered}
\lambda_{a} v(a)+\lambda_{b} v(b)+\lambda_{\{a, b\}} v(\{a, b\}) \\
\leq\left(1-\lambda_{\{a, b\}}\right) v(a)+\left(1-\lambda_{\{a, b\}}\right) v(b)+\lambda_{\{a, b\}} v(\{a, b\}) \\
\leq\left(1-\lambda_{\{a, b\}}\right)(v(a)+v(b))+\lambda_{\{a, b\}} v(\{a, b\}) \\
\leq\left(1-\lambda_{\{a, b\}}\right) v(\{a, b\})+\lambda_{\{a, b\}} v(\{a, b\})=v(\{a, b\})
\end{gathered}
$$

where in the last inequality we used superadditivity, i.e., $v(a)+v(b) \leq v(\{a, b\})$. 


\section{References}

Atila Abdulkadiroğlu, Parag A. Pathak, and Alvin E. Roth. The new york city high school match. American Economic Review, pages 364-367, 2005.

Thomas Ågotnes, Valentin Goranko, Wojciech Jamroga, and Michael Wooldridge. Knowledge and ability. In Hans van Ditmarsch, Joseph Halpern, Wiebe van der Hoek, and Barteld Kooi, editors, Handbook of Epistemic Logic, pages 543-589. College Publications, 2015.

Vito Albino, Rosa Maria Dangelico, Angelo Natalicchio, and Devrim Murat Yazan. Alternative energy sources in cement manufacturing. Network for Business Sustainability, 2011.

Vito Albino, Luca Fraccascia, and Ilaria Giannoccaro. Exploring the role of contracts to support the emergence of self-organized industrial symbiosis networks: an agent-based simulation study. Journal of Cleaner Production, II2:4353-4366, 2016.

Huib Aldewereld, Frank Dignum, Andrés García-Camino, Pablo Noriega, Juan Antonio Rodríguez-Aguilar, and Carles Sierra. Operationalisation of norms for electronic institutions. In Coordination, Organizations, Institutions, and Norms in Agent Systems II, pages 163-176. Springer, 2007.

Natasha Alechina, Mehdi Dastani, and Brian Logan. Reasoning about normative update. In Proceedings of IJCAI 2013, pages 20-26, 2013.

Rajeev Alur, Thomas A. Henzinger, and Orna Kupferman. Alternating-time temporal logic. Journal of the ACM (JACM), 49(5):672-713, 2002.

Mikael Skou Andersen. An introductory note on the environmental economics of the circular economy. Sustainability Science, 2(I):133-I40, 2007.

Shannon W. Anderson and Henri C. Dekker. Management control for market transactions: The relation between transaction characteristics, incomplete contract design, and subsequent performance. Management Science, 5I(I2):I734-I752, 2005. 
Viknesh Andiappan, Raymond R. Tan, and Denny K.S. Ng. An optimization-based negotiation framework for energy systems in an eco-industrial park. Journal of cleaner production, I29:496-507, 2016.

Clinton J. Andrews. Building a micro foundation for industrial ecology. Journal of Industrial Ecology, 4(3):35-51, 2000.

Giulia Andrighetto, Guido Governatori, Pablo Noriega, and Leendert van der Torre. Normative multi-agent systems, volume 4. Schloss Dagstuhl-Leibniz-Zentrum fuer Informatik, 2013.

David W. Ash. Empowering automated trading in multi-agent environments. Computational Intelligence, 20(4):562-583, 2004.

Matteo Baldoni, Cristina Baroglio, and Elisa Marengo. Behavior-oriented commitmentbased protocols. In ECAI, volume 215, pages 137-I42, 2010.

Jay B. Barney and William Hesterly. Organizational economics: Understanding the relationship between organizations and economic analysis. The $S A G E$ bandbook of organization studies, pages III-I48, 2006.

Alex Bavelas. Communication patterns in task-oriented groups. The Journal of the Acoustical Society of America, 22(6):725-730, 1950.

Maria Bengtsson and Sören Kock. Coopetition in business networks: to cooperate and compete simultaneously. Industrial marketing management, 29(5):4II-426, 2000.

Rene Van Berkel, Tsuyoshi Fujita, Shizuka Hashimoto, and Minoru Fujii. Quantitative assessment of urban and industrial symbiosis in kawasaki, japan, 2009.

Louis J. Billera and David C. Heath. Allocation of shared costs: A set of axioms yielding a unique procedure. Mathematics of Operations Research, 7(I):32-39, 1982.

Olivier Boissier and M. Birna Van Riemsdijk. Organisational reasoning agents. In Agreement Technologies, pages 309-320. Springer, 2013.

Olga N. Bondareva. Some applications of linear programming methods to the theory of cooperative games. Problemy kibernetiki, I0:I19-139, 1963.

Frank Boons and Leo W. Baas. Types of industrial ecology: the problem of coordination. Journal of cleaner production, 5(I-2):79-86, 1997. 
Rafael H. Bordini, Lars Braubach, Mehdi Dastani, Amal El Fallah-Seghrouchni, Jorge J. Gómez-Sanz, João Leite, Gregory M. P. O’Hare, Alexander Pokahr, and Alessandro Ricci. A survey of programming languages and platforms for multi-agent systems. Informatica (Slovenia), 30(I):33-44, 2006.

Peter Borm, Herbert Hamers, and Ruud Hendrickx. Operations research games: A survey. Top, 9(2):I39, 2001.

Lee Burke and Jeanne M. Logsdon. How corporate social responsibility pays off. Long range planning, 29(4):495-502, 1996.

Stefan Bussmann, Nicolas R. Jennings, and Michael Wooldridge. Multiagent systems for manufacturing control: a design methodology. Springer Science \& Business Media, 2013.

Alberta C. Carpenter and Kevin H. Gardner. Use of industrial by-products in urban transportation infrastructure: argument for increased industrial symbiosis. In Electronics and the Environment, 2008. ISEE 2008. IEEE International Symposium on, pages I-7. IEEE, 2008.

Jonathan Carter, Elijah Bitting, and Ali A. Ghorbani. Reputation formalization for an information-sharing multi-agent system. Computational Intelligence, I8(4):515-534, 2002.

Georgios Chalkiadakis, Edith Elkind, and Michael Wooldridge. Computational aspects of cooperative game theory. Synthesis Lectures on Artificial Intelligence and Machine Learning, 5(6):I-168, 2 OII.

Xudong Chen, Tsuyoshi Fujita, Satoshi Ohnishi, Minoru Fujii, and Yong Geng. The impact of scale, recycling boundary, and type of waste on symbiosis and recycling. Journal of Industrial Ecology, I6(I):I29-I4I, 2012.

Marian Chertow. Industrial symbiosis: literature and taxonomy. Annual review of energy and the environment, 25(I):313-337, 2000.

Marian Chertow. Uncovering industrial symbiosis. Journal of Industrial Ecology, II(I):II-30, 2007.

Marian Chertow and John Ehrenfeld. Organizing self-organizing systems: Toward a theory of industrial symbiosis. Journal of industrial ecology, I6(I):I3-27, 2012.

Irene Mei Leng Chew, Raymond R. Tan, Dominic Chwan Yee Foo, and Anthony Shun Fung Chiu. Game theory approach to the analysis of inter-plant water integration in an eco-industrial park. Journal of Cleaner Production, I7(I8):I6II-I6I9, 2009. 
Shauhrat S. Chopra and Vikas Khanna. Understanding resilience in industrial symbiosis networks: Insights from network analysis. Journal of environmental management, I4I:8694, 2014 .

Rosaria Conte and Cristiano Castelfranchi. Cognitive and social action. Taylor \& Francis, 1995.

Inês Costa, Guillaume Massard, and Abhishek Agarwal. Waste management policies for industrial symbiosis development: case studies in european countries. Journal of Cleaner Production, 18(8):815-822, 2010.

Natalia Criado, Estefania Argente, Vicente Julián, and V. Botti. Designing virtual organizations. In 7 th International Conference on Practical Applications of Agents and Multi-Agent Systems (PAAMS 2009), pages 440-449. Springer, 2009.

Carl J. Dahlman. The problem of externality. The journal of law and economics, 22(I): I4I-I62, I979.

Mehdi Dastani, Sebastian Sardiña, and Vahid Yazdanpanah. Norm enforcement as supervisory control. In PRIMA 2017: Principles and Practice of Multi-Agent Systems - 2oth International Conference, Nice, France, October 30 - November 3, 20I7, Proceedings, pages 330-348, $2017 \mathrm{a}$.

Mehdi Dastani, Leendert van der Torre, and Neil Yorke-Smith. Commitments and interaction norms in organisations. Autonomous Agents and Multi-Agent Systems, pages I-43, 2oI7b.

Xiaotie Deng and Christos H. Papadimitriou. On the complexity of cooperative solution concepts. Mathematics of Operations Research, 19(2):257-266, 1994.

Pierre Desrochers. Industrial symbiosis: the case for market coordination. Journal of Cleaner Production, I2(8):I099-IIIO, 2004.

Frank Dignum. Review of non-equilibrium social science and policy: Introduction and essays on new and changing paradigms in socio-economic thinking (understanding complex systems). Journal of Artificial Societies and Social Simulation, 2018.

Frank Dignum, Virginia Dignum, John Thangarajah, Lin Padgham, and Michael Winikoff. Open agent systems??? In International AOSE Workshop, pages 73-87, 2007. 
Frank Dignum, Rui Prada, and Gert Jan Hofstede. From autistic to social agents. In Proceedings of the 2014 international conference on Autonomous agents and multi-agent systems, pages II6I-II64. International Foundation for Autonomous Agents and Multiagent Systems, 20I4.

Frank Dignum, Virginia Dignum, Rui Prada, and Catholijn M. Jonker. A conceptual architecture for social deliberation in multi-agent organizations. Multiagent and Grid Systems, II(3):I47-166, 20I5.

Theo S.H. Driessen. Cooperative games, solutions and applications, volume 3. Springer Science \& Business Media, 2013.

David Eppstein and Joseph Wang. Fast approximation of centrality. In Proceedings of the twelfth annual ACM-SIAM symposium on Discrete algorithms, pages 228-229. Society for Industrial and Applied Mathematics, 200I.

Daniel C. Esty and Michael E. Porter. Industrial ecology and competitiveness. Journal of Industrial Ecology, 2(I):35-43, 1998.

Ronald Fagin, Joseph Halpern, Yoram Moses, and Moshe Vardi. Reasoning about knowledge. MIT press, I995.

Tatiana Filatova, Dawn Parker, and Anne Van der Veen. Agent-based urban land markets: agent's pricing behavior, land prices and urban land use change. Journal of Artificial Societies and Social Simulation, I2(I):3, 2009.

Robert W. Floyd. Algorithm 97: shortest path. Communications of the $A C M, 5(6): 345$, 1962.

Jay W. Forrester. Industrial dynamics. a major breakthrough for decision makers. Harvard business review, 36(4):37-66, 1958 .

Luca Fraccascia and Devrim Murat Yazan. The role of online information-sharing platforms on the performance of industrial symbiosis networks. Resources, Conservation and Recycling, 2018.

Luca Fraccascia, Ilaria Giannoccaro, and Vito Albino. Efficacy of landfill tax and subsidy policies for the emergence of industrial symbiosis networks: An agent-based simulation study. Sustainability, 9(4):521, 2017 a.

Luca Fraccascia, Ilaria Giannoccaro, and Vito Albino. Rethinking resilience in industrial symbiosis: Conceptualization and measurements. Ecological Economics, 137:I48-162, 20I7b. 
Luca Fraccascia, Vahid Yazdanpanah, Guido van Capelleveen, and Devim Murat Yazan. Energy-based industrial symbiosis: A literature review for circular energy transition. Environment, Development and Sustainability, page (under review), 2019.

Luca Fraccascia, Vahid Yazdanpanah, Guido van Capelleveen, and Devrim Murat Yazan. A framework for industrial symbiosis systems for agent-based simulation. In 2019 IEEE 2Ist Conference on Business Informatics (CBI), volume or, pages 419-428, July 2019. doi: Io.II09/CBI.2019.00055.

Iván García-Magariño. A collection of method fragments automated with model transformations in agent-oriented modeling. Engineering Applications of Artificial Intelligence, 26 (3):II3I-II48, 2013.

Iván García-Magariño, Jorge J. Gómez-Sanz, and José R. Pérez-Agüera. A multi-agent based implementation of a delphi process. In Proceedings of the 7 th international joint conference on Autonomous agents and multiagent systems-Volume 3, pages 1543-1546. International Foundation for Autonomous Agents and Multiagent Systems, 2008.

Anna Gatzioura, Miquel Sànchez-Marrè, and Karina Gibert. A hybrid recommender system for industrial symbiotic networks. In Proceedings of gth International Congress on Environmental Modelling and Software (iEMSs-2018), 2018.

Anna Gatzioura, Miquel Sànchez-Marrè, and Karina Gibert. A hybrid recommender system to improve circular economy in industrial symbiotic networks. Energies, I2(I8):3546, 2019.

Yong Geng, Jia Fu, Joseph Sarkis, and Bing Xue. Towards a national circular economy indicator system in china: an evaluation and critical analysis. Journal of cleaner production, 23 (I):216-224, 2012.

Yong Geng, Zuoxi Liu, Bing Xue, Huijuan Dong, Tsuyoshi Fujita, and Anthony Chiu. Emergy-based assessment on industrial symbiosis: a case of shenyang economic and technological development zone. Environmental Science and Pollution Research, 2I(23):13572I3587, 2014 .

Geoffrey Gordon. A general purpose systems simulation program. In Proceedings of the December I2-I4, I96I, eastern joint computer conference: computers-key to total systems control, pages 87-I04. ACM, 196I.

Henning Grann. The industrial symbiosis at kalundborg, denmark. The industrial green game. Implications for environmental design and management, pages I17-123, 1997 . 
Gabriel B. Grant, Thomas P. Seager, Guillaume Massard, and Loring Nies. Information and communication technology for industrial symbiosis. Journal of Industrial Ecology, I4 (5):740-753, 2010 .

Hilary G. Grimes-Casey, Thomas P. Seager, Thomas L. Theis, and Susan E. Powers. A game theory framework for cooperative management of refillable and disposable bottle lifecycles. Journal of Cleaner Production, I5(17):1618-1627, 2007.

Davide Grossi, Luca Tummolini, and Paolo Turrini. Norms in game theory. In Agreement Technologies, pages 19I-197. Springer, 2013.

Edeltraud Guenther and Vera Greschner Farkavcová. Decision making for transportation systems as a support for sustainable stewardship: freight transport process evaluation using the etienne-tool. Management Research Review, 33(4):317-339, 2010.

Mingyu Guo and Vincent Conitzer. Optimal-in-expectation redistribution mechanisms. In Proceedings of the 7 th international joint conference on Autonomous agents and multiagent systems-Volume 2, pages I047-I054. International Foundation for Autonomous Agents and Multiagent Systems, 2008.

David Hamilton. Evolutionary economics: A study of change in economic thought. Routledge, 2017.

Sergiu Hart. Shapley value. In Game Theory, pages 210-216. Springer, 1989.

Manfred J. Holler and Stefan Napel. Monotonicity of power and power measures. Theory and Decision, 56(I-2):93-III, 2004.

Bryan Horling and Victor Lesser. A survey of multi-agent organizational paradigms. The Knowledge engineering review, I9(4):28I-316, 2004.

Trung Dong Huynh, Nicholas R. Jennings, and Nigel R. Shadbolt. An integrated trust and reputation model for open multi-agent systems. JAAMAS, I3(2):I19-154, 2006.

Samuel Ieong and Yoav Shoham. Marginal contribution nets: a compact representation scheme for coalitional games. In Proceedings of the 6th ACM conference on Electronic commerce, pages 193-202. ACM, 2005.

IRIS. Technology Solutions. http://www. iris-eng.com/, 2019. Accessed: 2019-07-0I.

Noel Brings Jacobsen. Industrial symbiosis in kalundborg, denmark: a quantitative assessment of economic and environmental aspects. Journal of industrial ecology, IO(I-2):239-255, 2006. 
Nicholas R. Jennings. Cooperation in industrial multi-agent systems, volume 43. World Scientific, 1994 .

Jan Johanson and Lars-Gunnar Mattsson. Internationalisation in industrial systems-a network approach. In Knowledge, Networks and Power, pages III-I32. Springer, 2015.

Jeffrey Johnson, Andrzej Nowak, Paul Ormerod, Bridget Rosewell, and Yi-Cheng Zhang. Non-Equilibrium Social Science and Policy: Introduction and Essays on New and Changing Paradigms in Socio-Economic Thinking. Springer, 2017.

Thomas M. Jones. Corporate social responsibility revisited, redefined. California management review, 22(3):59-67, 1980.

Souhila Kaci and Leendert van der Torre. Preference-based argumentation: Arguments supporting multiple values. International Journal of Approximate Reasoning, 48(3):73075I, 2008.

Daniel Kahneman. Thinking, fast and slow. Macmillan, 20II.

Ali Kakhbod. Resource allocation in decentralized systems with strategic agents: an implementation theory approach. Springer Science \& Business Media, 2013.

Dimosthenis Kaponis and Jeremy Pitt. Dynamic specifications in norm-governed open computational societies. In International Workshop on Engineering Societies in the Agents World, pages 265-283. Springer, 2006.

Magnus Karlsson and Anna Wolf. Using an optimization model to evaluate the economic benefits of industrial symbiosis in the forest industry. Journal of Cleaner Production, I6(I4): I536-I544, 2008.

Michael Kearns. Graphical games. The New Palgrave Dictionary of Economics: Volume $I-8$, pages $2547-2549,2008$.

Julian Kirchherr, Denise Reike, and Marko Hekkert. Conceptualizing the circular economy: An analysis of II4 definitions. Resources, Conservation and Recycling, I27:22I-232, 2017.

Mark Klein, Juan-Antonio Rodriguez-Aguilar, and Chrysanthos Dellarocas. Using domain-independent exception handling services to enable robust open multi-agent systems: The case of agent death. JAAMAS, 7(I-2):I79-189, 2003.

Rob Kling. Cooperation, coordination and control in computer-supported work. Communications of the $A C M$, 34(I2):83-88, I99I. 
Thomas A. Kochan, Robert B. McKersie, and Peter Cappelli. Strategic choice and industrial relations theory. Industrial Relations: A Journal of Economy and Society, 23(I):16-39, 1984.

David M. Kreps. Game theory and economic modelling. Oxford University Press, 1990.

Kuldeep Kumar and Jos van Hillegersberg. Enterprise resource planning: introduction. Communications of the $A C M, 43(4): 22-26,2000$.

Tian Lan, David T. H. Kao, Mung Chiang, and Ashutosh Sabharwal. An axiomatic theory of fairness in network resource allocation. In INFOCOM 20I0. 29th IEEE International Conference on Computer Communications, pages I343-I35I, 2010.

Jean Lemaire. An application of game theory: cost allocation. Astin Bulletin, I4(or):6I-8I, 1984.

Julien Lesca, Patrice Perny, and Makoto Yokoo. Coalition structure generation and cs-core: Results on the tractability frontier for games represented by mc-nets. In Proceedings of the I6th Conference on Autonomous Agents and MultiAgent Systems, pages 308-316. International Foundation for Autonomous Agents and Multiagent Systems, 2017.

Cuihong Li, Uday Rajan, Shuchi Chawla, and Katia Sycara. Mechanisms for coalition formation and cost sharing in an electronic marketplace. In Proceedings of the sth international conference on Electronic commerce, pages 68-77. ACM, 2003.

Marko Lindroos. Sharing the benefits of cooperation in the norwegian spring-spawning herring fishery. International Game Theory Review, 6(oI):35-53, 2004.

Stephen C. Littlechild and Guillermo Owen. A simple expression for the shapley value in a special case. Management Science, 20(3):370-372, 1973.

D. Rachel Lombardi and Peter Laybourn. Redefining industrial symbiosis. Journal of Industrial Ecology, 16(I):28-37, 2012.

Eva M. Lovelady and Mahmoud M. El-Halwagi. Design and integration of eco-industrial parks for managing water resources. Environmental progress Eீ sustainable energy, 28(2): $265-272,2009$.

Sebastián Lozano, Placido Moreno, Belarmino Adenso-Díaz, and E. Algaba. Cooperative game theory approach to allocating benefits of horizontal cooperation. European Journal of Operational Research, 229(2):444-452, 2013.

Saleh Faraj Magram. Worldwide solid waste recycling strategies: A review. Indian Journal of Science and Technology, 4(6):692-702, 2011. 
Thomas W. Malone. What is coordination theory? National Science Foundation - Coordination Theory Workshop, 1988.

Giorgio Mannina, Taise Ferreira Rebouças, Alida Cosenza, Miquel Sànchez-Marrè, and Karina Gibert. Decision support systems (dss) for wastewater treatment plants-a review of the state of the art. Bioresource technology, page I2I8I4, 2019.

Jason R. Marden and Adam Wierman. Distributed welfare games. Operations Research, 6I (I):I55-I68, 2013 .

Andreu Mas-Colell, Michael Dennis Whinston, Jerry R. Green, et al. Microeconomic theory, volume i. Oxford university press New York, 1995.

Sara Mehryar, Richard Sliuzas, Ali Sharifi, Diana Reckien, and Martin van Maarseveen. A structured participatory method to support policy option analysis in a social-ecological system. Journal of Environmental Management, 197:360-372, 2017.

Sara Mehryar, Richard Sliuzas, Nina Schwarz, Ali Sharifi, and Martin van Maarseveen. From individual fuzzy cognitive maps to agent based models: Modeling multi-factorial and multistakeholder decision-making for water scarcity. Journal of Environmental Management, 250: I09482, 2019 .

Reshef Meir, Jeffrey S. Rosenschein, and Enrico Malizia. Subsidies, stability, and restricted cooperation in coalitional games. In IJCAI 20II, Proceedings of the 22nd International Joint Conference on Artificial Intelligence, Barcelona, Catalonia, Spain, July I6-22, 20II, pages 30I-306, 20II.

Merriam-Webster Online. Merriam-Webster Online Dictionary, 20I9. URL http: //www. merriam-webster.com.

Katharina Michaelowa and Axel Michaelowa. Transnational climate governance initiatives: designed for effective climate change mitigation? International Interactions, 43(I):I29-I55, 2017.

Nicholas R. Miller. Power in game forms. In Power, voting, and voting power, pages 33-5I. Springer, 198I.

Murat Mirata. Experiences from early stages of a national industrial symbiosis programme in the uk: determinants and coordination challenges. Journal of Cleaner Production, I2 (8-IO):967-983, 2004. 
Sanjay Modgil and Henry Prakken. A general account of argumentation with preferences. Artificial Intelligence, 195:36I-397, 2013.

Ludovic Montastruc, Marianne Boix, Luc Pibouleau, Catherine Azzaro-Pantel, and Serge Domenech. On the flexibility of an eco-industrial park (eip) for managing industrial water. Journal of Cleaner Production, 43:I-II, 20I3.

Alan Murray, Keith Skene, and Kathryn Haynes. The circular economy: An interdisciplinary exploration of the concept and application in a global context. Journal of Business Ethics, pages I-I2, 2015.

Roger B. Myerson. Graphs and cooperation in games. Mathematics of operations research, 2(3):225-229, 1977 .

Richard G. Newell. The role of markets and policies in delivering innovation for climate change mitigation. Oxford Review of Economic Policy, 26(2):253-269, 2010.

Noam Nisan, Tim Roughgarden, Eva Tardos, and Vijay V. Vazirani. Algorithmic game theory. Cambridge University Press, 2007.

Martin J. Osborne. An introduction to game theory, volume 3. Oxford university press New York, 2004.

Martin J Osborne and Ariel Rubinstein. A course in game theory. MIT press, 1994.

Sascha Ossowski. Agreement technologies, volume 8. Springer Science \& Business Media, 2012.

Elinor Ostrom. Governing the commons. Cambridge university press, 2015.

Hung-Suck Park and Shishir Kumar Behera. Methodological aspects of applying ecoefficiency indicators to industrial symbiosis networks. Journal of Cleaner Production, 64: $478-485,2014$.

David W. Pearce and R. Kerry Turner. Economics of natural resources and the environment. JHU Press, I990.

Judea Pearl. Theoretical impediments to machine learning with seven sparks from the causal revolution. arXiv preprint arXiv:1801.04016, 2018.

Judea Pearl and Dana Mackenzie. The book of why: the new science of cause and effect. Basic Books, 2018. 
Steve Phelps, Peter McBurney, and Simon Parsons. Evolutionary mechanism design: a review. Autonomous Agents and Multi-Agent Systems, 2I(2):237-264, 2010.

Steven Pinker. Enlightenment now: The case for reason, science, bumanism, and progress. Penguin, 2018.

Isaac Pinyol and Jordi Sabater-Mir. Computational trust and reputation models for open multi-agent systems: a review. Artificial Intelligence Review, 40(I):I-25, 2013.

Peter Poschen. Decent work, green Jobs and the sustainable economy: Solutions for climate change and sustainable development. Routledge, 2017.

John Winsor Pratt and Richard Jay Zeckhauser. The fair and efficient division of the winsor family silver. Management Science, 36(II):1293-I30I, 1990.

Anand S. Rao and Michael P. Georgeff. BDI agents: From theory to practice. In Proceedings of the First International Conference on Multiagent Systems, June I2-I4, 1995, San Francisco, California, USA, pages 312-319, 1995.

Sara Rodriguez, Vicente Julián, Javier Bajo, Carlos Carrascosa, Vicent Botti, and Juan M. Corchado. Agent-based virtual organization architecture. Engineering Applications of Artificial Intelligence, 24(5):895-910, 2011.

Alvin E. Roth. Who Gets What-and Why: The New Economics of Matchmaking and Market Design. Houghton Mifflin Harcourt, 2015.

Alvin E. Roth and Marilda Sotomayor. Two-sided matching. Handbook of game theory with economic applications, I:485-54I, 1992.

Alvin E. Roth and Robert E. Verrecchia. The shapley value as applied to cost allocation: a reinterpretation. Journal of Accounting Research, pages 295-303, 1979.

Alvin E. Roth, Tayfun Sönmez, and M. Utku Ünver. Pairwise kidney exchange. Journal of Economic theory, I25(2):I5I-I88, 2005.

Rossella Rubino, Andrea Omicini, and Enrico Denti. Computational institutions for modelling norm-regulated MAS: An approach based on coordination artifacts. In Proceedings of $A A M A S$-2005, pages 127-I4I. Springer, 2005.

Eusiel Rubio-Castro, José María Ponce-Ortega, Medardo Serna-González, Arturo JiménezGutiérrez, and Mahmoud M El-Halwagi. A global optimal formulation for the water integration in eco-industrial parks considering multiple pollutants. Computers E\& Chemical Engineering, 35(8):1558-1574, 2011. 
John R. Searle. What is an institution? Journal of institutional economics, I(I):I-22, 2005.

Cristina Sendra, Xavier Gabarrell, and Teresa Vicent. Material flow analysis adapted to an industrial area. Journal of Cleaner Production, I5(17):1706-1715, 2007.

Begum Sertyesilisik and Egemen Sertyesilisik. Eco industrial development: As a way of enhancing sustainable development. Journal of Economic Development, Environment and People, 5(I):6, 2016.

Lloyd S. Shapley. A value for n-person games. Contributions to the Theory of Games, 2(28): 307-317, 1953.

Lloyd S. Shapley. On balanced sets and cores. Naval research logistics quarterly, I4(4):453460, 1967.

Lloyd S. Shapley. Cores of convex games. International journal of game theory, $\mathrm{I}(\mathrm{I}): \mathrm{II}-26$, I97I.

Lloyd S. Shapley and Martin Shubik. On market games. Journal of Economic Theory, I(I): 9-25, 1969.

SHAREBOX. Secure Sharing. http://sharebox-project.eu/, 2019. Accessed: 2019-IIoI.

Mark Sharfman, Rex T. Ellington, and Mark Meo. The next step in becoming "green”: life-cycle oriented environmental management. Business Horizons, 40(3):13-22, 1997.

Yoav Shoham and Moshe Tennenholtz. On social laws for artificial agent societies: off-line design. Artificial intelligence, 73(I-2):23I-252, 1995 .

Riyaz Sikora and Michael J. Shaw. A multi-agent framework for the coordination and integration of information systems. Management Science, 44(II-part-2):S65-S78, 1998.

Brian S. Silverman. Technological resources and the direction of corporate diversification: Toward an integration of the resource-based view and transaction cost economics. Management science, 45(8):1109-II24, 1999 .

Knud Sinding. Environmental management beyond the boundaries of the firm: definitions and constraints. Business strategy and the environment, 9(2):79, 2000.

Munindar P. Singh. Norms as a basis for governing sociotechnical systems. ACM Transactions on Intelligent Systems and Technology (TIST), 5(I):21, 2013. 
Kullapa Soratana and Amy E. Landis. Evaluating industrial symbiosis and algae cultivation from a life cycle perspective. Bioresource technology, IO2(13):6892-690I, $20 \mathrm{II}$.

Craig Standing, Paul Jackson, Joseph Sarkis, and Hanmin Zhu. Information technology and systems in china's circular economy: Implications for sustainability. Journal of Systems and Information Technology, IO(3):202-217, 2008.

Lu Sun, Hong Li, Liang Dong, Kai Fang, Jingzheng Ren, Yong Geng, Minoru Fujii, Wei Zhang, Ning Zhang, and Zhe Liu. Eco-benefits assessment on urban industrial symbiosis based on material flows analysis and emergy evaluation approach: A case of liuzhou city, china. Resources, Conservation and Recycling, 119:78-88, 2017.

Pankaj R. Telang and Munindar P. Singh. Specifying and verifying cross-organizational business models: An agent-oriented approach. IEEE Transactions on Services Computing, $5(3): 305-318,2012$.

Richard H. Thaler. Behavioral economics: Past, present, and future. American Economic Review, 106(7):1577-1600, 2016.

William Thomson. The fair division of a fixed supply among a growing population. Mathematics of Operations Research, 8(3):319-326, 1983.

Nick Tinnemeier, Mehdi Dastani, and John-Jules Meyer. Programming norm change. In Proceedings of AAMAS 2010, pages 957-964, 2010.

Rene Van Berkel, Tsuyoshi Fujita, Shizuka Hashimoto, and Yong Geng. Industrial and urban symbiosis in japan: Analysis of the eco-town program 1997-2006. Journal of Environmental Management, 90(3):1544-1556, 2009.

Guido van Capelleveen, Chintan Amrit, and Devrim Murat Yazan. A literature survey of information systems facilitating the identification of industrial symbiosis. In From Science to Society, pages 155-169. Springer, 2018.

Egon L. Van den Broek, Catholijn M. Jonker, Alexei Sharpanskykh, Jan Treur, et al. Formal modeling and analysis of organizations. In International Conference on Autonomous Agents and Multiagent Systems, pages 18-34. Springer, 2005.

Wiebe Van der Hoek and Michael Wooldridge. Cooperation, knowledge, and time: Alternating-time temporal epistemic logic and its applications. Studia logica, 75(I):125-157, 2003. 
Jos van Hillegersberg, Hans Moonen, and Simon Dalmolen. Coordination as a service to enable agile business networks. In International Workshop on Global Sourcing of Information Technology and Business Processes, pages 164-174, 2012.

Nir Vulkan and Nicholas R. Jennings. Efficient mechanisms for the supply of services in multi-agent environments. Decision Support Systems, 28(I-2):5-19, 2000.

Yonghong Wang and Munindar P. Singh. Formal trust model for multiagent systems. In IJCAI, volume 7, pages I55I-I556, 2007.

Yorai Wardi, Christos G. Cassandras, and Xi-Ren Cao. Perturbation analysis: A framework for data-driven control and optimization of discrete event and hybrid systems. Annual Reviews in Control, 45:267-280, 2018. doi: 10.1016/j.arcontrol.2018.04.003. URL https: //doi.org/10.1016/j.arcontrol.2018.04.003.

Bo Weidema. Avoiding co-product allocation in life-cycle assessment. Journal of industrial ecology, 4(3):II-33, 2000.

Oliver E. Williamson. Transaction-cost economics: the governance of contractual relations. The journal of Law and Economics, 22(2):233-26I, 1979.

Oliver E. Williamson. The economics of organization: The transaction cost approach. American journal of sociology, pages 548-577, 198I.

Oliver E. Williamson. The economic institutions of capitalism: firms, markets, relational contracting. Free Press, 1985.

Oliver E. Williamson. Transaction cost economics and organization theory. Industrial and corporate change, 2(2):107-156, 1993.

Anna Wolf, Mats Eklund, and Mats Söderström. Developing integration in a local industrial ecosystem-an explorative approach. Business Strategy and the Environment, 16(6):442-455, 2007 .

Michael Wooldridge. An introduction to multiagent systems. John Wiley \& Sons, 2009.

Michael Wooldridge and Nicholas R. Jennings. Intelligent agents: Theory and practice. The knowledge engineering review, $10(2): 115-152,1995$.

Michael Wooldridge, Ulle Endriss, Sarit Kraus, and Jérôme Lang. Incentive engineering for boolean games. Artificial Intelligence, 195:418-439, 2013. 
Zofia Wysokińska. The "new" environmental policy of the european union: A path to development of a circular economy and mitigation of the negative effects of climate change. Comparative Economic Research, 19(2):57-73, 2016.

Devrim Murat Yazan. Constructing joint production chains: An enterprise input-output approach for alternative energy use. Resources, conservation and recycling, 107:38-52, 2016.

Devrim Murat Yazan, Joy Clancy, and Jon Lovett. Supply chains, techno-economic assessment and market development for second generation biodiesel. Advances in biodiesel production: processes and technologies, pages 254-280, 2012.

Devrim Murat Yazan, Vincenzo Alessio Romano, and Vito Albino. The design of industrial symbiosis: an input-output approach. Journal of Cleaner Production, 129:537-547, 2016.

Devrim Murat Yazan, Vahid Yazdanpanah, and Luca Fraccascia. Cooperation decisions in industrial symbiotic relations. In 25th International Input-Output Conference (IIOA 20I7), 2017a.

Devrim Murat Yazan, Vahid Yazdanpanah, and Luca Fraccascia. Learning fair play in industrial symbiotic relations. In Proceedings of the First Symbiosis User Network Conference on Methods and Tools for the Implementation of Industrial Symbiosis, pages 49-50, 2017b.

Devrim Murat Yazan, Vahid Yazdanpanah, and Luca Fraccascia. Strategic cooperative behavior in industrial symbiosis: A game-theoretical approach integrated with agent-based simulation. Business Strategy and the Environment, page (under review), 2019.

Vahid Yazdanpanah and Devrim Murat Yazan. Industrial symbiotic relations as cooperative games. In Proceedings of the 7 th International Conference on Industrial Engineering and Systems Management (IESM-20I7), pages 455-460, 2017.

Vahid Yazdanpanah, Devrim Murat Yazan, and W. Henk M. Zijm. Normative industrial symbiotic networks: A position paper. In Multi-Agent Systems and Agreement Technologies - I4th European Conference, EUMAS 2016, and 4th International Conference, AT 2016, Valencia, Spain, December I5-16, 2016, Revised Selected Papers, pages 314-321, 2016.

Vahid Yazdanpanah, Devrim Murat Yazan, and W. Henk M. Zijm. Industrial symbiotic networks as coordinated games. In Proceedings of the I7th International Conference on Autonomous Agents and MultiAgent Systems, AAMAS 2018, Stockholm, Sweden, July 1o-I5, 2018, pages 2145-2147, 2018. 
Vahid Yazdanpanah, Devrim Murat Yazan, Jos van Hillegersberg, and Mehdi Dastani. An introduction to engineering multiagent industrial symbiosis systems: Potentials and challenges. Presented at 7 th International Workshop on Engineering Multi-Agent Systems (EMAS 2019), Montreal, Canada, I3th-I4th May, 2orga.

Vahid Yazdanpanah, Devrim Murat Yazan, and W. Henk M. Zijm. Self-governing industrial symbiosis. (Working Paper), 2orgb.

Vahid Yazdanpanah, Devrim Murat Yazan, and W. Henk M. Zijm. Coordinated multiagent industrial symbiosis systems. Automatica, pages (under-review), 2019c.

Vahid Yazdanpanah, Devrim Murat Yazan, and W. Henk M. Zijm. Dynamics and allocation of transaction cost in multiagent industrial symbiosis. Computers E\& Industrial Engineering, page (submitted), 2orgd.

Vahid Yazdanpanah, Devrim Murat Yazan, and W. Henk M. Zijm. FISOF: A formal industrial symbiosis opportunity filtering method. Engineering Applications of Artificial Intelligence, 81:247-259, 2019e.

H. Peyton Young. Cost allocation: methods, principles, applications. North Holland Publishing Co., I985a.

$\mathrm{H}$ Peyton Young. Monotonic solutions of cooperative games. International Journal of Game Theory, I4(2):65-72, 1985b.

Zengwei Yuan, Jun Bi, and Yuichi Moriguichi. The circular economy: A new development strategy in china. Journal of Industrial Ecology, IO(I-2):4-8, 2006.

Bin Zhang, Zhaohua Wang, and Kee-hung Lai. Does industrial waste reuse bring dual benefits of economic growth and carbon emission reduction?: Evidence of incorporating the indirect effect of economic growth in china. Journal of Industrial Ecology, 2015.

Qinghua Zhu, Ernest A. Lowe, Yuan-an Wei, and Donald Barnes. Industrial symbiosis in china: a case study of the guitang group. Journal of Industrial Ecology, II(I):3I-42, 2007.

Yair Zick, Maria Polukarov, and Nicholas R. Jennings. Taxation and stability in cooperative games. In International conference on Autonomous Agents and Multi-Agent Systems, AAMAS 'I3, Saint Paul, MN, USA, May 6-10, 2013, pages 523-530, 2013.

W. Henk M. Zijm, Matthias Klumpp, Uwe Clausen, and Michael Ten Hompel. Logistics and Supply Chain Innovation: Bridging the Gap between Theory and Practice. Springer, 2015. 


\section{SIKS dissertations published since 2OII}

$2 \mathrm{OII}$

oI Botond Cseke (RUN), Variational Algorithms for Bayesian Inference in Latent Gaussian Models

O2 Nick Tinnemeier (UU), Organizing Agent Organizations. Syntax and Operational Semantics of an Organization-Oriented Programming Language

o3 Jan Martijn van der Werf(TUE), Compositional Design and Verification of ComponentBased Information Systems

04 Hado van Hasselt (UU), Insights in Reinforcement Learning; Formal analysis and empirical evaluation of temporal-difference

os Bas van der Raadt (VU), Enterprise Architecture Coming of Age - Increasing the Performance of an Emerging Discipline.

o6 Yiwen Wang (TUE), Semantically-Enhanced Recommendations in Cultural Heritage

o7 Yujia Cao (UT), Multimodal Information Presentation for High Load Human Computer Interaction

o8 Nieske Vergunst (UU), BDI-based Generation of Robust Task-Oriented Dialogues

o9 Tim de Jong (OU), Contextualised Mobile Media for Learning

Io Bart Bogaert (UvT), Cloud Content Contention

II Dhaval Vyas (UT), Designing for Awareness: An Experience-focused HCI Perspective

I2 Carmen Bratosin (TUE), Grid Architecture for Distributed Process Mining

I3 Xiaoyu Mao (UvT), Airport under Control. Multiagent Scheduling for Airport Ground Handling

I4 Milan Lovric (EUR), Behavioral Finance and Agent-Based Artificial Markets

I5 Marijn Koolen (UvA), The Meaning of Structure: the Value of Link Evidence for Information Retrieval

I6 Maarten Schadd (UM), Selective Search in Games of Different Complexity 
I7 Jiyin He (UVA), Exploring Topic Structure: Coherence, Diversity and Relatedness

I8 Mark Ponsen (UM), Strategic Decision-Making in complex games

I9 Ellen Rusman (OU), The Mind's Eye on Personal Profiles

20 Qing Gu (VU), Guiding service-oriented software engineering - A view-based approach

2I Linda Terlouw (TUD), Modularization and Specification of Service-Oriented Systems

22 Junte Zhang (UVA), System Evaluation of Archival Description and Access

23 Wouter Weerkamp (UVA), Finding People and their Utterances in Social Media

24 Herwin van Welbergen (UT), Behavior Generation for Interpersonal Coordination with Virtual Humans On Specifying, Scheduling and Realizing Multimodal Virtual Human Behavior

25 Syed Waqar ul Qounain Jaffry (VU), Analysis and Validation of Models for Trust Dynamics

26 Matthijs Aart Pontier (VU), Virtual Agents for Human Communication - Emotion Regulation and Involvement-Distance Trade-Offs in Embodied Conversational Agents and Robots

27 Aniel Bhulai (VU), Dynamic website optimization through autonomous management of design patterns

28 Rianne Kaptein (UVA), Effective Focused Retrieval by Exploiting Query Context and Document Structure

29 Faisal Kamiran (TUE), Discrimination-aware Classification

30 Egon van den Broek (UT), Affective Signal Processing (ASP): Unraveling the mystery of emotions

3I Ludo Waltman (EUR), Computational and Game-Theoretic Approaches for Modeling Bounded Rationality

32 Nees-Jan van Eck (EUR), Methodological Advances in Bibliometric Mapping of Science

33 Tom van der Weide (UU), Arguing to Motivate Decisions

34 Paolo Turrini (UU), Strategic Reasoning in Interdependence: Logical and Gametheoretical Investigations

35 Maaike Harbers (UU), Explaining Agent Behavior in Virtual Training

36 Erik van der Spek (UU), Experiments in serious game design: a cognitive approach

37 Adriana Burlutiu (RUN), Machine Learning for Pairwise Data, Applications for Preference Learning and Supervised Network Inference

38 Nyree Lemmens (UM), Bee-inspired Distributed Optimization 
39 Joost Westra (UU), Organizing Adaptation using Agents in Serious Games

40 Viktor Clerc (VU), Architectural Knowledge Management in Global Software Development

4I Luan Ibraimi (UT), Cryptographically Enforced Distributed Data Access Control

42 Michal Sindlar(UU), Explaining Behavior through Mental State Attribution

43 Henk van der Schuur (UU), Process Improvement through Software Operation Knowledge

44 Boris Reuderink(UT), Robust Brain-Computer Interfaces

45 Herman Stehouwer (UvT), Statistical Language Models for Alternative Sequence Selection

46 Beibei Hu (TUD), Towards Contextualized Information Delivery: A Rule-based Architecture for the Domain of Mobile Police Work

47 Azizi Bin Ab Aziz (VU), Exploring Computational Models for Intelligent Support of Persons with Depression

48 Mark Ter Maat (UT), Response Selection and Turn-taking for a Sensitive Artificial Listening Agent

49 Andreea Niculescu (UT), Conversational interfaces for task-oriented spoken dialogues: design aspects influencing interaction quality

2012

or Terry Kakeeto (UvT), Relationship Marketing for SMEs in Uganda

O2 Muhammad Umair (VU), Adaptivity, emotion, and Rationality in Human and Ambient Agent Models

03 Adam Vanya (VU), Supporting Architecture Evolution by Mining Software Repositories

o4 Jurriaan Souer (UU), Development of Content Management System-based Web Applications

os Marijn Plomp (UU), Maturing Interorganisational Information Systems

o6 Wolfgang Reinhardt (OU), Awareness Support for Knowledge Workers in Research Networks

o7 Rianne van Lambalgen (VU), When the Going Gets Tough: Exploring Agent-based Models of Human Performance under Demanding Conditions

o8 Gerben de Vries (UVA), Kernel Methods for Vessel Trajectories

o9 Ricardo Neisse (UT), Trust and Privacy Management Support for Context-Aware Service Platforms

Io David Smits (TUE), Towards a Generic Distributed Adaptive Hypermedia Environment 
II J.C.B. Rantham Prabhakara (TUE), Process Mining in the Large: Preprocessing, Discovery, and Diagnostics

I2 Kees van der Sluijs (TUE), Model Driven Design and Data Integration in Semantic Web Information Systems

I3 Suleman Shahid (UvT), Fun and Face: Exploring non-verbal expressions of emotion during playful interactions

I4 Evgeny Knutov (TUE), Generic Adaptation Framework for Unifying Adaptive Web-based Systems

is Natalie van der Wal (VU), Social Agents. Agent-Based Modelling of Integrated Internal and Social Dynamics of Cognitive and Affective Processes.

I6 Fiemke Both (VU), Helping people by understanding them - Ambient Agents supporting task execution and depression treatment

17 Amal Elgammal (UvT), Towards a Comprehensive Framework for Business Process Compliance

I8 Eltjo Poort (VU), Improving Solution Architecting Practices

19 Helen Schonenberg (TUE), What's Next? Operational Support for Business Process Execution

20 Ali Bahramisharif(RUN), Covert Visual Spatial Attention, a Robust Paradigm for BrainComputer Interfacing

2I Roberto Cornacchia (TUD), Querying Sparse Matrices for Information Retrieval

22 Thijs Vis (UvT), Intelligence, politie en veiligheidsdienst: verenigbare grootheden?

23 Christian Muehl (UT), Toward Affective Brain-Computer Interfaces: Exploring the Neurophysiology of Affect during Human Media Interaction

24 Laurens van der Werff (UT), Evaluation of Noisy Transcripts for Spoken Document Retrieval

25 Silja Eckartz (UT), Managing the Business Case Development in Inter-Organizational IT Projects: A Methodology and its Application

26 Emile de Maat (UVA), Making Sense of Legal Text

27 Hayrettin Gurkok(UT), Mind the Sheep! User Experience Evaluation \& Brain-Computer Interface Games

28 Nancy Pascall (UvT), Engendering Technology Empowering Women

29 Almer Tigelaar (UT), Peer-to-Peer Information Retrieval

30 Alina Pommeranz (TUD), Designing Human-Centered Systems for Reflective Decision Making 
31 Emily Bagarukayo (RUN), A Learning by Construction Approach for Higher Order Cognitive Skills Improvement, Building Capacity and Infrastructure

32 Wietske Visser(TUD), Qualitative multi-criteria preference representation and reasoning

33 Rory Sie (OUN), Coalitions in Cooperation Networks (COCOON)

34 Pavol Jancura (RUN), Evolutionary analysis in PPI networks and applications

35 Evert Haasdijk (VU), Never Too Old To Learn - On-line Evolution of Controllers in Swarm- and Modular Robotics

36 Denis Ssebugwawo (RUN), Analysis and Evaluation of Collaborative Modeling Processes

37 Agnes Nakakawa (RUN), A Collaboration Process for Enterprise Architecture Creation

38 Selmar Smit (VU), Parameter Tuning and Scientific Testing in Evolutionary Algorithms

39 Hassan Fatemi (UT), Risk-aware design of value and coordination networks

40 Agus Gunawan (UvT), Information Access for SMEs in Indonesia

4I Sebastian Kelle (OU), Game Design Patterns for Learning

42 Dominique Verpoorten (OU), Reflection Amplifiers in self-regulated Learning

43 Anna Tordai (VU), On Combining Alignment Techniques

44 Benedikt Kratz (UvT), A Model and Language for Business-aware Transactions

45 Simon Carter(UVA), Exploration and Exploitation of Multilingual Data for Statistical Machine Translation

46 Manos Tsagkias (UVA), Mining Social Media: Tracking Content and Predicting Behavior

47 Jorn Bakker (TUE), Handling Abrupt Changes in Evolving Time-series Data

48 Michael Kaisers (UM), Learning against Learning - Evolutionary dynamics of reinforcement learning algorithms in strategic interactions

59 Steven van Kervel (TUD), Ontologogy driven Enterprise Information Systems Engineering

so Jeroen de Jong (TUD), Heuristics in Dynamic Sceduling; a practical framework with a case study in elevator dispatching

2013

or Viorel Milea (EUR), News Analytics for Financial Decision Support

O2 Erietta Liarou (CWI), MonetDB/DataCell: Leveraging the Column-store Database Technology for Efficient and Scalable Stream Processing

o3 Szymon Klarman (VU), Reasoning with Contexts in Description Logics 
o4 Chetan Yadati (TUD), Coordinating autonomous planning and scheduling

os Dulce Pumareja (UT), Groupware Requirements Evolutions Patterns

o6 Romulo Goncalves (CWI), The Data Cyclotron: Juggling Data and Queries for a Data Warehouse Audience

07 Giel van Lankveld (UvT), Quantifying Individual Player Differences

o8 Robbert-Jan Merk (VU), Making enemies: cognitive modeling for opponent agents in fighter pilot simulators

o9 Fabio Gori (RUN), Metagenomic Data Analysis: Computational Methods and Applications

Io Jeewanie Jayasinghe Arachchige (UvT), A Unified Modeling Framework for Service Design.

II Evangelos Pournaras (TUD), Multi-level Reconfigurable Self-organization in Overlay Services

I2 Marian Razavian (VU), Knowledge-driven Migration to Services

I3 Mohammad Safiri (UT), Service Tailoring: User-centric creation of integrated IT-based homecare services to support independent living of elderly

I4 Jafar Tanha (UVA), Ensemble Approaches to Semi-Supervised Learning Learning

I5 Daniel Hennes (UM), Multiagent Learning - Dynamic Games and Applications

I6 Eric Kok (UU), Exploring the practical benefits of argumentation in multi-agent deliberation

17 Koen Kok (VU), The PowerMatcher: Smart Coordination for the Smart Electricity Grid

I8 Jeroen Janssens (UvT), Outlier Selection and One-Class Classification

I9 Renze Steenhuizen (TUD), Coordinated Multi-Agent Planning and Scheduling

20 Katja Hofmann (UvA), Fast and Reliable Online Learning to Rank for Information Retrieval

2I Sander Wubben (UvT), Text-to-text generation by monolingual machine translation

22 Tom Claassen (RUN), Causal Discovery and Logic

23 Patricio de Alencar Silva (UvT), Value Activity Monitoring

24 Haitham Bou Ammar (UM), Automated Transfer in Reinforcement Learning

25 Agnieszka Anna Latoszek-Berendsen (UM), Intention-based Decision Support. A new way of representing and implementing clinical guidelines in a Decision Support System

26 Alireza Zarghami (UT), Architectural Support for Dynamic Homecare Service Provisioning 
27 Mohammad Huq (UT), Inference-based Framework Managing Data Provenance

28 Frans van der Sluis (UT), When Complexity becomes Interesting: An Inquiry into the Information eXperience

29 Iwan de Kok (UT), Listening Heads

30 Joyce Nakatumba (TUE), Resource-Aware Business Process Management: Analysis and Support

3I Dinh Khoa Nguyen (UvT), Blueprint Model and Language for Engineering Cloud Applications

32 Kamakshi Rajagopal (OUN), Networking For Learning; The role of Networking in a Lifelong Learner's Professional Development

33 Qi Gao (TUD), User Modeling and Personalization in the Microblogging Sphere

34 Kien Tjin-Kam-Jet (UT), Distributed Deep Web Search

35 Abdallah El Ali (UvA), Minimal Mobile Human Computer Interaction

36 Than Lam Hoang (TUe), Pattern Mining in Data Streams

37 Dirk Börner (OUN), Ambient Learning Displays

38 Eelco den Heijer (VU), Autonomous Evolutionary Art

39 Joop de Jong (TUD), A Method for Enterprise Ontology based Design of Enterprise Information Systems

40 Pim Nijssen (UM), Monte-Carlo Tree Search for Multi-Player Games

4I Jochem Liem (UVA), Supporting the Conceptual Modelling of Dynamic Systems: A Knowledge Engineering Perspective on Qualitative Reasoning

42 Léon Planken (TUD), Algorithms for Simple Temporal Reasoning

43 Marc Bron (UVA), Exploration and Contextualization through Interaction and Concepts

or Nicola Barile (UU), Studies in Learning Monotone Models from Data

o2 Fiona Tuliyano (RUN), Combining System Dynamics with a Domain Modeling Method

03 Sergio Raul Duarte Torres (UT), Information Retrieval for Children: Search Behavior and Solutions

04 Hanna Jochmann-Mannak (UT), Websites for children: search strategies and interface design - Three studies on children's search performance and evaluation

os Jurriaan van Reijsen (UU), Knowledge Perspectives on Advancing Dynamic Capability

o6 Damian Tamburri (VU), Supporting Networked Software Development 
o7 Arya Adriansyah (TUE), Aligning Observed and Modeled Behavior

o8 Samur Araujo (TUD), Data Integration over Distributed and Heterogeneous Data Endpoints

o9 Philip Jackson (UvT), Toward Human-Level Artificial Intelligence: Representation and Computation of Meaning in Natural Language

Io Ivan Salvador Razo Zapata (VU), Service Value Networks

II Janneke van der Zwaan (TUD), An Empathic Virtual Buddy for Social Support

I2 Willem van Willigen (VU), Look Ma, No Hands: Aspects of Autonomous Vehicle Control

I3 Arlette van Wissen (VU), Agent-Based Support for Behavior Change: Models and Applications in Health and Safety Domains

I4 Yangyang Shi (TUD), Language Models With Meta-information

I5 Natalya Mogles (VU), Agent-Based Analysis and Support of Human Functioning in Complex Socio-Technical Systems: Applications in Safety and Healthcare

i6 Krystyna Milian (VU), Supporting trial recruitment and design by automatically interpreting eligibility criteria

I7 Kathrin Dentler (VU), Computing healthcare quality indicators automatically: Secondary Use of Patient Data and Semantic Interoperability

I8 Mattijs Ghijsen (UVA), Methods and Models for the Design and Study of Dynamic Agent Organizations

I9 Vinicius Ramos (TUE), Adaptive Hypermedia Courses: Qualitative and Quantitative Evaluation and Tool Support

20 Mena Habib (UT), Named Entity Extraction and Disambiguation for Informal Text: The Missing Link

2I Kassidy Clark (TUD), Negotiation and Monitoring in Open Environments

22 Marieke Peeters (UU), Personalized Educational Games - Developing agent-supported scenario-based training

23 Eleftherios Sidirourgos (UvA/CWI), Space Efficient Indexes for the Big Data Era

24 Davide Ceolin (VU), Trusting Semi-structured Web Data

25 Martijn Lappenschaar (RUN), New network models for the analysis of disease interaction

26 Tim Baarslag (TUD), What to Bid and When to Stop

27 Rui Jorge Almeida (EUR), Conditional Density Models Integrating Fuzzy and Probabilistic Representations of Uncertainty

28 Anna Chmielowiec (VU), Decentralized k-Clique Matching 
29 Jaap Kabbedijk (UU), Variability in Multi-Tenant Enterprise Software

30 Peter de Cock (UvT), Anticipating Criminal Behaviour

3I Leo van Moergestel (UU), Agent Technology in Agile Multiparallel Manufacturing and Product Support

32 Naser Ayat (UvA), On Entity Resolution in Probabilistic Data

33 Tesfa Tegegne (RUN), Service Discovery in eHealth

34 Christina Manteli (VU), The Effect of Governance in Global Software Development: Analyzing Transactive Memory Systems.

35 Joost van Ooijen (UU), Cognitive Agents in Virtual Worlds: A Middleware Design Approach

36 Joos Buijs (TUE), Flexible Evolutionary Algorithms for Mining Structured Process Models

37 Maral Dadvar (UT), Experts and Machines United Against Cyberbullying

38 Danny Plass-Oude Bos (UT), Making brain-computer interfaces better: improving usability through post-processing.

39 Jasmina Maric (UvT), Web Communities, Immigration, and Social Capital

40 Walter Omona (RUN), A Framework for Knowledge Management Using ICT in Higher Education

4I Frederic Hogenboom (EUR), Automated Detection of Financial Events in News Text

42 Carsten Eijckhof (CWI/TUD), Contextual Multidimensional Relevance Models

43 Kevin Vlaanderen (UU), Supporting Process Improvement using Method Increments

44 Paulien Meesters (UvT), Intelligent Blauw. Met als ondertitel: Intelligence-gestuurde politiezorg in gebiedsgebonden eenheden.

45 Birgit Schmitz (OUN), Mobile Games for Learning: A Pattern-Based Approach

46 Ke Tao (TUD), Social Web Data Analytics: Relevance, Redundancy, Diversity

47 Shangsong Liang (UVA), Fusion and Diversification in Information Retrieval

2015

or Niels Netten (UvA), Machine Learning for Relevance of Information in Crisis Response

o2 Faiza Bukhsh (Uv'T), Smart auditing: Innovative Compliance Checking in Customs Controls

03 Twan van Laarhoven (RUN), Machine learning for network data

o4 Howard Spoelstra (OUN), Collaborations in Open Learning Environments 
os Christoph Bösch (UT), Cryptographically Enforced Search Pattern Hiding

o6 Farideh Heidari (TUD), Business Process Quality Computation - Computing NonFunctional Requirements to Improve Business Processes

o7 Maria-Hendrike Peetz (UvA), Time-Aware Online Reputation Analysis

o8 Jie Jiang (TUD), Organizational Compliance: An agent-based model for designing and evaluating organizational interactions

o9 Randy Klaassen (UT), HCI Perspectives on Behavior Change Support Systems

io Henry Hermans (OUN), OpenU: design of an integrated system to support lifelong learning

II Yongming Luo(TUE), Designing algorithms for big graph datasets: A study of computing bisimulation and joins

I2 Julie M. Birkholz (VU), Modi Operandi of Social Network Dynamics: The Effect of Context on Scientific Collaboration Networks

I3 Giuseppe Procaccianti (VU), Energy-Efficient Software

I4 Bart van Straalen (UT), A cognitive approach to modeling bad news conversations

I5 Klaas Andries de Graaf(VU), Ontology-based Software Architecture Documentation

I6 Changyun Wei (UT), Cognitive Coordination for Cooperative Multi-Robot Teamwork

17 André van Cleeff(UT), Physical and Digital Security Mechanisms: Properties, Combinations and Trade-offs

I8 Holger Pirk (CWI), Waste Not, Want Not! - Managing Relational Data in Asymmetric Memories

I9 Bernardo Tabuenca (OUN), Ubiquitous Technology for Lifelong Learners

20 Lois Vanhée (UU), Using Culture and Values to Support Flexible Coordination

2I Sibren Fetter (OUN), Using Peer-Support to Expand and Stabilize Online Learning

22 Zhemin Zhu (UT), Co-occurrence Rate Networks

23 Luit Gazendam (VU), Cataloguer Support in Cultural Heritage

24 Richard Berendsen (UVA), Finding People, Papers, and Posts: Vertical Search Algorithms and Evaluation

25 Steven Woudenberg (UU), Bayesian Tools for Early Disease Detection

26 Alexander Hogenboom (EUR), Sentiment Analysis of Text Guided by Semantics and Structure

27 Sándor Héman (CWI), Updating compressed colomn stores 
28 Janet Bagorogoza (TiU), Knowledge Management and High Performance; The Uganda Financial Institutions Model for HPO

29 Hendrik Baier (UM), Monte-Carlo Tree Search Enhancements for One-Player and TwoPlayer Domains

30 Kiavash Bahreini (OU), Real-time Multimodal Emotion Recognition in E-Learning

3I Yakup Koç (TUD), On the robustness of Power Grids

32 Jerome Gard (UL), Corporate Venture Management in SMEs

33 Frederik Schadd (TUD), Ontology Mapping with Auxiliary Resources

34 Victor de Graaf(UT), Gesocial Recommender Systems

35 Jungxao Xu (TUD), Affective Body Language of Humanoid Robots: Perception and Effects in Human Robot Interaction

2016

or Syed Saiden Abbas (RUN), Recognition of Shapes by Humans and Machines

o2 Michiel Christiaan Meulendijk (UU), Optimizing medication reviews through decision support: prescribing a better pill to swallow

o3 Maya Sappelli (RUN), Knowledge Work in Context: User Centered Knowledge Worker Support

o4 Laurens Rietveld (VU), Publishing and Consuming Linked Data

os Evgeny Sherkhonov (UVA), Expanded Acyclic Queries: Containment and an Application in Explaining Missing Answers

o6 Michel Wilson (TUD), Robust scheduling in an uncertain environment

o7 Jeroen de Man (VU), Measuring and modeling negative emotions for virtual training

o8 Matje van de Camp (TiU), A Link to the Past: Constructing Historical Social Networks from Unstructured Data

o9 Archana Nottamkandath (VU), Trusting Crowdsourced Information on Cultural Artefacts

Io George Karafotias (VUA), Parameter Control for Evolutionary Algorithms

II Anne Schuth (UVA), Search Engines that Learn from Their Users

I2 Max Knobbout (UU), Logics for Modelling and Verifying Normative Multi-Agent Systems

I3 Nana Baah Gyan (VU), The Web, Speech Technologies and Rural Development in West Africa - An ICT 4 D Approach

I4 Ravi Khadka (UU), Revisiting Legacy Software System Modernization 
I5 Steffen Michels (RUN), Hybrid Probabilistic Logics - Theoretical Aspects, Algorithms and Experiments

I6 Guangliang Li (UVA), Socially Intelligent Autonomous Agents that Learn from Human Reward

17 Berend Weel (VU), Towards Embodied Evolution of Robot Organisms

I8 Albert Meroño Peñuela (VU), Refining Statistical Data on the Web

I9 Julia Efremova ( Tu/e), Mining Social Structures from Genealogical Data

20 Daan Odijk (UVA), Context \& Semantics in News \& Web Search

2I Alejandro Moreno Célleri (UT), From Traditional to Interactive Playspaces: Automatic Analysis of Player Behavior in the Interactive Tag Playground

22 Grace Lewis (VU), Software Architecture Strategies for Cyber-Foraging Systems

23 Fei Cai (UVA), Query Auto Completion in Information Retrieval

24 Brend Wanders (UT), Repurposing and Probabilistic Integration of Data; An Iterative and data model independent approach

25 Julia Kiseleva (TU/e), Using Contextual Information to Understand Searching and Browsing Behavior

26 Dilhan Thilakarathne (VU), In or Out of Control: Exploring Computational Models to Study the Role of Human Awareness and Control in Behavioural Choices, with Applications in Aviation and Energy Management Domains

27 Wen Li (TUD), Understanding Geo-spatial Information on Social Media

28 Mingxin Zhang (TUD), Large-scale Agent-based Social Simulation - A study on epidemic prediction and control

29 Nicolas Höning (TUD), Peak reduction in decentralised electricity systems - Markets and prices for flexible planning

30 Ruud Mattheij (UvT), The Eyes Have It

3I Mohammad Khelghati (UT), Deep web content monitoring

32 Eelco Vriezekolk (UT), Assessing Telecommunication Service Availability Risks for Crisis Organisations

33 Peter Bloem (UVA), Single Sample Statistics, exercises in learning from just one example

34 Dennis Schunselaar (TUE), Configurable Process Trees: Elicitation, Analysis, and Enactment

35 Zhaochun Ren(UVA), Monitoring Social Media: Summarization, Classification and Recommendation 
36 Daphne Karreman (UT), Beyond $\mathrm{R}_{2} \mathrm{D}_{2}$ : The design of nonverbal interaction behavior optimized for robot-specific morphologies

37 Giovanni Sileno (UvA), Aligning Law and Action - a conceptual and computational inquiry

38 Andrea Minuto (UT), Materials that Matter - Smart Materials meet Art \& Interaction Design

39 Merijn Bruijnes (UT), Believable Suspect Agents; Response and Interpersonal Style Selection for an Artificial Suspect

40 Christian Detweiler (TUD), Accounting for Values in Design

4I Thomas King (TUD), Governing Governance: A Formal Framework for Analysing Institutional Design and Enactment Governance

42 Spyros Martzoukos (UVA), Combinatorial and Compositional Aspects of Bilingual Aligned Corpora

43 Saskia Koldijk(RUN), Context-Aware Support for Stress Self-Management: From Theory to Practice

44 Thibault Sellam (UVA), Automatic Assistants for Database Exploration

45 Bram van de Laar (UT), Experiencing Brain-Computer Interface Control

46 Jorge Gallego Perez (UT), Robots to Make you Happy

47 Christina Weber (UL), Real-time foresight - Preparedness for dynamic innovation networks

48 Tanja Buttler (TUD), Collecting Lessons Learned

49 Gleb Polevoy (TUD), Participation and Interaction in Projects. A Game-Theoretic Analysis

5o Yan Wang (UVT), The Bridge of Dreams: Towards a Method for Operational Performance Alignment in IT-enabled Service Supply Chains

2017

or Jan-Jaap Oerlemans (UL), Investigating Cybercrime

o2 Sjoerd Timmer (UU), Designing and Understanding Forensic Bayesian Networks using Argumentation

03 Daniël Harold Telgen (UU), Grid Manufacturing; A Cyber-Physical Approach with Autonomous Products and Reconfigurable Manufacturing Machines

o4 Mrunal Gawade (CWI), Multi-core Parallelism in a Column-store

o5 Mahdieh Shadi (UVA), Collaboration Behavior

o6 Damir Vandic (EUR), Intelligent Information Systems for Web Product Search 
o7 Roel Bertens (UU), Insight in Information: from Abstract to Anomaly

o8 Rob Konijn (VU), Detecting Interesting Differences:Data Mining in Health Insurance Data using Outlier Detection and Subgroup Discovery

o9 Dong Nguyen (UT), Text as Social and Cultural Data: A Computational Perspective on Variation in Text

Io Robby van Delden (UT), (Steering) Interactive Play Behavior

II Florian Kunneman (RUN), Modelling patterns of time and emotion in Twitter \#anticipointment

I2 Sander Leemans (TUE), Robust Process Mining with Guarantees

I3 Gijs Huisman (UT), Social Touch Technology - Extending the reach of social touch through haptic technology

I4 Shoshannah Tekofsky (UvT), You Are Who You Play You Are: Modelling Player Traits from Video Game Behavior

I5 Peter Berck(RUN), Memory-Based Text Correction

I6 Aleksandr Chuklin(UVA), Understanding and Modeling Users of Modern Search Engines

17 Daniel Dimov (UL), Crowdsourced Online Dispute Resolution

I8 Ridho Reinanda (UVA), Entity Associations for Search

I9 Jeroen Vuurens (UT), Proximity of Terms, Texts and Semantic Vectors in Information Retrieval

20 Mohammadbashir Sedighi (TUD), Fostering Engagement in Knowledge Sharing: The Role of Perceived Benefits, Costs and Visibility

2I Jeroen Linssen (UT), Meta Matters in Interactive Storytelling and Serious Gaming (A Play on Worlds)

22 Sara Magliacane (VU), Logics for causal inference under uncertainty

23 David Graus (UVA), Entities of Interest - Discovery in Digital Traces

24 Chang Wang (TUD), Use of Affordances for Efficient Robot Learning

25 Veruska Zamborlini (VU), Knowledge Representation for Clinical Guidelines, with applications to Multimorbidity Analysis and Literature Search

26 Merel Jung (UT), Socially intelligent robots that understand and respond to human touch

27 Michiel Joosse (UT), Investigating Positioning and Gaze Behaviors of Social Robots: People's Preferences, Perceptions and Behaviors

28 John Klein (VU), Architecture Practices for Complex Contexts 
29 Adel Alhuraibi (UvT), From IT-BusinessStrategic Alignment to Performance: A Moderated Mediation Model of Social Innovation, and Enterprise Governance of IT”

30 Wilma Latuny (UvT), The Power of Facial Expressions

3I Ben Ruijl (UL), Advances in computational methods for QFT calculations

32 Thaer Samar (RUN), Access to and Retrievability of Content in Web Archives

33 Brigit van Loggem (OU), Towards a Design Rationale for Software Documentation: A Model of Computer-Mediated Activity

34 Maren Scheffel (OU), The Evaluation Framework for Learning Analytics

35 Martine de Vos (VU), Interpreting natural science spreadsheets

36 Yuanhao Guo (UL), Shape Analysis for Phenotype Characterisation from Highthroughput Imaging

37 Alejandro Montes Garcia (TUE), WiBAF: A Within Browser Adaptation Framework that Enables Control over Privacy

38 Alex Kayal (TUD), Normative Social Applications

39 Sara Ahmadi (RUN), Exploiting properties of the human auditory system and compressive sensing methods to increase noise robustness in ASR

40 Altaf Hussain Abro (VUA), Steer your Mind: Computational Exploration of Human Control in Relation to Emotions, Desires and Social Support For applications in humanaware support systems

4I Adnan Manzoor (VUA), Minding a Healthy Lifestyle: An Exploration of Mental Processes and a Smart Environment to Provide Support for a Healthy Lifestyle

42 Elena Sokolova (RUN), Causal discovery from mixed and missing data with applications on ADHD datasets

43 Maaike de Boer (RUN), Semantic Mapping in Video Retrieval

44 Garm Lucassen (UU), Understanding User Stories - Computational Linguistics in Agile Requirements Engineering

45 Bas Testerink (UU), Decentralized Runtime Norm Enforcement

46 Jan Schneider (OU), Sensor-based Learning Support

47 Jie Yang (TUD), Crowd Knowledge Creation Acceleration

48 Angel Suarez (OU), Collaborative inquiry-based learning

2018

or Han van der $\mathrm{Aa}(\mathrm{VUA})$, Comparing and Aligning Process Representations

o2 Felix Mannhardt (TUE), Multi-perspective Process Mining 
o3 Steven Bosems (UT), Causal Models For Well-Being: Knowledge Modeling, ModelDriven Development of Context-Aware Applications, and Behavior Prediction

o4 Jordan Janeiro (TUD), Flexible Coordination Support for Diagnosis Teams in DataCentric Engineering Tasks

os Hugo Huurdeman (UVA), Supporting the Complex Dynamics of the Information Seeking Process

o6 Dan Ionita (UT), Model-Driven Information Security Risk Assessment of SocioTechnical Systems

07 Jieting Luo (UU), A formal account of opportunism in multi-agent systems

o8 Rick Smetsers (RUN), Advances in Model Learning for Software Systems

o9 Xu Xie (TUD), Data Assimilation in Discrete Event Simulations

Io Julienka Mollee (VUA), Moving forward: supporting physical activity behavior change through intelligent technology

II Mahdi Sargolzaei (UVA), Enabling Framework for Service-oriented Collaborative Networks

I2 Xixi Lu(TUE), Using behavioral context in process mining

I3 Seyed Amin Tabatabaei (VUA), Computing a Sustainable Future

I4 Bart Joosten (UVT), Detecting Social Signals with Spatiotemporal Gabor Filters

Is Naser Davarzani (UM), Biomarker discovery in heart failure

I6 Jaebok Kim (UT), Automatic recognition of engagement and emotion in a group of children

I7 Jianpeng Zhang (TUE), On Graph Sample Clustering

I8 Henriette Nakad (UL), De Notaris en Private Rechtspraak

I9 Minh Duc Pham (VUA), Emergent relational schemas for RDF

20 Manxia Liu (RUN), Time and Bayesian Networks

2I Aad Slootmaker (OUN), EMERGO: a generic platform for authoring and playing scenario-based serious games

22 Eric Fernandes de Mello Araujo (VUA), Contagious: Modeling the Spread of Behaviours, Perceptions and Emotions in Social Networks

23 Kim Schouten (EUR), Semantics-driven Aspect-Based Sentiment Analysis

24 Jered Vroon(UT), Responsive Social Positioning Behaviour for Semi-Autonomous Telepresence Robots

25 Riste Gligorov (VUA), Serious Games in Audio-Visual Collections 
26 Roelof Anne Jelle de Vries (UT),Theory-Based and Tailor-Made: Motivational Messages for Behavior Change Technology

27 Maikel Leemans (TUE), Hierarchical Process Mining for Scalable Software Analysis

28 Christian Willemse (UT), Social Touch Technologies: How they feel and how they make you feel

$29 \mathrm{Yu} \mathrm{Gu}$ (UVT), Emotion Recognition from Mandarin Speech

30 Wouter Beek, The "K" in "semantic web" stands for "knowledge": scaling semantics to the web

or Rob van Eijk (UL),Web privacy measurement in real-time bidding systems. A graph-based approach to RTB system classification

o2 Emmanuelle Beauxis Aussalet (CWI, UU), Statistics and Visualizations for Assessing Class Size Uncertainty

o3 Eduardo Gonzalez Lopez de Murillas (TUE), Process Mining on Databases: Extracting Event Data from Real Life Data Sources

04 Ridho Rahmadi (RUN), Finding stable causal structures from clinical data

o5 Sebastiaan van Zelst (TUE), Process Mining with Streaming Data

o6 Chris Dijkshoorn (VU), Nichesourcing for Improving Access to Linked Cultural Heritage Datasets

o7 Soude Fazeli (TUD), Recommender Systems in Social Learning Platforms

o8 Frits de Nijs (TUD), Resource-constrained Multi-agent Markov Decision Processes

o9 Fahimeh Alizadeh Moghaddam (UVA), Self-adaptation for energy efficiency in software systems

Io Qing Chuan Ye (EUR), Multi-objective Optimization Methods for Allocation and Prediction

II Yue Zhao (TUD), Learning Analytics Technology to Understand Learner Behavioral Engagement in MOOCs

I2 Jacqueline Heinerman (VU), Better Together

I3 Guanliang Chen (TUD), MOOC Analytics: Learner Modeling and Content Generation

I4 Daniel Davis (TUD), Large-Scale Learning Analytics: Modeling Learner Behavior \& Improving Learning Outcomes in Massive Open Online Courses

is Erwin Walraven(TUD), Planning under Uncertainty in Constrained and Partially Observable Environments

I6 Guangming Li (TUE), Process Mining based on Object-Centric Behavioral Constraint (OCBC) Models 
I7 Ali Hurriyetoglu (RUN), Extracting actionable information from microtexts

I8 Gerard Wagenaar (UU), Artefacts in Agile Team Communication

19 Vincent Koeman (TUD), Tools for Developing Cognitive Agents

20 Chide Groenouwe (UU), Fostering technically augmented human collective intelligence

2I Cong Liu (TUE), Software Data Analytics: Architectural Model Discovery and Design Pattern Detection

22 Martin van den Berg (VU),Improving IT Decisions with Enterprise Architecture

23 Qin Liu (TUD), Intelligent Control Systems: Learning, Interpreting, Verification

24 Anca Dumitrache (VU), Truth in Disagreement - Crowdsourcing Labeled Data for Natural Language Processing

25 Emiel van Miltenburg (VU), Pragmatic factors in (automatic) image description

26 Prince Singh (UT), An Integration Platform for Synchromodal Transport

27 Alessandra Antonaci(OUN), The Gamification Design Process applied to(Massive) Open Online Courses

28 Esther Kuinderman (UL), Cleared for take-off: Game-based learning to prepare airline pilots for critical situations

29 Daniel Formolo (VU), Using virtual agents for simulation and training of social skills in safety-critical circumstances

30 Vahid Yazdanpanah (UT), Multiagent Industrial Symbiosis Systems 



\section{About the Author}

Vahid Yazdanpanah is an academic researcher focused on distributed Artificial Intelligence (AI) and Multiagent Systems (MAS). In December 20I5, he started his PhD research at the Department of Industrial Engineering and Business Information Systems, University of Twente, The Netherlands. Vahid's PhD research was conducted within the context of the SHAREBOX project with the focus on developing intelligent decision support tools for industrial symbiotic relations. His work was funded by the European Union's Horizon 2020 research and innovation program. The current book is the dissertation that summarizes his research in the context of the SHAREBOX project.

In a broader perspective, Vahid's research interests include Agent-Oriented Computing, Model-Based AI, Supervisory Control Theory, Formal Methods, and Game Theory. He published his work in the International Journal on Engineering Applications of Artificial Intelligence and in proceedings of various conferences in the area of agents and multiagent systems, including the International Conference on Autonomous Agents and Multiagent Systems (AAMAS) and the European Conference on Multiagent Systems (EUMAS). Vahid received the "Best Paper Award" at the 19th International Conference on Principles and Practice of Multiagent Systems (PRIMA-2016). His professional services to the community of artificial intelligence include serving as a program committee member of the 34th International Conference of the Association for the Advancement of Artificial Intelligence (AAAI-2020) and the 24th European Conference on Artificial Intelligence (ECAI-2020). 



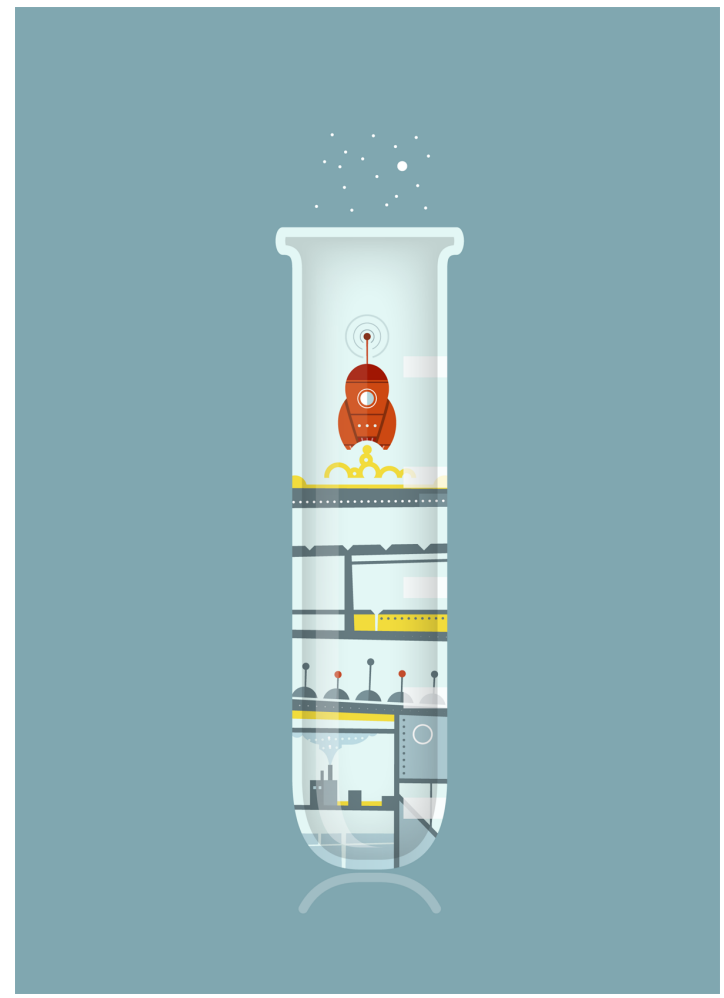

His THESIS WAS TYPESET using LTEX, originally developed by Leslie Lamport and based on Donald Knuth's TEX. The body text is set in II point Egenolff-Berner Garamond, a revival of Claude Garamont's humanist typeface. The above illustration, "Science Experiment $\mathrm{O2}^{2}$ ", was created by Ben Schlitter and released under CC BY-NC-ND 3.o. A template that can be used to format a $\mathrm{PhD}$ thesis with this look and feel has been released under the permissive MIT (XII) license, and can be found online at github.com/suchow/Dissertate or from its author, Jordan Suchow, at suchow@post.harvard.edu. 



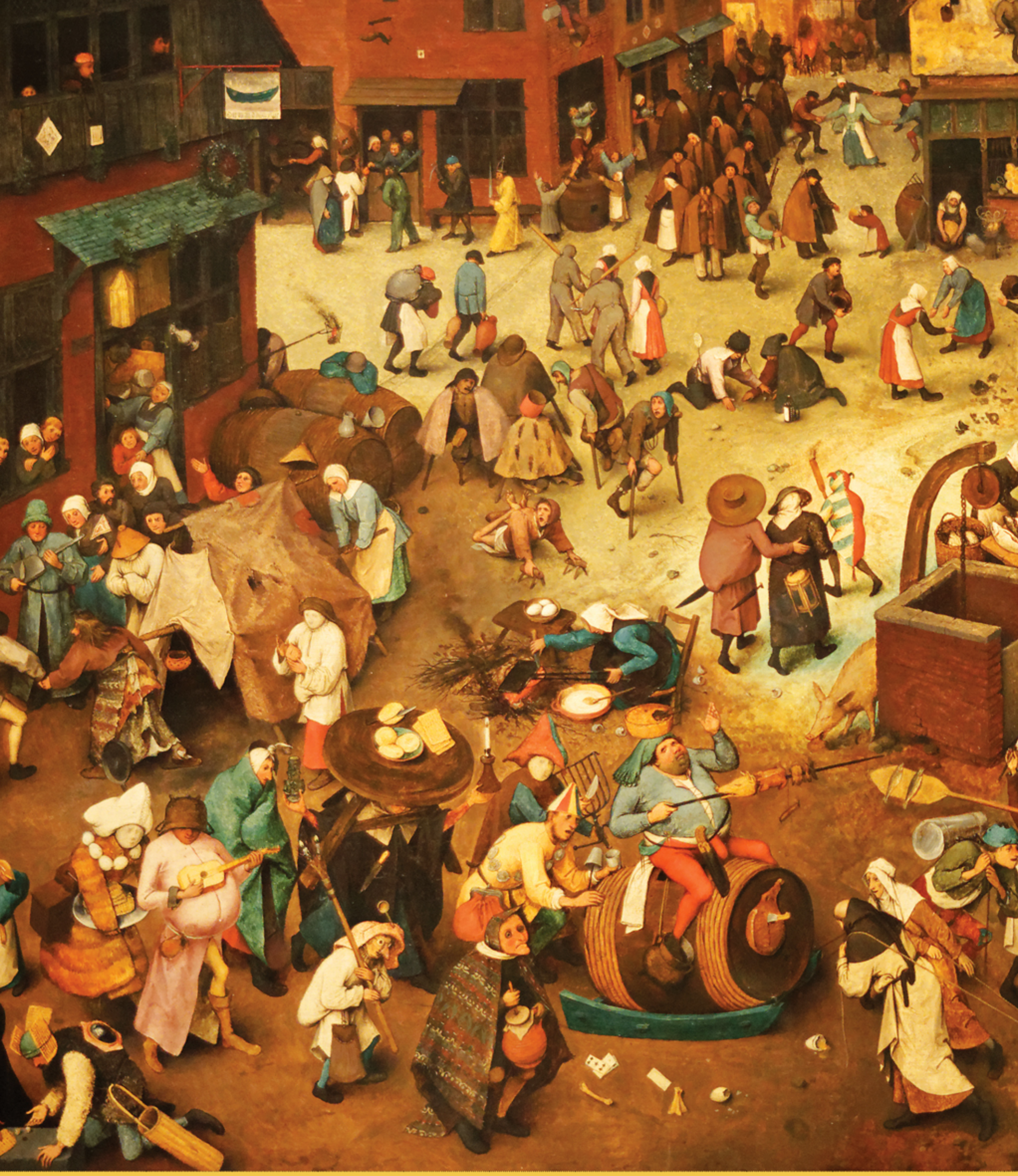

\section{UNIVERSITY OF TWENTE.}

Faculty of Behavioural, Management and Social sciences Department of Industrial Engineering and Business Information Systems
Dutch Research School for Information and Knowledge Systems The research contained in this dissertation has been funded by the European Union's Horizon 2020 research and innovation programme 